\title{
The generation of tunable coherent far-infrared radiation using atomic Rydberg states
}

\author{
William Bookless \\ (Ph.D. Thesis)

December 1980 


\title{
The generation of tunable coherent far-infrared radiation using atomic Rydberg states
}

\author{
William Bookless \\ (Ph.D. Thesis)
}

Manuscript date: December 1980

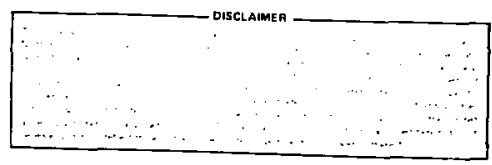

LAWRENCE LIVERMORE LABORATORY University of California $\bullet$ Livermore, California $\bullet 94550$ 
Bookless, William A., The Generation of Tunable Coherent Far-Infrared Radiation using Atomic Rydberg States, PhD, Department of Physics and Astronomy, December, 1980

A source of tunable far-infrared radiation has been constructed. The system has been operated at $91.6 \mathrm{~cm}^{-1}$ with a denonstrated tunability of $.63 \mathrm{~cm}^{-1}$. The system is based on a Rydberg state transition in optically pumped potassium vapor. The transition energy is tuned by the application of an electric field to the excited vapor. The transition wavelength and the shifted wavelength were detected and measured by the use of a Michelson interferometer an! a liquid helium cooled Ga:Ge bolometer and the data was reduced using Fast Fourier transform techniques.

Extensive spectroscopy was done on the potassium vapor to elucidate the depopulation paths and rates of the excited levels. Both theoretical and experimental results are presented to support the conclusions of the research effort. Additionally, possible alternative approaches to the population of the excited state are explored and recommendations are made for the future developinent of this source as well as the potential uses of it in molecular spectroscopy. 
THE GENERATION OF TUNABLE COHERENT FAR-INFRARED

RADIATION USING ATOMIC RYDBERG STATES

by

Willian Book less

\author{
A Thesis \\ Submitted to the \\ Department of Physics and \\ and Astronomy
}

The Graduate School of the University

of Wyoming in Partial Fulfillment of Requirements

for the Degree of

Doctor of Philosophy

University of Wyoming

Laramie, Wyoming

December, 1980 


\section{ACKNOWLEDGMENTS}

I would like to express my sincere thanks to everyone in the Chemistry Department at the Lawrence Livermore National Laboratory for making my rasearch there both very rewarding and pleasant. In particular, I would like to thank Or. L. Hrubesh and Dr. C. G. Stevens for their complete support and guidance throughout the course of the work and D. Johnson and L. L. Steinmetz for their help in many facets of the planning and building of the apparatus. I would also like to thank Helen Meredith for her excellent work and patience in typing the manuscript. Special thanks is jue to Dr. E. A. Rinehart for his guidance and for his effort in making my stay at Livermore possible.

I would like to express my appreciation to the Associated Western Universities, Inc. for their financial support throughout my research.

I wish to express special gratitude to my wife, Stacy, for her complete support during my graduate career. Her encouragement has been greatly appreciated. 
TABLE OF CONTENTS

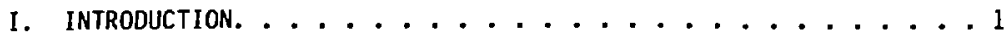

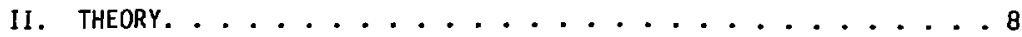

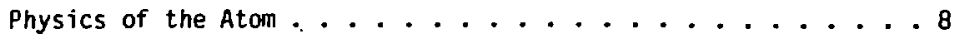
Stark Effect.......................... 30

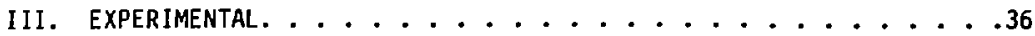

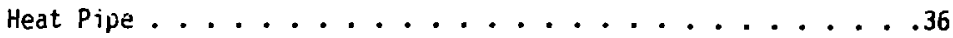

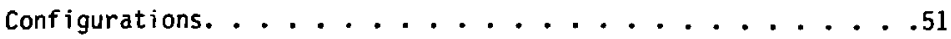
Experimental Procedure. . . . . . . . . . . . . . 59

IV. RESULTS ................................. 100

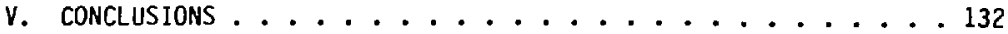


LIST OF FIGURES

Figure

Page

1. Absorptivity versus frequency for diatomic rotational

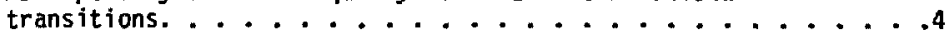

2. The Grotritn diagram for atomic potassium with some

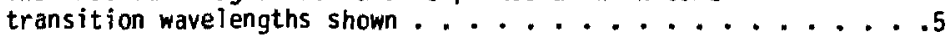

3. An expanded diagran of potassium Rydberg states with the nearest-neighbor tranșition energies shown. A? 1 levels and transition energies are in $\mathrm{cm}^{-1} \ldots \ldots . . . . . . .6$

4. A schematic diagram of a heat pipe oven ...........38

5. The temperature profile of a heat pipe containing lithium . . .41

6. A schematic diagram of the "can" type heat pipe oren with

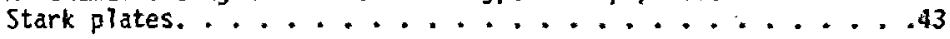

7. A photograph of the "can" type heat pipe oven with Stark

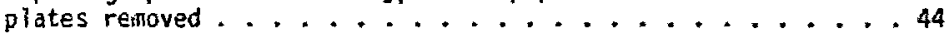

8. Sketch of the "pancake" heat pipe oven. . . . . . . . . . 47

9. Cross section view of the "pancake" heat pipe oven. . . . . 48

10. Power spectrum for R6G laser dye when used in a Molectron $\mathrm{DL}-200$ laser pumped by a Molectron UV-1000 $\mathrm{N}_{2}$ laser. . . . . 53

11. The experimental configuration used with the $\mathrm{N}_{2}$-Oye laser

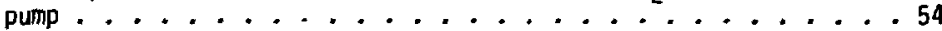

12. The experimental configuration used with the Chromatix

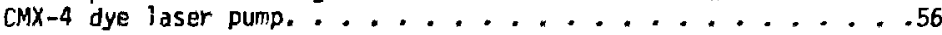

13. A typical etalon scan of the Spectra-Physics 375 dye Taser

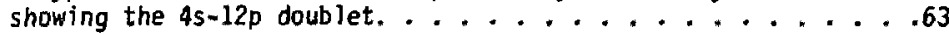

14. The configuration used for C.W. spectroscopy using the Spectra-Physics $170 \mathrm{~A}^{+}-375$ dye laser pump. .........65

15. The opto-galvanic signal associated with the group of lines

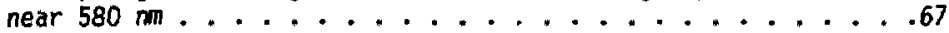

16. The configuration used for the C.W. $\mathrm{Kr}^{+}$-dye laser, CMX-4 pulsed laser experiments. . . . . .............69

17. FIR output from the detectors as the pump laser is scanned.

a) the golay cell, b) the Ga:Ge bolometer with < $2 \mu \mathrm{A}$ current,

c) the Ga:Ge bolometer with $8.2 \mu \mathrm{A}$ current. . . . . . . . 80 
18. The window function used to "taper" the data. The $50 \%$ function is a true "Hanning" function. ..........85

19. The bolometer response to a broadband source (hot wire) showing the water spectrum due to atmospheric wačer ......88

20. The schematic of the high voltage pulse generator.......90

21. A schem.ttic diagran of the total FIR experimental apparatus . . .93

22. The absorption spectrum of the U.V. highly reflecting mirror

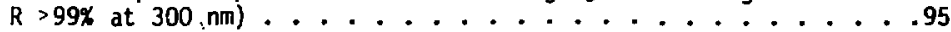

23. The absorption of the pump laser for the 4s-13p transition

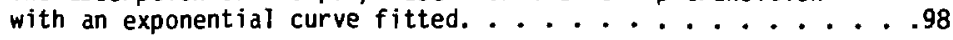

24. The fluorescence from the $K$ vapor when pumped at $767 \mathrm{~nm}$, a) on the $4 s-4 p$ transition, b) $.04 \mathrm{~nm}$ off of the $4 \mathrm{~s}-4 \mathrm{p}$ transition. ............................103

25. The diagram of the molecular potassium system showing the proposed dissociative ground state of the broad molecular

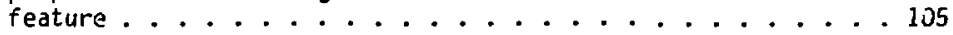

26. A scan of the fluorescence from the potassium vapor from 400-600 $\mathrm{nm}$ pumped to the 13p level. No temporal filtering

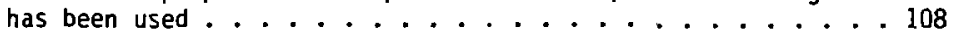

27. The same $s c$ an as Figure 26 , but with the addition of temporal filtering using a boxcar integrater, a) hot $k$ vapor, b) cold (for background signal) ......... 110,111

28. The same as 27 , but from $600-800 \mathrm{~nm}$. The line at $674 \mathrm{~nm}$ is the second grating order of scattered $337 \mathrm{~nm} \mathrm{~N} \mathrm{~N}_{2}$ laser light, a) het $K$ vapor, b) cold. ........... 113,114

29. The same as 27 , but from $400-800 \mathrm{~nm}$. The broad background about $700 \mathrm{~nm}$ is due to a fluorescing filter. Not the partial absorption of the broad fluorescence about the sharp peaks near $690 \mathrm{~nm}$, a) hot $\mathrm{K}$ vapor, b) cold. . . . . 115,116

30. Photographs of the temporal character of the groups of lines shown in Figure $27 \mathrm{a}$, a) $475.4 \mathrm{~nm}$, b) $480.2 \mathrm{~nm}$, c) $486.5 \mathrm{~nm}$, d) $495.3 \mathrm{~nm}$, e) $510.8 \mathrm{~nm}$, f) $533.2 \mathrm{~nm}$, g) $579.8 \mathrm{~nm}, \mathrm{~h}) 585.5 \mathrm{~mm}$, i) same as (h), but on a 20

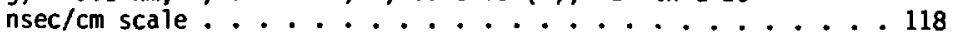

31. Typical output for a low resolution scan of the

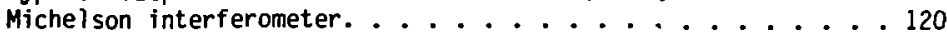


32. Fourier transform spectrum of the output of the heat pipe using the Ga:Ge bolimeter, a) total spectrum,

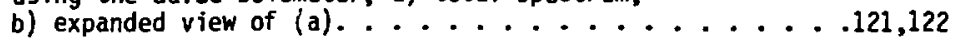

33. High resolution scan of the heat pipe output using the

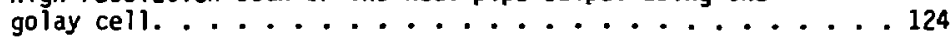

34. High resolution scan of the "pancake" heat pipe output with

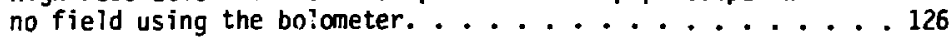

35. Output of the "pancake" heat pipe with the field on

$(-2000 \mathrm{~V} / \mathrm{cm})$ and off, a) from $0-100 \mathrm{~cm}^{-1}$, b) from

88-100 $\mathrm{cm}^{-1}$............................ 128-129

36. Expanded view of Figure 19 showing more clearly the water

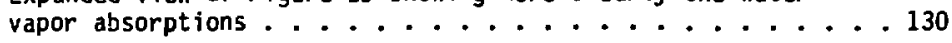


Table

1. Potassium energy levels measured and calculated. All energies

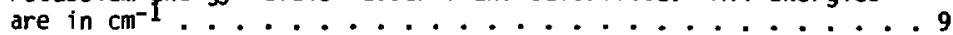

2. Transitions from $13 p \rightarrow n s$ and $13 p \rightarrow n d$ with the $A$ coefficients, lifetimes, and doppler linewidths as well as the total $A$

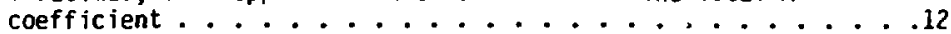

3. The quantum defects for potassium $(9<n<15)$. . . . . . . 18

4. Transitions from $11 d-n p$ and 11.d-nf with the A cuefficients and lifetimes as weli as the total A coefficient. . . . . . . .28

5. Results from the calculations by Zimmerman on the potassium

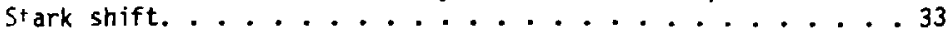

6. Potassium shifts expected for $2000 \mathrm{y} / \mathrm{cm} \ldots . . . . . . . .34$

7. The data for the absorption measurements on potassium vapor

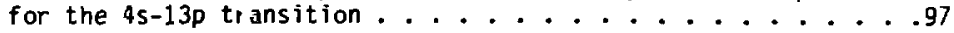




\section{CONTENTS OF APPENDICES}

Appendix

A. The computer program to take the interferometric data and do the Fast Fourier Transform (FFT) and plot the risults on an X-Y plotter with axis................ 142

B. The computer program to take and plot the fluorescence spectra. . . ....................... 149 


\section{INTRODUCTION}

The generation of tunable Far-infrared (FIR) radiation has long beer: the object of experijental stujy. In the past, the mest common sources in this region of the spectrum $(100 \mu-1000 \mu)$ have been black-body radiators. The appearance of the maser and iater the laser stimulated interest in the FIR region of the electromagnetic spectrum. This region is only now becoming accessible to the techniques of laser spectroscopy.

The earliest attempts tri generate tunable radiatisn were through the use of mixing Lechniques whereby two suitable infrared laser beams separated in frequency by the appropriate anrunt would be mixed in a crystal with a high non-linear indei. The difference frequency produced is in the FIR region.(1-9) All of these systems have suffered from either a very limited tuning range or a very large linewidth which has prevented their general acceptance as spectroscopic sources.

The most popular FIR laser source has been the optically pumped molecular laser which generally consists of a $\mathrm{CO}_{2}$ liser pumping molecules such as $\mathrm{CH}_{3} \mathrm{OH}$ to produce very strong, narrow laser lines in the FîR region. These systems are, however, only line tunable; attempts to Starix tune then to frnduce a coritinuously tunable source have produced tuning rariges of only about 10 $\mathrm{MHz}$ (10). An excellent revies of the progress in source development is given in ( $\left.{ }^{\prime}\right)$.

The method proposed here is based on the very small separation between the Rydberg states atomic alkali metals. When one reaches states with the princifle quantum number $r$. greater than 11 
the energy separation between adjacent states with allowed dipole transitions is in the FIR region. These levels have also shown enormous $S^{*}$ ark polarizabilities (12) which would make the transitions putentially tunabie over a wide range. The linewidths of these transitions are limited fundamentally by the decay rates of the: states which in the absence of collisions would be only 180 $\mathrm{KH} \%$, but prartically are limited to about $10 \mathrm{MHz}$. The large number of FIR transitions available in the alkali metals coupled with the large tunabilities available make this method of generation a very attractive and potentially quite useful source.

The upper states of the alkali metals have long been known to display stimulated emission and have been studied using laser excitation for over 13 years (13). More recently, lasing has been shown in alkali metal systems $(14,15)$. These methods were extended in a proposal type paper by Lau et al. (16) to include the possibility of Stark tuning the transitions. In (17), optically pump stimulated emission was shown to occur in the FIR region from the highly excited Rydberg states in potassium. This work has been the experimental basis for the work presented here. A very interesting and informative article on the experimental work on the Rydberg states of atoms has been recently published by Latimer (18) and gives an excellent background for our work.

The tunability of this system as mentioned above, comes from the large Stark polarizabilities of the atoms and thus requires an applied eiectric field for its implementation. This field can be in the form of a periodic field giving the dynamic Stark effect, 
or in the form of a d.c. field for the d.c. Stark effect. The former has been demonstrated using a pulsed $\mathrm{CO}_{2}$ laser, but the equipment requirements are prohibitive for many applications. The latter method has been demonstrated in an atomic beam apparatus (19). This metnod was limited in output power by the extremely small number of atoms available in an atomic beam.

This research grew out of the need for a highly sensitive monitor for gases. In the past the microwave region of the spectrum has been the most selective area to do the work, but has suffered from a lack of sensitivity. This problem could be illeviated by the use of FIR radiation to do pure rotational spectroscopy on the molecular systems of inierest. As shown in Figure (1) this region of the spectrum offers an increase in absorptivity of up to $10^{3}$ over similar processes in the microwave region. The author has been interested in the application of lasers to atomic and molecular physics and this project provided a vehicle by which all of these interests could be satisfied.

To begin, the particular system of interest is described. A source if FIR radiation based on the $13 p-11 d$ transition in atonic potassium has been modeled and constructed. The Grotrian diagram in Figure (2) shows the energy levels of the potassium atom and the transition energies between the ground state and the excited states connected $i y$ dipole transitions. The transitions of interest are shown in more detail in Figure (3).

In this thesis the theoretical and experimental work done to demonstrate the generation of tunable FIR radiation in the region 


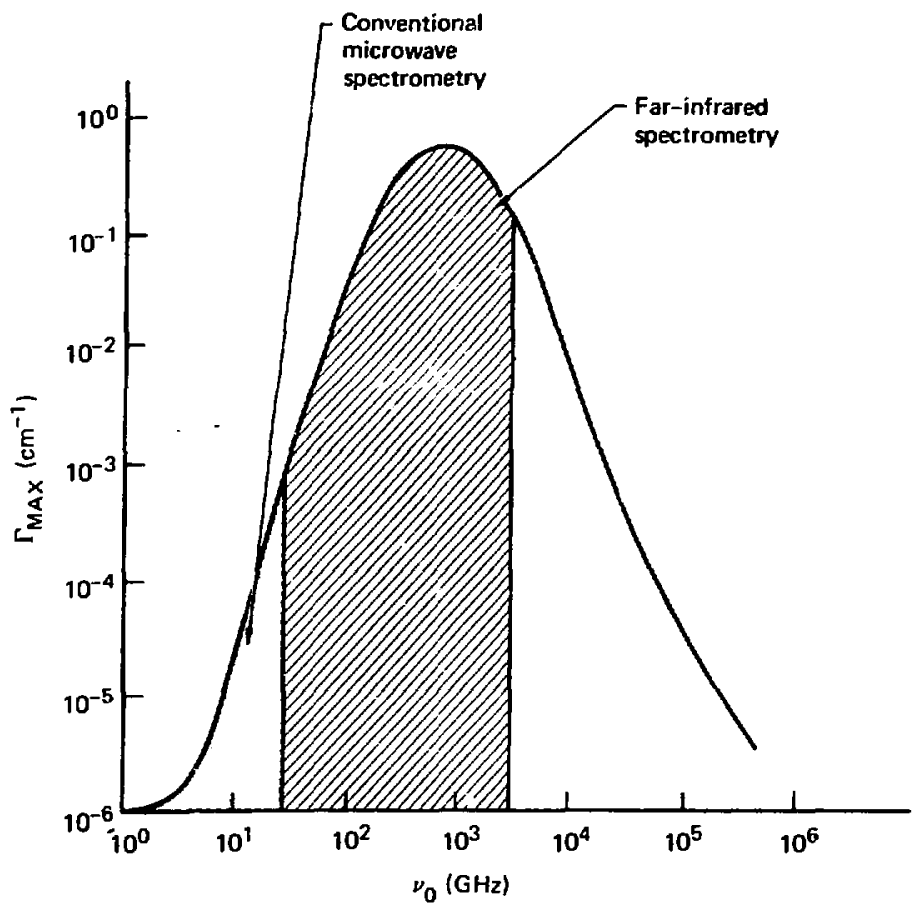

Figure 1. Absorptivity versus frequency for diatomic rotational transitions. (58) 


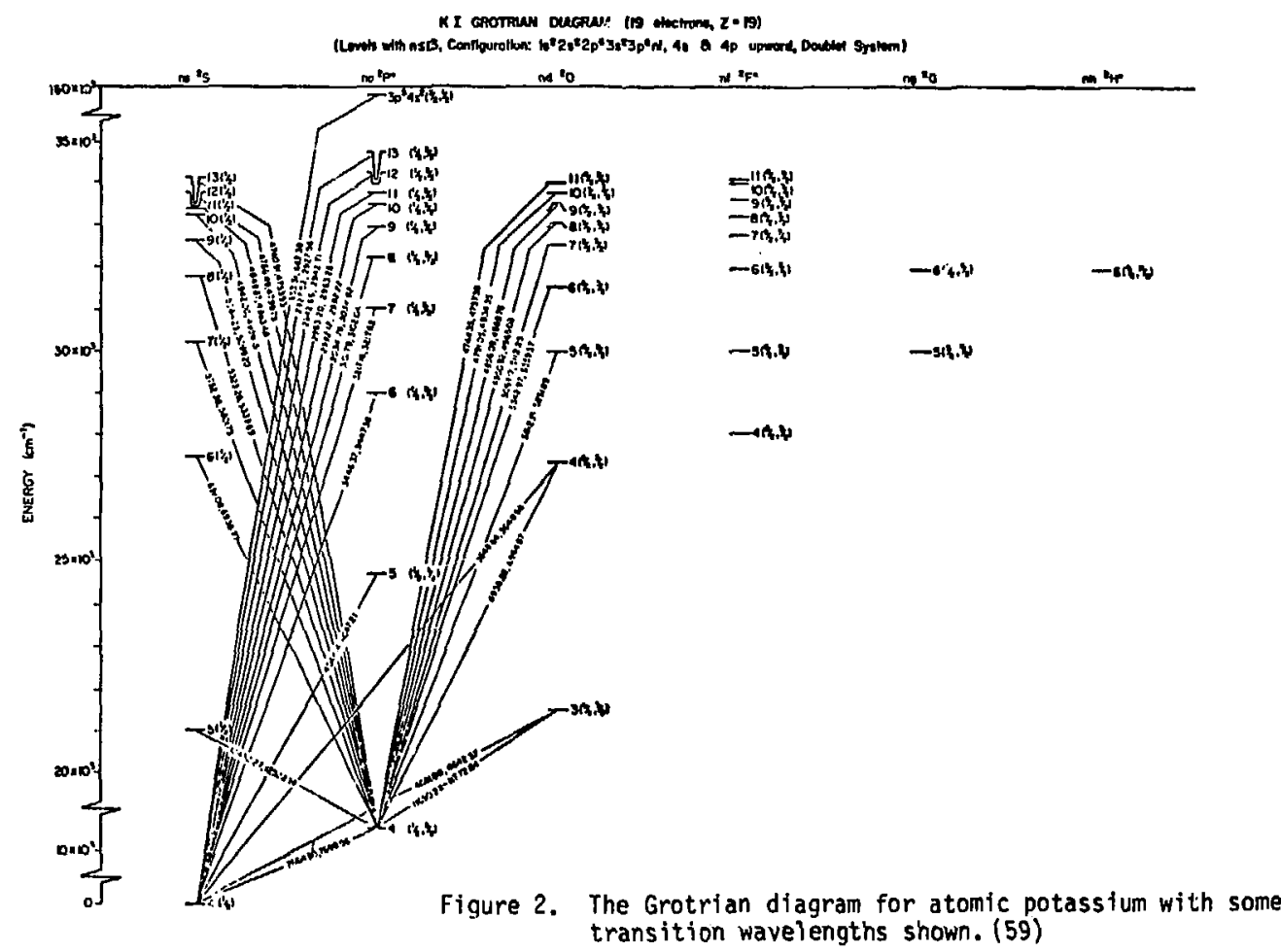




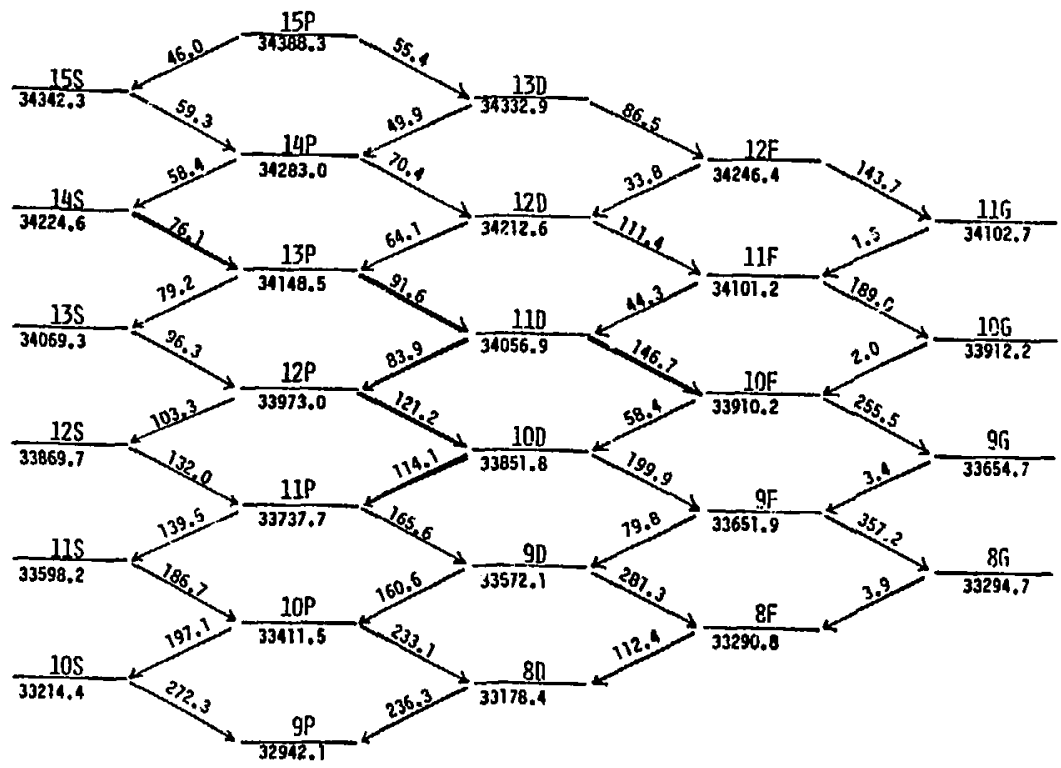

Figuit 3. An expanded diagram of potassium Rydberg states with the nearest-neighbor transition energies shown. All levels and transition energies are in $\mathrm{cm}^{-1}$. 
of $92 \mathrm{~cm}^{-1}(109 \mu \mathrm{M})$ will be outlined. The next chapter will concentrate on the theory of highly excited atoms and their depopulation from upper excited states by radiative and nonradiative (collisional) processes. A discussion of the Stark effect will be included. The third chapter will discuss the experimental arrangenents used and the configurations considered. The fourth chapter will examine the results of these experiments and our conclusions will be presented in the final chapter. 


\section{THEORY}

The method used here for the generation of tunable submillimeter radiation can be addressed thenretically in a very straightforward fashion. We can break the theoretical treatment up into two general areas and investigate each independently.

\section{Physics of the Atom}

The first area to be considered is that of the atomic physics of a highly excited atom. In particular, since an alkali metal is being considered, many simplifying approximations can be made for the quantum mechanics of the atom. Core interactions are going to become Jecreasingly important the further from the core and in fact $c$ an be ignored when high $n$ states are dealt with. Table 1 shows that at high $n$ values and large 1 values, a simple Rydberg formula using effective quantum numbers supplied by experinent, describes the energy levels of the atom quite well. The measured values for some of these levels is also given for comparison at lower values.

Similarly, at high values of $n+1$ the semi-empirical methods of Bates and Damgaard (20) provide accurate results for the transition probabilities. A semi-emperical method used by Anderson and Zilitis (21) to calculate the oscillator strengths of potassium for all of the transitions of interest will be discussed. From these oscillator strengths the transition probabilities and natural lifetimes of states will then be calculated. Using these lifetimes and probabilities the absorption cross sections as well as the stimulated emission and spontarieous cross sections for the 
TABLE 1. Potassium energy levels measured and calculated. A1l energies are in $\mathrm{cm}^{-1}$

\begin{tabular}{|c|c|c|c|c|c|}
\hline Level & Moore $(\mathrm{J})$ & Calculated & Leve] & Moore $(\mathrm{J})$ & Calculated \\
\hline $\begin{array}{r}4 s \\
4 p \\
5 s \\
3 d \\
5 p \\
4 d \\
6 s \\
4 f \\
6 p \\
5 d \\
7 s \\
5 f \\
5 g \\
7 p \\
6 d \\
8 s \\
6 f \\
6 g \\
8 p \\
7 d \\
9 s \\
7 f \\
7 g \\
9 p \\
8 d \\
10 s\end{array}$ & $\begin{array}{l}0 \\
13042.89(3 / 2) \\
21016.80(3 / 2) \\
21536.75(3 / 2) \\
24720.20(3 / 2) \\
27398.11 \quad(3 / 2) \\
27450.65 \\
28127.70 \\
29007.70(3 / 2) \\
30185.69(3 / 2) \\
30274.26 \\
30605.60 \\
30619.80 \\
31074.46(3 / 2) \\
31695.75(3 / 2) \\
31764.95 \\
31953.00 \\
31960.8 \\
32230.12(3 / 2) \\
32598.46 \\
32648.17 \\
32764.52 \\
32942.08(3 / 2) \\
33178.36 \\
33214.39\end{array}$ & $\begin{array}{l}14046.80 \\
21229.72 \\
20317.62 \\
24858.96 \\
27134.81 \\
27497.30 \\
28116.61 \\
29041.41 \\
30110.95 \\
30290.12 \\
30602.57 \\
30618.42 \\
31085.30 \\
31670.90 \\
31772.20 \\
31951.26 \\
31960.43 \\
32234.29 \\
32589.04 \\
32651.79 \\
32763.78 \\
32769.55 \\
32943.69 \\
33174.64 \\
33216.16\end{array}$ & $\begin{array}{r}8 f \\
8 g \\
10 p \\
9 d \\
11 s \\
9 f \\
9 g \\
11 p \\
10 d \\
12 s \\
10 f \\
10 g \\
12 p \\
11 d \\
13 s \\
11 f \\
11 g \\
13 p \\
12 d \\
14 s \\
12 f \\
14 p \\
13 d \\
15 s \\
15 p\end{array}$ & $\begin{array}{l}33411.54(3 / 2) \\
33572.11 \\
33598.17 \\
3 \vdots 652.0 \\
33737.44(3 / 2) \\
33851.76 \\
33869.7 \\
\\
34148.63(3 / 2) \\
34056.7 \\
34069.3 \\
34148.63(3 / 2) \\
\\
34283.15(3 / 2) \\
34388.46(3 / 2)\end{array}$ & $\begin{array}{l}33290.80 \\
33294.66 \\
33412.19 \\
33570.86 \\
33599.74 \\
33651.95 \\
33654.66 \\
337.37 .68 \\
33851.34 \\
33872.24 \\
33910.18 \\
33912.16 \\
33972.96 \\
34057.15 \\
34072.76 \\
34101.18 \\
34102.67 \\
34148.53 \\
34212.60 \\
34224.58 \\
34246.42 \\
34283.00 \\
4332.92 \\
34342.28 \\
34388.27\end{array}$ \\
\hline
\end{tabular}


FIR transitions of interest. He will also examine the competing processes to the stimulated enission and compare these rates to those of the desired transitions. Finally we will look at the conditions for lasing in the $K$ vapor and discuss the threshold conditions for super fluorescence as well as tiue lasing.

We begin by looking at the methods used for the calculation of the oscillator strencths of potassium. If we designate $P(r)$ as the radial part of the wave function of the outer or valence electron then we can write

$$
\psi(r, \theta, \phi)=P(r) / r \quad Y(\theta, \phi)
$$

where $Y(\theta, \phi)$ are the well known spherical harmonic functions. The oscillator strengths can be calculated from the matrix elements

$$
\left(r_{i k}\right)=\int_{0}^{\infty} P_{k} r P_{i} d r .
$$

If we are interested only in the average oscillator strength, then we can write

$$
f_{i k}=\frac{\max \left(l_{i} l_{k}\right)}{2 T_{i}+1} \frac{2}{3}\left(E_{k}-E_{i}\right)\left(r_{i k}\right)^{2}
$$

where $\max \left(l_{i}, l_{k}\right)$ is the maximum az imuthat quant im number $l_{j}$ or $l_{k}$ of the initial and final stites. $E_{j}$ and $E_{k}$ are the initial and final energies in atomic units (21). This calculation does not take into consideration the sign of $f_{i k^{*}}$ The functions $P(r)$ have been calculated by the semi-emperical system proposed in (22). Equation (3) is solved for $P(r)$, where $E_{\exp }$ is the 


$$
\ddot{p}(r)+2 E_{\exp }+\frac{2 z}{r}-2 V(r)-\frac{\ell(\ell+1)}{r 2} P(r)=0
$$

experimental value of the ionization potential for potassium and $V(r)$ is the screening potential taken from the self-consistent field method of (23).

These calculations have been carried out by Anderson and zilitis (a1) and they are used without further improvement. As the high ri-value states of the potassil'n atom have a very large radius, $r$ goes as $n^{2}$, we expect the approximations of Anderson and zilitis to be quite good in the region of $n=13$.

Using these oscillator strengths we can calculate the spontaneous transition probabilities by using

$$
A_{i k}=2 \alpha^{3}\left(E_{k}-E_{i}\right)^{2} f_{i k}
$$

or in units of $\sec ^{-1}$

$$
A_{i k}=0.6669\left(E_{k}-E_{i}\right)^{2} f_{i k}
$$

where $E$ is measured in $\mathrm{cm}^{-1}$.

This is the probability of a transition from level $i$ to level $k$ in $\sec ^{-1}$ by spontaneous decay. The spontaneous lifetine of a level is then

$$
\tau_{i}=\frac{1}{A_{i \tau}}=\left[\begin{array}{ll}
\sum_{k} & A_{i k}
\end{array}\right]^{-1}
$$

An example of this is in Table (2). This illustrates the case for spontaneous decay from the $13 \mathrm{P}$ state of potassium in the absence ef collisions. 
TABLE 2. Transitions from $13 p \rightarrow n s$ and $13 p \rightarrow n d$ with the $A$ coefficients, lifetime, and doppler linewidths as well as the total A coefficient.

\begin{tabular}{|c|c|c|c|c|c|c|}
\hline Transition & $f_{i k}$ & $\left(E_{k}-E_{i}\right) \mathrm{cm}^{-1}$ & $A_{i k} \sec ^{-1}$ & $t_{i k} \sec$ & $v \sec ^{-1}$ & $\Lambda \nu_{d} \sec ^{-1}$ \\
\hline $\begin{array}{rr}13 p & 13 s \\
13 p & 12 s \\
13 p & 11 s \\
13 p & 10 s \\
13 p & 9 s \\
13 p & 8 s \\
13 p & 7 s \\
13 p & 6 s \\
13 p & 5 s \\
13 p & 4 s\end{array}$ & $\begin{array}{l}1 / 3 \times 4.87 \\
1 / 3 \times .170 \\
1 / 3 \times .0341 \\
1 / 3 \times .0115 \\
1 / 3 \times .00487 \\
1 / 3 \times .00225 \\
1 / 3 \times .00101 \\
1 / 3 \times .000354 \\
1 / 3 \times .000170 \\
1 / 3 \times .0000560\end{array}$ & $\begin{array}{l}7.933 \times 10^{1} \\
2.79 \times 10^{2} \\
5.50 \times 10^{2} \\
9.34 \times 10^{2} \\
1.50 \times 10^{3} \\
2.38 \times 10^{3} \\
3.87 \times 10^{3} \\
6.70 \times 10^{3} \\
1.31 \times 10^{4} \\
3.41 \times 10^{4}\end{array}$ & $\begin{array}{l}6.81 \times 10^{3} \\
2.94 \times 10^{3} \\
2.30 \times 10^{3} \\
2.23 \times 10^{3} \\
2.44 \times 10^{3} \\
2.84 \times 10^{3} \\
3.37 \times 10^{3} \\
3.53 \times 10^{3} \\
6.51 \times 10^{3} \\
1.45 \times 10^{4}\end{array}$ & $\begin{array}{l}1.47 \times 10^{-4} \\
3.40 \times 10^{-4} \\
4.35 \times 10^{-4} \\
4.48 \times 10^{-4} \\
4.10 \times 10^{-4} \\
3.52 \times 10^{-4} \\
2.97 \times 10^{-4} \\
2.83 \times 10^{-4} \\
1.54 \times 10^{-4} \\
6.89 \times 10^{-5}\end{array}$ & $\begin{array}{l}2.38 \times 10^{12} \\
8.36 \times 10^{12} \\
1.65 \times 10^{13} \\
2.80 \times 10^{13} \\
4.50 \times 10^{13} \\
7.18 \times 10^{13} \\
1.16 \times 10^{14} \\
2.01 \times 10^{14} \\
3.94 \times 10^{14} \\
1.02 \times 10^{15}\end{array}$ & $\begin{array}{l}6.73 \times 10^{6} \\
2.37 \times 10^{7} \\
4.67 \times 10^{7} \\
7.94 \times 10^{7} \\
1.27 \times 10^{8} \\
2.03 \times 10^{8} \\
3.29 \times 10^{8} \\
5.69 \times 10^{8} \\
1.11 \times 10^{9} \\
2.90 \times 10^{9}\end{array}$ \\
\hline $\begin{array}{rr}13 p & 11 d \\
13 p & 10 d \\
13 p & 9 d \\
13 p & 8 d \\
13 p & 7 d \\
13 p & 6 d \\
13 p & 5 d \\
13 p & 4 d \\
13 p & 3 d\end{array}$ & $\begin{array}{l}2.29 \\
.163 \\
.0451 \\
.0181 \\
.00849 \\
.00417 \\
.00196 \\
.000763 \\
.000160\end{array}$ & $\begin{array}{l}9.16 \times 10^{1} \\
2.97 \times 10^{2} \\
5.76 \times 10^{2} \\
9.70 \times 10^{2} \\
1.55 \times 10^{3} \\
2.45 \times 10^{3} \\
3.96 \times 10^{3} \\
6.75 \times 10^{3} \\
1.26 \times 10^{4}\end{array}$ & $\begin{array}{l}1.28 \times 10^{4} \\
9.58 \times 10^{3} \\
1.00 \times 10^{4} \\
1.14 \times 10^{4} \\
1.36 \times 10^{4} \\
1.67 \times 10^{4} \\
2.05 \times 10^{4} \\
2.32 \times 10^{4} \\
1.70 \times 10^{4}\end{array}$ & $\begin{array}{l}7.78 \times 10^{-5} \\
1.04 \times 10^{-4} \\
1.00 \times 10^{-4} \\
8.80 \times 10^{-5} \\
7.35 \times 10^{-5} \\
5.98 \times 10^{-5} \\
4.87 \times 10^{-5} \\
4.31 \times 10^{-5} \\
5.89 \times 10^{-5}\end{array}$ & $\begin{array}{l}2.75 \times 10^{12} \\
8.90 \times 10^{12} \\
1.73 \times 10^{13} \\
2.91 \times 10^{13} \\
4.65 \times 10^{13} \\
7.36 \times 10^{13} \\
1.19 \times 10^{14} \\
2.02 \times 10^{14} \\
3.78 \times 10^{14}\end{array}$ & $\begin{array}{l}7.78 \times 10^{6} \\
1.52 \times 10^{7} \\
4.90 \times 10^{7} \\
8.24 \times 10^{7} \\
1.32 \times 10^{8} \\
2.08 \times 10^{8} \\
3.37 \times 10^{8} \\
5.74 \times 10^{8} \\
1.07 \times 10^{9}\end{array}$ \\
\hline
\end{tabular}


From this we can calculate the 8 coefficient for stimulated absorption and emission.

The stimulated emission or absorption rate $W$, and the $A$ and $B$ coefficients are related by the well known relation

$$
W=I_{\omega} B=A \frac{4 \pi^{3} C^{2}}{\hbar \omega^{3}} I \omega
$$

where $w$ is the radial frequency of stimulated transition and I is tho intensity of the radiation field per unit frequency in units of energy $x$ dis. ${ }^{-2}$. Relating the variable $\omega$ to $y$ by the reiation,

$$
\nu=\frac{\omega}{? \pi}
$$

we have,

$$
B=A \frac{4 \pi^{3} c^{2}}{h(2 \pi \nu)^{3}}
$$

or

$$
B=A \frac{c^{2}}{2 n \nu^{3}}
$$

$$
\text { for } \begin{aligned}
\hbar & =1.0547 \times 10^{-34} \mathrm{~J}-\mathrm{sec} \\
C & =2.9979 \times 10^{10} \mathrm{~cm} \mathrm{sec}^{-1} \\
\Rightarrow B & =4.2606 \times 10^{54} \frac{A}{\nu 3} \mathrm{~cm}^{2} \mathrm{~J}^{-1} \mathrm{sec}^{-1}
\end{aligned}
$$

We can find the cross section for stimulated absorption from the relation 


$$
\sigma_{\omega^{a}}=\frac{1}{4} \lambda^{2} \frac{4}{9}, a_{\omega}
$$

where $g$ and $g$ ' are the multiplicities of the beginning and final states and $a$ is ihe transition probability between $\omega$ and $\omega+d \omega$ defined by,

$$
A=\int a_{\omega} d_{\omega}
$$

From this we can calculate an integrated cross section by,

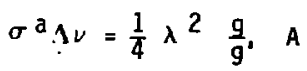

To find the average stimulated absorption cross section we must then divide by the linewidth of the spectral line, $1 v$.

To get $\sigma^{S T}$, the cross section for stimulated emission, we must multiply by the ratio of the multiplicities of the levels involved. From this we get,

$$
\sigma^{\mathrm{ST}}=\frac{\mathrm{g}^{\prime}}{\mathrm{g}} \sigma^{\mathrm{a}}
$$

or more directly in terms of $A$,

$$
\sigma^{S T}=\frac{1}{4.1 \nu} \lambda^{2} \quad A
$$

The three things affecting the linewidth $A_{t}$ of the transition are; the natural linewidth of the transition, the doppler iroac ning due to the thermal energy of the system, and the colitision broadening due to the collision between excited atoms. As will be seen, the collisions between excited atoms are the only collisions needed because of the enormous difference in physical size between excited and ground state atoms. 
From Table (2) it is seen that the natural lifetime of the potassium 13P state is approximately $5.5 \mu \mathrm{sec}$. By using the relationship,

$$
\Lambda \nu=\Lambda \omega / 2 \pi=\frac{1}{2 \pi T}
$$

the Iatural linewidth for this state is,

$$
\Delta v \simeq 2.9 \times 10^{4} \mathrm{~Hz}
$$

This is exceedingly narrow and as will be seen, is overwhelmed $b_{4}$ the other broadening mechanisms.

Next we look at the doppler broa ening of the transition due to the thermal energy of the atoms. The linewidth due to this mechanism may be written as,

$$
\begin{aligned}
1 v_{d} & =2 v_{0} \sqrt{\frac{2 k T}{M C^{2}} \ln 2} \\
& =7.162 \times 10^{-7}\left(\frac{T}{M}\right)^{1 / 2} v_{0}
\end{aligned}
$$

where $M$ is the atomic weight of the atom, $T$ is the temperature of the system in ${ }^{0_{K}}$, and $\nu_{0}$ is the center frequency or the transition. For potassium with $M=39.1$ we save,

$$
\Delta \nu_{d}=1.14 \times 10^{-7} T^{1 / 2} \nu_{0}
$$

If we again look at the $13 p$ state as the one of interest and take $\nu_{0}$ for the $45-13 p$ absorption transition we have,

$$
\nu_{0}=1.02 \times 10^{15} \mathrm{~Hz}
$$

Also, using a pressure of 1 Torr for the potassium vipor the 
temperature of the vapor from ref. (24), is

$$
T=\frac{4430}{7.1830-\log P}
$$

or $\quad T=617^{\circ} \mathrm{K}$

Using this result and equation (21) in (20) we get,

$$
\Delta \nu_{\mathrm{d}}=2.89 \times 10^{9} \mathrm{~Hz}
$$

We should note here that since this is a function of $T^{1 / 2}$, it will not vary greatly with temperature (or pressi: c).

The third broadening method to be discussed is that due to collisions. We can write the linewidth contribution due to collisions as,

$$
\Delta \nu_{c}=\omega_{c} / 2 \pi
$$

where $\omega_{c}$ is the collision frequency which in turn can be written as

$$
\omega_{c}=\sigma_{c} n v
$$

In this equation $\sigma_{c}$ is the collision cross section, $n$ is the number density of colliding atoms, and $v$ is the average velocity of the atoms. The geonetric cross section of the atom may be written as,

$$
c_{c}=\pi r_{a v}^{2}
$$

where from Ref. (25),

$$
r_{\mathrm{av}}=1 / 2\left[3 n^{\star 2}-1(1+1)\right] \mathrm{a}_{0}
$$

and $n^{*}$ is the effective quantum number defined by

$$
n^{*}=n-\mu
$$


where $\mu$ is the quantum defect, and $a_{0}$ is the Bohr radius. The quantum defects for potassium are shown in Table (3). Again using the $13 p$ state as an example,

$$
\sigma_{c}=3.46 \times 10^{-13} \mathrm{~cm}^{2}
$$

The number ciensity of excited atoms is, in the limit of saturation of the excited state, just one half of the total number density of atoms. This number density may be written,

$$
\begin{aligned}
n_{t} & =\frac{p}{k T} \\
& =\frac{1}{1.38 \times 10^{-16} \operatorname{erg}^{0}}{ }^{-1} \frac{p}{T} \\
& =7.24 \times 10^{15} \frac{\mathrm{p}}{\mathrm{T}}\left(p=\text { dynes } \mathrm{cm}^{-2}, T={ }^{0}\right)
\end{aligned}
$$

But we can write,

$$
\begin{aligned}
p(\text { Torr })=1.33 \times 10^{3} \mathrm{p} \text { (dynes } \mathrm{cm}^{-2} \text { ) } \\
\Rightarrow n_{t}=7.24 \times 10^{15} \times 1.33 \times 10^{3} \frac{\mathrm{p}}{\mathrm{T}} \text { Atoms } \mathrm{cm}^{-3} \\
=9.63 \times 10^{18} \frac{\mathrm{p}}{\mathrm{T}} \text { Atoms } / \mathrm{cm}^{-3}\left(\mathrm{p}=\text { Torr }, T={ }^{0} \mathrm{~K}\right. \text { ) }
\end{aligned}
$$

Finally we can calculate the rms velocity of the atoms from,

$$
\begin{aligned}
v & =\frac{\sqrt{3 \mathrm{kT}}}{\mathrm{m}} \\
& =2.52 \times 10^{3} \mathrm{~T}^{1 / 2} \mathrm{~cm} / \mathrm{sec}\left(T=0_{\mathrm{K}}\right)
\end{aligned}
$$

for atoms of $39.1 \mathrm{gm} / \mathrm{mole}$.

We, therefore, have combining (29), (30), and (31), 
TABLE 3. The quantum defects for potassium $(9<n<15)$

\begin{tabular}{ccc}
$\ell$ & $\mu$ & Reference \\
\hline 0 & $2.178(6)$ & $(9)$ \\
1 & $1.712(2)$ & $(9)$ \\
2 & $0.267(1)$ & $(9)$ \\
3 & $0.010(1)$ & $(9)$
\end{tabular}




$$
\begin{aligned}
\omega_{c} & =\sigma_{c} \frac{n t}{2} v \\
& =4.20 \times 10^{9} \frac{\mathrm{p}}{T^{1 / 2}} \text { radians } \mathrm{sec}^{-1}
\end{aligned}
$$

or from eq. $\{24\}$,

$$
\Delta \nu_{c}=6.68 \times 10^{8} \cdot \frac{T^{1 / 2}}{p z}
$$

For our standard case of $p=1$ torr and $T=617^{\circ} \mathrm{K}$ we have,

$$
\Delta \nu_{c}=2.69 \times 10^{7} \mathrm{sec}^{-1}
$$

We see from this result, eq. (18), and eq. (23), that line broadening for the $n=13$ state in potassium at 1 Torr and $617^{\circ} \mathrm{K}$ is essentially all due to the doppler effect. We will use only this term in calculating the average cross section from eq. (14). Using eq. (14) and the result in eq. (23) we have,

$$
\sigma^{\mathrm{a}}=\frac{1}{4} \lambda^{2} \quad \frac{\mathrm{g}}{\mathrm{g}^{\prime}} \mathrm{A} \frac{1}{2.89 \times 10^{9}}
$$

If we rewrite eq. (5) as,

$$
A_{i k}=0.6669 \frac{1}{\lambda^{2} i k} f_{i k}
$$

and combine this with the oscillator strength $f_{i k}$ from ref while noting that the multiplicities have been included in the calculations of ref (21), then we have for eq. (34),

$$
\begin{aligned}
\sigma^{\mathrm{a}} & =\frac{1}{4} \times 0.6669 \times 5.60 \times 10^{-5} \times \frac{1}{2.89 \times 10^{9}} \\
& =3.23 \times 10^{-15} \mathrm{~cm}^{2}
\end{aligned}
$$


or using eg. (15) and the multiplicities $g$ and $g^{\prime}$ for the 13p and 45 states respectively, we have for the stimulated emission cross section for the $13 p \rightarrow 4 s$ transition,

$$
\begin{aligned}
\sigma^{\mathrm{ST}} & =\frac{6}{2} \times 3.23 \times 10^{-15} \mathrm{~cm}^{2} \\
& =9.69 \times 10^{-15} \mathrm{~cm}^{2}
\end{aligned}
$$

We would now like to examine the cross sections of the other transitions out of the $13 \mathrm{p}$ excited state. To do this we must calculate the linewidths of these lines. We note here that the collisional linewidths for these lines are unchanged and will still be much larger than the very narrow natural linewidths. The doppler linewidths, however, are directly proportional to the frequency of the transition $\nu_{0}$ and will, therefore, decrease significantiy as we reduce the frequency of the transitions. The linewidths of the transitions from the 13p-nd and 13-ns states are shown in Table (2) and have been calculated using eq. (20) at a temperature of $617^{\circ} \mathrm{K}$. We see from this that for the $13 \mathrm{p}-11 \mathrm{~d}$ and the 13p-13s transitions, we can consider the collision linewidth as the dominant factor in the cross-section calculations. Therefore, for the FIR transition at $91.6 \mu \mathrm{m}$ between the $13 \mathrm{p}$ and 11d states, we have,

$$
\Delta v=\Delta v_{\mathrm{c}}=2.69 \times 10^{7} \mathrm{sec}^{-1}
$$

To be more correct we can get a first order estimate of the real linewidth by adding the collisional and doppler contributions together to get an upper limit. We then would have, 


$$
\Delta \nu=\Delta v_{\mathrm{c}}+\Delta \nu_{\mathrm{d}}=3.47 \times 10^{7} \mathrm{~Hz}
$$

We will use this in all further calculations concerning the 13p-11d transition.

Using eq. (39) and eq. (16) we can write,

$$
\sigma S T=\frac{1}{4 \times 3.47 \times 10^{7}} \lambda^{2} A
$$

and using eq. (35) we have,

$$
\begin{aligned}
\sigma^{S T} & =\frac{1}{1.39 \times 10^{8}} \times .6669 \mathrm{f}_{i k} \\
& =4.81 \times 10^{-9} \mathrm{f}_{i k}
\end{aligned}
$$

from Table (2) we have,

$$
\begin{aligned}
& f_{i k}(13 p-11 d)=2.29 \\
\Rightarrow & \sigma S T=1.10 \times 10^{-8}
\end{aligned}
$$

and lising (11) we calculate the Einstein B coefficient for this transition,

$$
\begin{aligned}
B & =4.26 \times 10^{54} \frac{A}{\nu_{i k}^{3}} \\
& =4.26 \times 10^{54} \lambda_{i k} 3 A \frac{1}{\mathrm{C}^{3}} \\
& =4.26 \times 10^{54} \times .6669 \times \lambda_{i k}{ }_{i k} \frac{1}{\mathrm{C}^{3}} \\
& =2.63 \times 10^{21} \mathrm{~cm}^{2} \mathrm{~J}^{-1} \mathrm{sec}^{-1}
\end{aligned}
$$

If we assume some intensity for the blackbody background such as, 


$$
\begin{aligned}
I= & \rho(\nu) \Delta \nu C=\frac{8 \pi h \nu^{3}}{c^{2}} \frac{1}{\exp (h \nu / k T)-1} \Delta \nu \\
= & \frac{8 \pi \times 6.63 \times 10^{-34} \times\left(2.75 \times 10^{12}\right)^{3}}{\left(3 \times 10^{10}\right)^{2}} \\
& {\left[\frac{1}{\exp \left(6.63 \times 10^{-34} \times 2.75 \times 10^{12} / 1.38 \times 10^{-23} \times 617\right)-1}\right] } \\
& \times 3.47 \times 10^{7} \\
= & 5.60 \times 10^{-8} \mathrm{~J} \mathrm{~cm}^{2} \mathrm{sec}^{-1}
\end{aligned}
$$

We can also calculate the intensity from spontaneous decay. From the $A$ coefficient given in (5) and the information in Table (2) we have for the 13p-11d transition,

$$
\begin{aligned}
& A=0.6669 \times(91.6)^{2} \times 2.29 \\
& =1.28 \times 10^{4} \mathrm{sec}^{-1}
\end{aligned}
$$

The density of excited states, in the limit of saturation is,

$$
n_{0}=n_{t} / 2=1.56 \times 10^{16} \mathrm{~cm}^{-3}
$$

After 10 nanoseconds we see that the number of atoms $/ \mathrm{cm}^{3}$ left in the excited state is,

$$
\begin{aligned}
n(t) & =n_{0} \text { eAt } \\
& =\left(1.56 \times 10^{16}\right) \exp \left(-1.28 \times 10^{4} \times 10^{-8}\right) \mathrm{cm}^{-3} \\
& =1.5598 \times 10^{16} \mathrm{~cm}^{-3}
\end{aligned}
$$

or the number of decays is

$$
n_{d}=n_{o}-n(t)=2.00 \times 10^{12} \mathrm{~cm}^{-3}
$$


Each of these photons has the energy,

$$
E=h \nu=1.80 \times 10^{-21} \mathrm{~J}
$$

So we have a total energy flux out of the system of

$$
\begin{aligned}
F & =n_{d} E c \\
& =1.08 \times 10^{2} \mathrm{~J} \mathrm{~cm}^{-2}-\mathrm{sec}
\end{aligned}
$$

We see that this is much larger than (43a) and will dominate.

This energy is going into $4 \pi$ steradians. We would like to look at the energy flux going into the system to stimulate other atoms to emit. To calculate the percentage of $F$ stimulating further emissions, we first note that from $(40),(45)$, and

$$
\ell=\frac{1}{\sigma^{\mathrm{s} T_{n}}}
$$

for the characteristic length before the stimulated emission occurs, we have,

$$
\ell=8.66 \times 10^{-9} \mathrm{~cm}
$$

This is exceedingly short, since the internuclear distance at this pressure is $\sim 5 \times 10^{-6} \mathrm{~cm}$. We may consider all $4 \pi$ steradians of the flux in a calculation for the total stimulated rate. So, from (41), (39), and (48),

$$
\begin{aligned}
W & =B I \omega \\
& =B F / \Delta \nu \\
& =8.19 \times 10^{15} / \mathrm{sec} .
\end{aligned}
$$


where we have used the linewidth $\Delta \nu$ in (39) because it is the characteristic linewidth for the spontaneous emission involved in $I_{\omega} \cdot$

This is the initial stimulated emission rate and will grow as the total energy emitted from this transition grows. We see that this rate is very fast and we will in practice be limited by the pump rate and duration of the laser used to pump the 13p upper excited state.

We should also note here that we are de-exciting the upper state much faster by stimulated emission than by any other method (i.e. spontaneous emission or collisions.) Therefore, any rate equations would be dominated completely by the stimulated emission rate.

To get a better feeling for the process, we should compare the time $t$ when the stimulated rate exceeds the collisional rate. We, therefore, want to solve for $t$ in the equation,

$$
\begin{aligned}
\Delta \nu_{c}= & W(t) \\
& =B I_{\omega}(t) \\
& =B F(t) / \Delta \nu \\
& =B n d(t) E C / \Delta \nu \\
& =B E C / \Delta \nu \quad\left(n_{0}-n_{0} e^{-A t}\right)
\end{aligned}
$$

Using the values of these variables given in eqs. (38), (39), (41), (44), and (45), we have,

$$
\begin{aligned}
& 2.69 \times 10^{7} / \mathrm{sec}=4.09 \times 10^{3}\left(1.56 \times 10^{16}-1.56 \times 10^{16}\right. \\
& \left.e^{-1.28 \times 10^{4 t}}\right)
\end{aligned}
$$


or

$$
4.21 \times 10^{-13}=1-\mathrm{e}^{-1.28 \times 10^{4} \mathrm{t}}
$$

which yields

$$
t<1 \times 10^{-14} \sec
$$

To more accurately describe this system, one must write the complete set of rate equations and solve them for the photon population as a function of time. This method is outlined extensively in Refs. $(26,27)$ and will not be explored here. This method would give an accurate picture of the temporal behavior af the system, but because of the extremely large stimulated emission probability for our system, we would learn very little from the exercise.

One liseful result from this type of calculation is the population density required in the upper state to get above threshold. As shown in Ref. (26), we can write this threshold population density as,

$$
n_{\text {th }}=\frac{8 \pi}{3} \quad \frac{\Delta v}{\nu} \quad \frac{\tau \mathrm{rad}}{{ }^{7} \mathrm{c}}\left(\frac{1}{\lambda}\right)^{3}
$$

Where $\tau$ rad is the spontaneous lifetime of the state and $\tau_{c}$ is the cavity lifetime. In our case the cavity lifetime is the time light takes to travel one length of our excited state column. This is,

$$
\begin{aligned}
\tau_{c} & =\frac{15 \mathrm{~cm}}{3 \times 10^{10} \mathrm{~cm} / \mathrm{sec}} \\
& =5 \times 10^{-10} \mathrm{sec}
\end{aligned}
$$


Solving (54) for the 13p-11d transition we obtain,

$$
n_{t h}=1.17 \times 10^{7} / \mathrm{cm}^{3}
$$

Comparing this with (45) we see that we are quite far above threshold under the conditions stated. This equation is derived for a steady state inversion and we must, therefore, approximate our laser as a square wave with a steady state level for approximately 8 nsec. This is actually a reasonable approximation and yields, for a pump rate to be above threshold,

$$
\begin{aligned}
R_{\text {th }} & =\frac{n t h}{t}=\frac{1.27}{8 \times 10^{-9}} \text { Atoms } / \mathrm{cm}^{3}-\mathrm{sec} \\
& =1.59 \times 10^{15} \text { Atoms } / \mathrm{cm}^{3}-\mathrm{sec}
\end{aligned}
$$

If we use the energy per photon to excite the upper state.

$$
\begin{aligned}
h i & =6.63 \times 10^{-34} \times 1.02 \times 10^{15} \\
& =6.76 \times 10^{-19} \text { Joules/photon }
\end{aligned}
$$

and typical operating parameters of

$$
\begin{aligned}
& d=\text { beam diameter }=.2 \mathrm{~cm} \\
& l=\text { column length }=15 \mathrm{~cm} \\
& \text { Ep }=\text { Pump energy }=6 \times 10^{-6} \text { Joules } / \text { pulse, }
\end{aligned}
$$

our available pump rate is,

$$
R_{p}=\frac{E p}{h \nu} \frac{1}{\pi\left(\frac{d}{2}{ }^{2} 1\right.} \frac{l}{t_{p}}
$$

where $t_{p}$ is the pulse duration of $8 \mathrm{nsec}$. This yields 


$$
R_{p}=2.35 \times 10^{21} / \mathrm{cm}^{3}-\mathrm{sec}
$$

which is much larger than the threshold pump rate in (5?). We should, therefore, expect the system to operate in the stimulated emission mode when pumped as stated above.

So far we have discussed only the pump transition 4s-13p and the desired :IR emission transition 13p-11d. We have assumed a population inversion in the upper state (quite reasonable since our thermal energy is $\sim 432 \mathrm{~cm}^{-1}$ compared to the $34000 \mathrm{~cm}^{-1}$ lower lasing level) but have not examined the depopulation processes for the lower level. These would include spontaneous emission, stimulated emission, and level changing collisions. We see from Table (4) that the natural lifetime of the 11d state is $4.5 \mu \mathrm{sec}$, and therefore, will probably not be important in the total iifetime. In fact the arguments put forth for the 13p-11d transition can be invoked with virtually no change for the $11 \mathrm{t}-12 p$ and $11 \mathrm{~d}-10 \mathrm{f}$ transitions so we expect a very rapid depopulation of this level by stimulated emission with very little effect from collisions since the time between collisions is long (factor of 10) compared to the stimulated emission rate.

Therefore, the temporal behavior of the laser is expected to be completely controlled by the laser pump with the actual atomic processes happening at a rate that is so fast as to be unobservable.

To get an idea of the power out of the system in the FIR transition, we can make a very simple calculation based on the 
TABLE 4. Transitions from $11 d-n p$ and $11 d-n f$ with the A coefficients and lifetimes as well as the total A coe 'sicient.

\begin{tabular}{|c|c|c|c|}
\hline Transition & $f_{i k}$ & $\left(E_{k}-E_{j}\right) c_{1}^{-1}$ & $A_{i k} \sec ^{-1}$ \\
\hline $11 d-12 p$ & 1.95 & 83.96 & 9167.27 \\
\hline $11 d-11 p$ & .0352 & 319.46 & 2393.00 \\
\hline $11 d-10 p$ & .00437 & 645.36 & $1.215 \times 10^{3}$ \\
\hline $11 d-9 p$ & .000714 & 1114.82 & $5.918 \times 10^{2}$ \\
\hline $11 d-8 p$ & .0000672 & 1826.78 & $1.500 \times 10^{2}$ \\
\hline $11 d-7 p$ & .00000744 & 2982.44 & $4.413 \times 10^{1}$ \\
\hline $11 d-6 p$ & .000103 & 5049.20 & $1.744 \times 10^{3}$ \\
\hline $11 d-5 p$ & .000247 & 9336.70 & $1.434 \times 10^{4}$ \\
\hline $11 d-4 p$ & .000397 & 21014.01 & $1.160 \times 10^{5}$ \\
\hline $11 d-10 f$ & $1.00 *$ & 146.7 & $1.435 \times 10^{4}$ \\
\hline $11 d-9 f$ & .113 & 404.9 & $1.235 \times 10^{4}$ \\
\hline $111-8 f$ & .0319 & 765.86 & $1.248 \times 1 n^{-1}$ \\
\hline $11 d-7 f$ & .0118 & 1292.38 & $1.314 \times 10^{f}$ \\
\hline $11 d-6 f$ & .0047 & 2103.9 & $1.387 \times 10^{4}$ \\
\hline $11 d-5 f$ & .00175 & 3451.3 & $1.390 \times 10^{4}$ \\
\hline $11 d-4 f$ & .000476 & 5929.2 & $1.116 \times 10^{4}$ \\
\hline
\end{tabular}

$$
\begin{aligned}
\left.A_{i} \text { (ТОT }\right) & =2.369 \times 10^{5} \mathrm{sec}^{-1} \\
\tau_{j} & =4.22 \times 10^{-6} \mathrm{sec}
\end{aligned}
$$

*extrapoiation from reference (2) 
Manley-Rowe limit. This is, for every photon absorbed one photon is emitted in the FIR.

Assuming now $1 \mu 3$ of incident jump power far pulse, if we are at the Manley-Rowe limit, we would expect a signal corresponding to,

$$
\begin{aligned}
E_{F I R} & =E_{p} \frac{\nu F I R}{\nu p} \\
& =1 \times 10^{-6} \frac{2.75 \times 10^{12}}{1.02 \times 10^{15}} \\
& =2.70 \times 10^{-9} \mathrm{~J} \mathrm{pulse}^{-1}
\end{aligned}
$$

which corresponds, in the limit of a square wave, to

$$
\begin{aligned}
P_{F I R} & =\frac{E_{F I R}}{t} \\
& =\frac{2.70 \times 10^{-9}}{8 \times 10^{-9}} \mathrm{J \textrm {sec } ^ { - 1 }} \\
& =337 \mathrm{~m} \text { Watts }
\end{aligned}
$$

for the peak power in the system.

The system is not controlled by a cavity geometry so we would expect less than the power indicated in (62) to exit the device through the output end. The fraction exiting this way will be determined by both the aspect rivio of the amplifying medium and by the rate of stimulated emission, as this will determine how much effect neighboring atums have on each other. From a purely geometric consideration, we can argue that only that portion of 
the FIR traveling down the column will be amplified, and therefore, we should expect,

$$
a=\frac{\ell}{d}=75
$$

to be the ratio of the power out along the column to that which goes out in other directions. This means that $98.7 \%$ of the radiation will exit colinear to the column with a divergence associated with this aspect ratio of $\frac{1}{a}=0.013$ radians. One can argue here that because of the extremely fast stimulated emission rate, we would only expect the FIR to exit in one direction, the direction the pump beam is traveling, because the stimulated emission will happen almost instantaneously after the medium has been excited.

\section{Stark Effect}

The second major theoretical area to be explored is the response of a highly excited atomic system to an applied electric field. Recently a detailed study of the Stark structure in the alkalis has been done with emphasis on the $n=15$ levels (28). This work outlines the theoretical methods used to do detailed calculations on the Rydberg states of the alkalis and gives results for the $n=15$ manifold of potassium. Here we will review the results and apply them to the system levels of interest.

We begin by writing Schrodinger's equation for the atom in a static electric field, neglecting spin, 


$$
\left[-\frac{1}{2} \Lambda^{2}+V(r)+F_{z}\right] \psi(\bar{r})=W \psi(\bar{r})
$$

where $W$ is the energy and $F$ is the magnitude of the electric field which is taken to be along the z-axis. $V(r)$ is not known and the Hamiltonian is not separable so no analytical solution is possible. Because all levels are changing drastically with time a perturbation method is not suited to the problem. Constructing the wave functions by the use of Matrix diagonalization is, therefore, the most appropriate path to follow. Two approximations which prove very helpful and provide good results are; outside of the electron core, the potential $V(r)$ is essentially Coulombic and the off-diagonal matrix elements decrease very quickly as the energy difference between the states these represent increases. Therefore, only those elements that are near the level of interest need be included in the evaluation of the matrix.

Since the unperturbed energies for the alkalis are diagonal in the spherical representation and the Stark effect is not diagonal in the parabolic basis, it is more straightforward to work in the spherical representation.

The matrix elements of the Stark interaction may be written

$$
\begin{aligned}
& <\omega, \ell, \mathrm{m} / \mathrm{Fz} / \mathrm{W}^{\prime}, \ell^{\prime}, \mathrm{m}^{\prime}>= \\
& \delta_{\mathrm{m}, \mathrm{m}^{\prime}} \quad \delta_{\ell, \ell^{\prime} \pm 1} \mathrm{~F}<\ell, \mathrm{m} / \operatorname{Cos} \theta / \ell^{\prime} \mathrm{m}^{\prime}><\omega, \ell / \mathrm{r} / \omega^{\prime}, \ell^{\prime}>
\end{aligned}
$$

We have the usual result for the angular portion using spherical harmonics, 


$$
\begin{aligned}
& <\ell, \mathrm{m} / \cos \theta / \ell-1, \mathrm{~m}>=\left[\frac{\ell^{2}-\mathrm{m}^{2}}{(2 \ell+1)(2 l-1)}\right]^{1 / 2} \\
& <\ell, \mathrm{m} / \cos \theta / \ell+1, \mathrm{~m}>=\left[\frac{(\ell+1)^{2}-\mathrm{m}^{2}}{(2 \ell+3)(2 \ell+1)}\right]^{1 / 2}
\end{aligned}
$$

The radial part of the wavefunction is all that remains to be evaluated and this has been done in Ref. (28) using numerical integration methods.

As mentioned in (28) the fine structure does not become important until the heavier alkalis. In the case of potassium we do not need to consider it, as it is much less than the $.3 \mathrm{~cm}^{-1}$ resolution of our experimental apparatus.

The theoretical methods in (28) then provide us with a method for caiculating the energy level shifts to an accuracy of $<.05$ $\mathrm{cm}^{-1}$. Recently this method has been extended to the $\mathrm{n}^{\star}=11$ manifold of potassium (29) and the results of these calculations are shown in Table (5). We have shown here only those states that are of interest in our application although calculations for all values of 1 have been done. In Table (6) we see the shifts expected at 2000 volts/cm for the three t:ansitions observed. We can write

$$
E=E_{0}+\alpha F^{2}
$$

where $\alpha$ is the polarizability of the levet in $\mathrm{cm}^{-1} /(\mathrm{KV} / \mathrm{cm})^{2}, F$ is the field strength in $\mathrm{KV} / \mathrm{Cm}$ and $E_{0}$ is the unperturbed energy of the lever. Using the convention for energy levels in (29) we have, 
TABLE 5. Results from the calculations by Zimmermar, on the potassium Stark shift.

$$
M=0
$$

State Field KV/cm

$14 \mathrm{~s}$

$13 p$

135

$11 d$

$12 \mathrm{p}$

$13 p$

$11 \mathrm{p}$

$12 p$

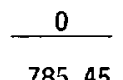

785.45

860.93

937.35

953.14

1036.39
1.5

785.82

861.22

937.55

953.67

1036.55

2037.05

$$
M=1
$$

860.93

861.18

861.93

953.14

953.62

955.09

1036.39

1036.53

1036.94 
TABLE 6. Potassium shifts for $2000 \mathrm{~V} / \mathrm{cm}$.

Transition

$14 s-13 p$

$13 p-11 d$

$11 d-12 p$

\section{Prodicted}

$-.11 \mathrm{~cm}^{-1}$

$+.42 \mathrm{~cm}^{-1}$

$-.66 \mathrm{~cm}^{-1}$
Observed

$-.59 \pm .15 \mathrm{~cm}^{-1}$
$.43 \pm .15 \mathrm{~cm}^{-1}$
$-.24 \pm .15 \mathrm{~cm}^{-1}$ 


$$
\begin{aligned}
& \alpha(13 p)=0.131 \mathrm{~cm}^{-1} /(\mathrm{KV} / \mathrm{cm})^{2} \\
& \left.\alpha(11 \mathrm{~d})=0.237 \mathrm{~cm}^{-1} / \mathrm{KV} / \mathrm{cm}\right)^{2} \\
& \alpha(1 \mathrm{ss})=0.159 \mathrm{~cm}^{-1} /(\mathrm{KV} / \mathrm{cm})^{2} \\
& \left.\alpha(12 \mathrm{p})=0.073 \mathrm{~cm}^{-1} / \mathrm{KV} / \mathrm{cm}\right)^{2}
\end{aligned}
$$

but

$$
\begin{aligned}
\Delta E & =E-E_{0}=\alpha F^{2} \\
\Delta E_{S} & =\Delta E_{k}-\Delta E_{j}=\left(\alpha_{k}-\alpha_{i}\right) r^{2}
\end{aligned}
$$

so for the $13 p \rightarrow 11 d$ transition

$$
\Delta E_{s}(13 p-11 d)=0.106 \mathrm{~F}^{2} \mathrm{~cm}^{-1}
$$

similarly

$$
\Delta E_{s}(14 s-13 p)=-0.028 F^{2} \mathrm{~cm}^{-1}
$$

and

$$
\Delta E_{s}(11 d-12 p)=-0.164 F^{2} \mathrm{~cm}^{-1}
$$

Equations (69), (70), and (71) then give us the direction and magnitude of the shifts expected in the energies of these transitions. These shifts will be the observables in the experiment as well as the absolute shift of the upper pumped level as determined by the wavelength change of the pump laser. 


\section{EXPERIMENTAL}

In the process of developing this FIR source, many different configurations for pumping the upper level of the atom were considered. Some of these will be described here with special emphasis on those that show some potential for further development. He will also describe a device known as a heat pipe oven, that has made possible the confinement of hot potassium vapor while still keeping optical access to the vapor. Finaliy the series of experiments demonstrating the generation of the FIR and then the tuning of the FIR will be described in detail.

As mentioned in the introduction, this work was prompted by results of experiments done at the IBM labs by Wynne \& Sorokin (30) and by a proposal-type paper written by Lau et a1. (31). In these papers C. W. operation of a FIR laser was postulated and puised operation demonstrated.

Early in the experimental phase of the work, it was decided that the stimulated emission results in (30) should be reproduced. This was done for two reasons. We wanted to get a measure if familiarity with the hardware involved in such experiments and a baseline was needed from which we could compare the results of later modified systems.

Heat Pipe

The first problem with the experimental work was the confinement of the alkali metal vapor at temperatures of up to $450^{\circ} \mathrm{C}$. To address this problem we will examine the methods used to contain hot metals previous to 1970 . 
The most common system was one of a hot furnace with some combination of heated windows, baffles, or inert gas jets to keep the windows clear. These methods, however, all suffer from their own set of probleris and one generally would end up with a poorly characterized vapor region that was not stable spacially or temporally. Sophisticated solutions to these problems have been used but with limited success.

Another cormon technique for the generation of pure metal vapor is the use of an atomic beam. This technique gives a collision free vapor on which to do spectroscopy, but is limited by the techniques needed to characterize the vapor and by the very low density of the vapor (typically $<10^{12}$ atoms/cc). In a system where we expect to need a large number of atoms for the active medium, a beam would seem unsuited to the task.

Fortunately an alternative to these methods was developed by Vidal and Cooper (32) in the form of the heat pipe oven. This $d f . v^{\prime} c e$ is based on the heat pipe developed by Grover et al. (33) for the transfer of heat. The system uses the heat of vaporizion of the metal as the heat transport mechanism and relies on the surface tension of the liquid metal to return the liquid to the heat source for recycling. A schematic of a simple heat pipe oven is shown in Figure (4). We see that it consists of a heater, cooling coils, stainless steel screen which acts as a wick, and inert gas. The potassium metal is placed in the middle of the heat pipe and a vacuum is pumped on the pipe. This is done at an elevated temperature but below the melting point of the metal 


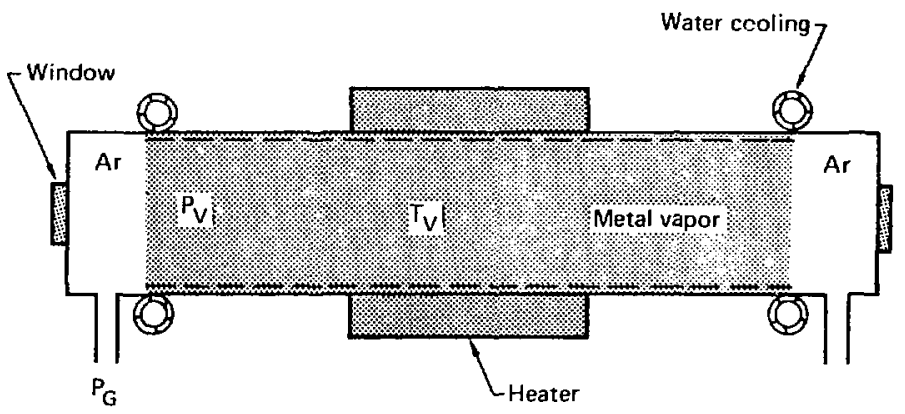

HEAT PIPE OVEN

Figure 4. A schematic diagram of a heat pipe oven. 
$\left(63.7^{\circ} \mathrm{C}\right)$ to remove any solvent used to clean the metal. We used cyclohexane for this purpose. When this step is complete, the pipe is filled with an inert gas, (in this case argon,) to the pressure at which the pipe will be operating. It is important to note here that the working pressure of the vapor will be determined solely by the pressure of the buffer gas. As more power is put into the heaters, the column length will increase. If the buffer gas reservoir is large the overall pressure will remain essentially unchanged. The hat metal atoms will collide itih cold buffer gas atoms and will condense on the wick and flow back to the heated region because of surface tension. We can, therefore, control the pressure of the vapor very accurately by controlling the pressure of the buffer gas which means contralling the temperature of the buffer gas. For a system with a large buffer gas reservoir, we need control only the room temperature well to have satisfactory control over the vapor pressure. As shown in (34) one need only control the temperature of the reservair to $2 \%$ to maintain the oven temperature to $0.1 \%$. Therefore, in our laser lab where the temperature is controlled to $22^{\circ} \mathrm{C} \pm 1^{\circ} \mathrm{C}$ or 4.5\%, we expect the oven to be controlled to better than .3\%. As mentioned above, by adding heat to the system we do not increase the pressure of the system but rather increase the length of the vapor column. He can, therefore, change the density and size of the vapor accessed independently. This can be of great advantage when optimizing the experimental configuration. The boundary layers between the vapor and the buffer gas are extremely narrow 
and constitute a small fraction of the total length of the vapor column Figure (5).

Our heat pipes have been operated with pressures of 0.1 to 10 Torr $\left(270^{\circ} \mathrm{C}\right.$ to $\left.450^{\circ} \mathrm{C}\right)$ for up to 500 hours without window problems. The changing of windows was initially done in an argon filled glove box but. later it was found sufficient, by observation of the potassium oxidation, to continuously flush the heat pipe with argon gas as the window was changed. Wiridow materials included sapphire, quartz, polyethylene, and pyrex. All made suitable windows for spectral areas of interest.

The heat pipe illustrated in Figure (4) consisted of a 3/4" stainless steel ( 316 stainless) pipe $50 \mathrm{~cm}$ in length. The windows were attached by way of conflat varian flanges with copper gaskets and the wick material was 100 mesh stainless steel screen. The cooling coils were 3 turns of $1 / 8^{\prime \prime}$ copper tubing and the heater was a 200 watt heat tape controlled by a Variac variable transformer. All pressure measurements were done with an MKj capacitive monometer with a 1000 Torr head. Temperature measurements were done with an iron-constantan (copper-nickel) thermocoup le.

This heat pipe was used for most of the experiments described here but did not have the capability for the most important experiment, that of tuning the FIR output of the device. For this job a heat pipe with electric field plates was necessary and construction of two models were undertaken. The two models were drastically different in appearance, but quite similar in 


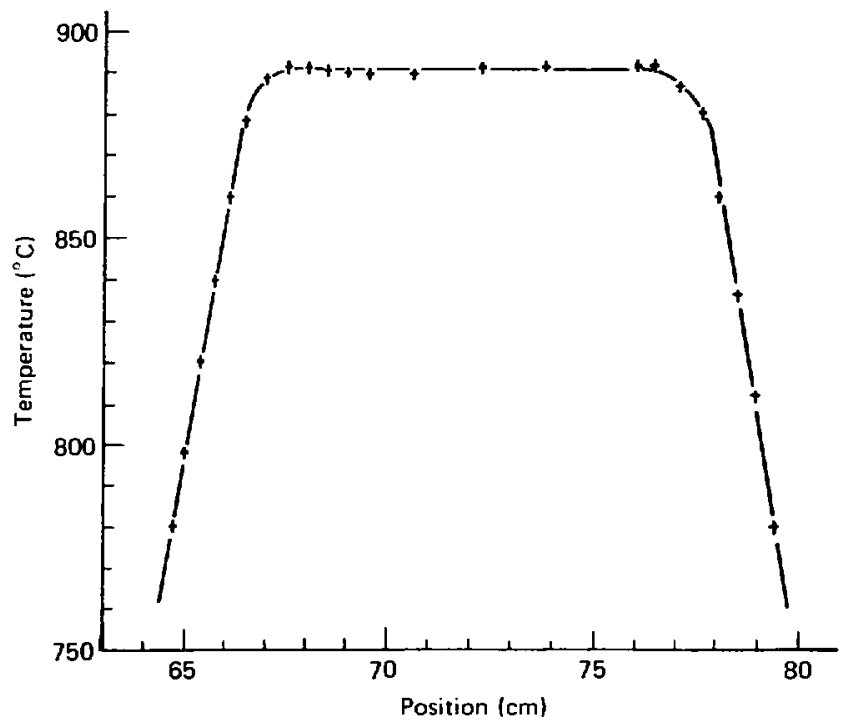

Figure 5. The temperature profile of a heat pipe containing lithium. (32) 
potential usefulness. The first model illustrated in Figure (6) was designed to have heated Stark plates which would be held approximately $5^{\circ} \mathrm{C}$ above the potassium reservoir temperature to keep the vajor from condensing on the plates. The plates would be totally inside the vapor region, being supported from the top by stainless-steel tubes which allow the heater and thermocouple wires to exit. The thermocouples were chromel-alumel (nickelchromium, nickel-aluminum) and the heaters were controlled by a variac with isolation transformers to prevent an electrical feedback of th. applied d.c. voltage into the a.c. line. A photograph of the completed heat pipe is shown in rigure (7). There are two small ports in the top of the plate support cover. One of these was used to view fluorescence in the ripe and the other was usej to place a thin (1/8" diameter) stainless-steel electroci- near the top of the plates to be used to detect ions formed between the electrodes. In the case where the Stark plates were not in use, one could use this electroje to probe for any ionized atoms formed by ionizing collisions of the highly excited atoms. A small voltage applied to this electrode would '"en induce a current that could be measured with a picoammeter. When ihe Stark plates were in use th: current flowing in that system would be an indication of any ionizing processes taking place. This heat pipe was designed with a $0.5 \mathrm{~cm}$ spacing between the plates and did not have a stainless-steel screen for iicking. The potassium liquid was to be returned by gravity feed. The windows of the heat pipe were attached to flexible stainless steel bellows 


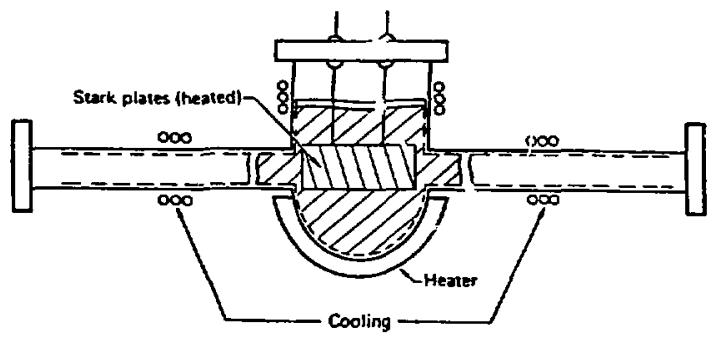

Figure 6. A schematic diagram of the "can" type heat pipe oven with Stark plates. 


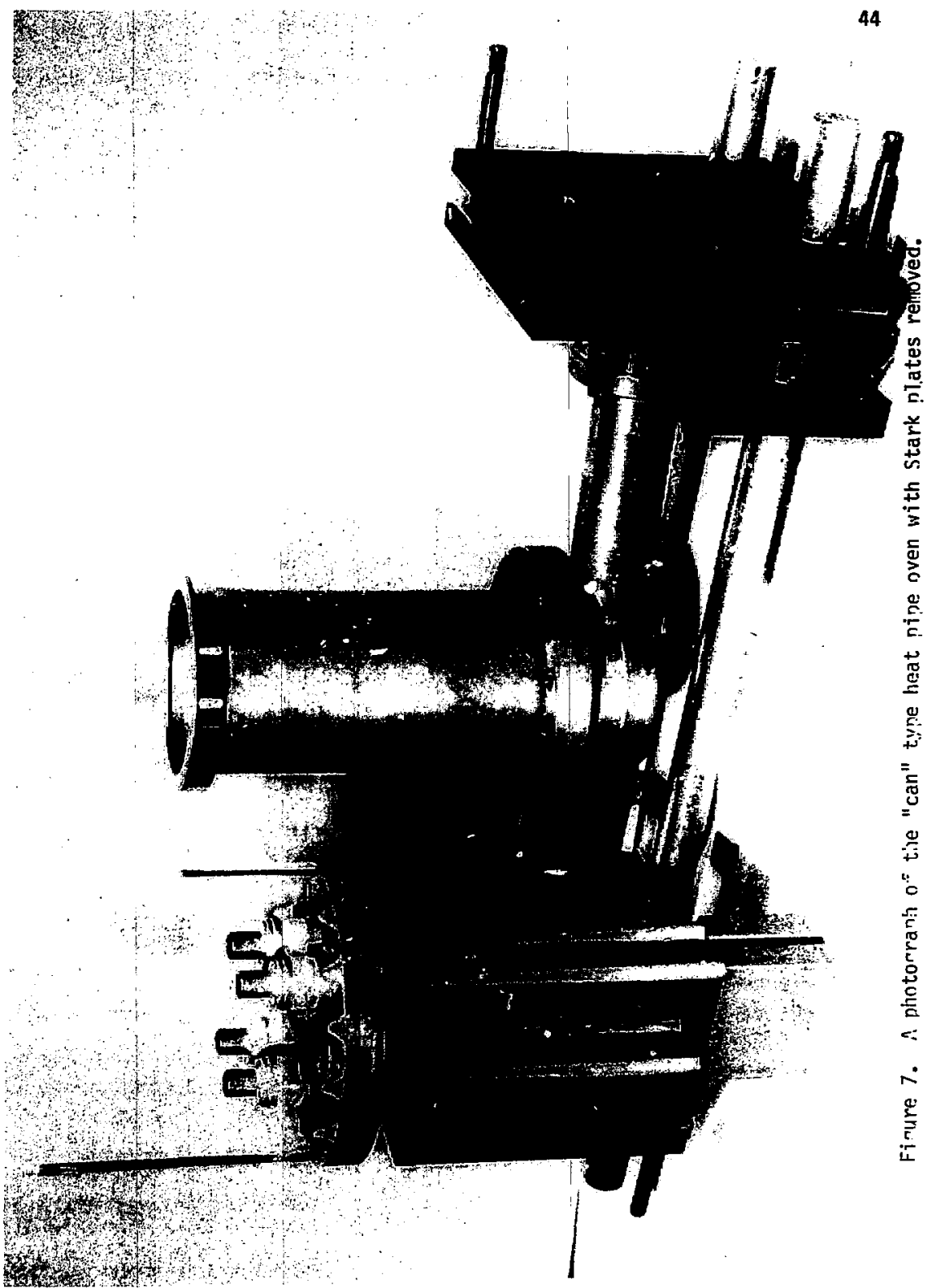


to allow the windows and hole coupled intra-pipe mirrors to be adjusted. The entire pipe assembly was cradled in a base consisting of aluminum brackets supported by invar steel rods 1 " in diameter. The heater for the main pipe consisted of a mantle heater under the pipe and a 200 watt heat tape wrapped around the upper portion of the pipe and around the inner portion of the arms. The cooling consisted of 1/4" i.d. copper tubing wrapped twice around each arm about three inches from the main tube and around the main tube about four inches from the top. The main tube is sir inch diameter 316 stainless steel and the arms are one and one-half inch 316 stainless steel inserted into the main tube at a $10^{\circ}$ slope to allow the liquid potassium to flow back to the main reservoir area. A plexiglass hoct was built to cover the exposed electrical connections to the field plates and all electrical connections were made through a single cable to the hood. A chassis was constructed to house the four thermocouple readouts (one for each cartridge heater in the plate supporting arms), the variac to contral the cartridge heater, and the isolation transformer.

A potential problem with this design is that not all of the alkali metal vapor in the column defined by the pump iaser is between the plates. At each end of the plates there is vapor that would be in either a very inhomogeneous field or zero fieid when voltage is applied to the plates. Fortunately this is not a significant problem because of the Stark response of the potassium vapor. The upper state is an $1=1$ state and as seen in Chapter 
II is shifted strongly in an electric field of moderate strength. Therefore: to continue to pump this upper level, we have to tune the pump laser back into resonance with the now-shifted upper state. This means that only that portion of the metal vapor that has been shifted will absorb the pump laser light and we have effectively circumvented the problem.

The second model of heat pipe designed for use with electric fields is quite different in design from the one just described. It consists of two large plates separated by an insulating element as illustrated in Figure (8). This device is very similar in design to one described in Ref. (35). It consists of two 316 stainless-stee 1 plates $17^{\prime \prime} \times 17^{\prime \prime} \times 5 / 8^{\prime \prime}$. These are separated by a stainless-steel ring and are both insulated by 1/4" 0.D. 0-ring gaskets. There is also an epoxy composite insulating standoff between the plate and the ring on the non-load bearing surface Figure (9). One can think of this device as simply a normal heat pipe oven with $360^{\circ}$ symmetry about an axis normal to the plane of the plates. Therefore, instead of having a working vapor in the shape of a column we have the vapor in a disk shape. Windows were placed conveniently around the pipe so a variety of measurements could be made with no need for changing windows. A sapphire input window was used to allow access to the pipe by both visible and U.V. radiation. Output windows used were $Z$-cut quartz and polyethylene to allow visible, U.V., and FIR radiation to be monitored. Both output windows were attached to the same side of the pipe and were separated by approximately $5 \mathrm{~cm}$. The output 


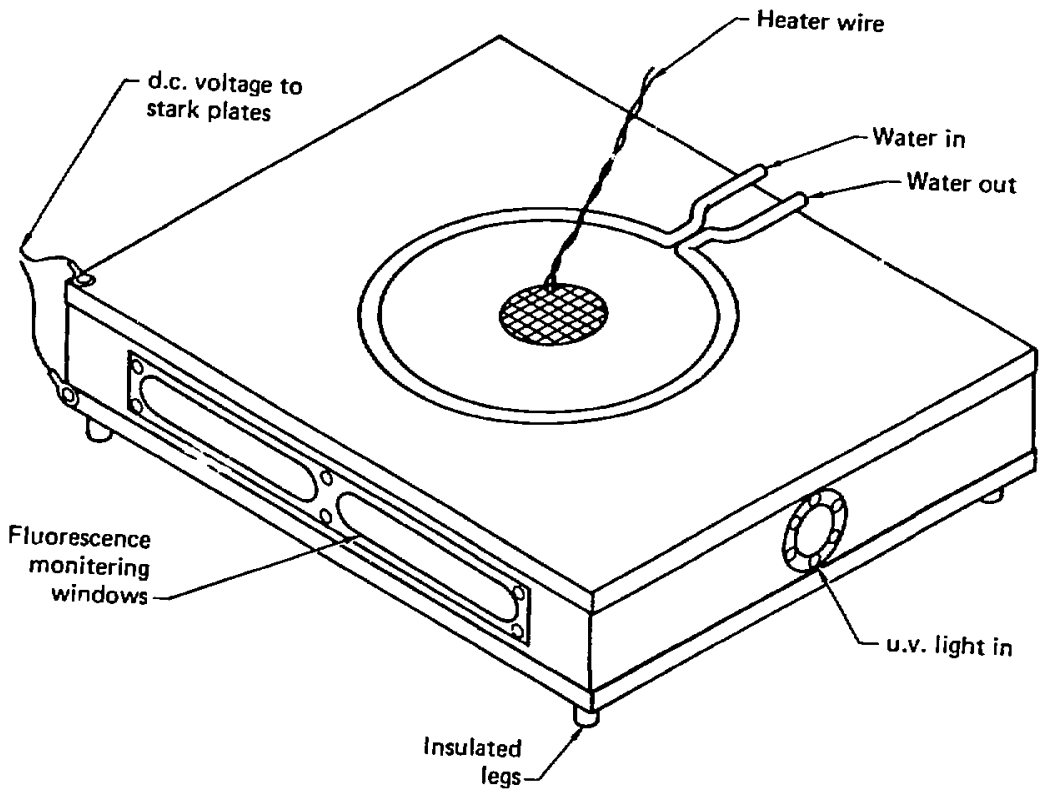

Figure 8. Sketch of the "pancake" heat pipe oven. 


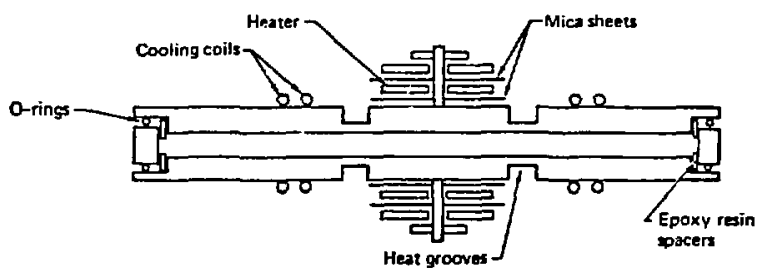

Figure 9. Cross section view of the "pancake" heat pipe oven. 
could be monitored through either window by simply rotating the pipe. The very useful added $f($ ature of this heat pipe was the window which ran along the length of one of the walls adjacent to input and output windows. This window was approximately $1.5 \mathrm{~cm}$ high and $33 \mathrm{~cm}$ long and gave a clear side view of the excited alkali metal vapor when the pump laser operated. This aided alignment enormously and helped identify resonances quickly. Two heaters were used, one on each plate. They were 750 watt Kame?rok model 63102, 5 inch diameter ring type heaters and were insulated electrically (and unfortunately partially thermally) from the plates with a .004 inch thick mica sheet. A bolt was werded to the plate and a $1 / 2$ inch thick aluminum plate was used to hold the heater to the stainless-steel heat pipe plate. This was also insulated from the heater by a mica sheet. All surfaces were coated with nonconductive heat sink compound to aid thermal transfer. Both heaters were controlled independently by variacs and the temperature of the stainless-steel plates were monitored by iron-constantan thermocouples. Cooling coils were made of $1 / 4$ i.d. copper tubing and were formed into $30 \mathrm{~cm}$ diameter circles of two windings and were soldered onto the stainless-steel plates. Heat sink compound was also used on them to insure good thermal contact. These were connected to the Low Conductivity water (LCW) supply by plastic hose. The LCW supply is a closed laboratory wide source of cooled deionized water which has, as its name implies, very low electrical conductivity. The two plates were held together with approximately $20 \mathrm{C}$-clamps which were insulated 
from the plates by $1 / 8^{\prime \prime}$ thick epoxy resin board. The separating ring was insulated from both plates as stated above and was insulated from the vacuum system by the insertion of a glass transition in the vacuum line. The wicking of the upper plate consisted of a single layer of 100 mesh stainless-steel spot welded directly to the plate. The lower wick consisted of 100 mesh stainless-steel spot welded to a $1 / 8$ inch thick stainless steel plate which was then bolted to the bottom plate. This was done to allow access to the potassium metal reservoir which consisted of a $3 / 8$ inch deep area 2 inches square machined into the bottom piate. When loading the pipe, the potassium was set in the machined out area and the thinner plate bolted on top of it. This allowed us to keep all high melting point contaminants out of the working area. Several holes were drilled in the $1 / 8$ inch plate above the reservoir area to allow the potassium vapor to enter the working area. In addition, to prevent thermal doming in the $1 / 8$ inch plate, a circle of approximately $30 \mathrm{~cm}$ diameter was cut and the cut out piece was replaced and held into place by the screen. This left a $1 / 16$ inch gap around the entire section which allowed the disk to expand without buckling when heated. The plate spacing was approximately $1.3 \mathrm{~cm}$ before heating but the doming effect of the iarge plates reduced this to less than $1 \mathrm{~cm}$. The exact distance was not measured when hot. The uniformity of the field was only good to $15 \%$ due to this effect. To prevent thermal transfer through the plate outwards, a thermal groove was cut in a square pattern about $20 \mathrm{~cm}$ on each side centered about 
the center of the plate and approximately $3 / 8$ inch deep. This groove and the cooling coils kept the edges of the heat pipe below $60^{\circ} \mathrm{C}$. At no time was fogging of the windows observed. The electric field was applied directly to the plates and was used in both a c.W. and pulsed mode. The lower plate was attaches to ground and supported on the laser table by four lab jacks. The upper plate held the high voltage and the middle ring was floated to provide more uniform fields. Care was taken to pump a good vacuum on the system (approximately $10^{-6}$ Torr) before loading and whenever the heat pipe was cold. This eliminated any oxide build-up on the potassium. Loading was accomplished by placing thin (1/4 inch) potassium metal slices in the reservoir which was filled with cyclohexane to prevent oxidation of the potassium. A vacuum was then pumped on the system to evaporate the cyclohexane and heat was applied to increase the rate of evaporation. When the system held the vacuum satisfactorily, argon gas was put in at a suitable pressure $(\sim 1$ Torr $)$ and the device was heated to a temperature appropriate to the pressure $\left(332^{\circ} \mathrm{C}\right.$ at 1 Torr $)$. Configurations

In the course of the theoretical work, it became obvious that there were several ways to experimentally achieve the desired population inversion in the potassium vapor. Many different pumping schemes were explored on paper and some were tried experimentally in the laboratory. Here we will simply explain the experimental configurations tried and will leave the discussion of 
the experiments done with these configurations to the last section of this chapter.

The first configuration tried is the same as in Ref. (30). We used a Molectron Model U.V. 1000 nitrogen laser to pump a Molectron D. L. 400 dye laser. The only element in the cavity to reduce the dye laser. linewidth was a telescope to expanr the beam on the grating and thereby improve the number of grating lines illuminated which narrows the dye las rr output. This gives us a dye output bandwidth of $.5 \mathrm{~cm}^{-1}(15 \mathrm{GHz})$. Pulses from the dye laser are typically $8 \mathrm{nsec}$ in duration with an output energy of $500 \mu$ Joules per pulse at a repetition rate of $10 \mathrm{~Hz}$ using Rodamine $6 G(R 6 G)$ as the active lasing dye. The power spectrum of this dye is shown in Figure (10). We see that the peak output is at 580 $\mathrm{nm}$. Our measurements were all done at $585 \mathrm{~nm}$. To excite the 13p state of potassium, we need the second harmonic of the dye laser output. This wavelength is $\sim 292.5 \mathrm{~nm}$. To obtain the second harmonic one must focus the laser beam down onto a crystal with a high non-7inear index which effectively mixes the beam with itself and generates photons of twice the energy. For the wavelengths in which we are interested, the suitable crystal for the job is KDP (potassium dihydrogen phosphate) in an angle tuned configuration. Typical doubling efficiency was $3 \%$ which gave an output energy of $10-15 \mu$ joules per pulse in the U.V. with a pulse duration of approximately $4 \mathrm{nsec}$. The output beam was then re-collimated before entering the hert pipe. This configuration is illustrated in Figure (11). 


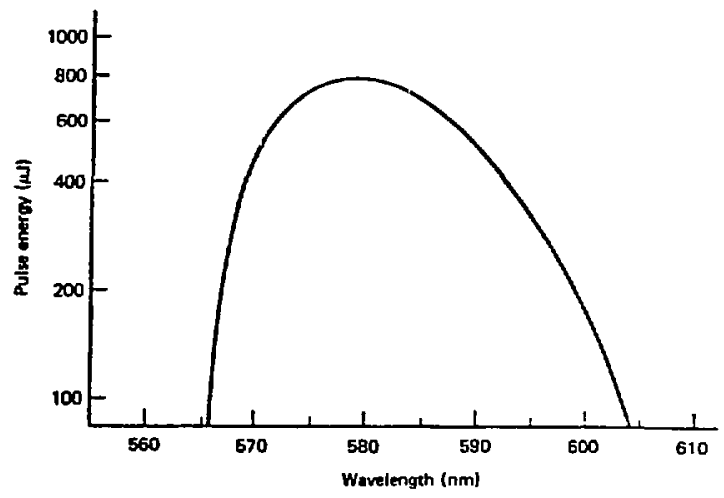

Figure 10. Power spectrum for R6G laser dye when used in a Molectron DL-200 laser pumped by a Molectron UV- $1000 \mathrm{~N}_{2}$ laser. 


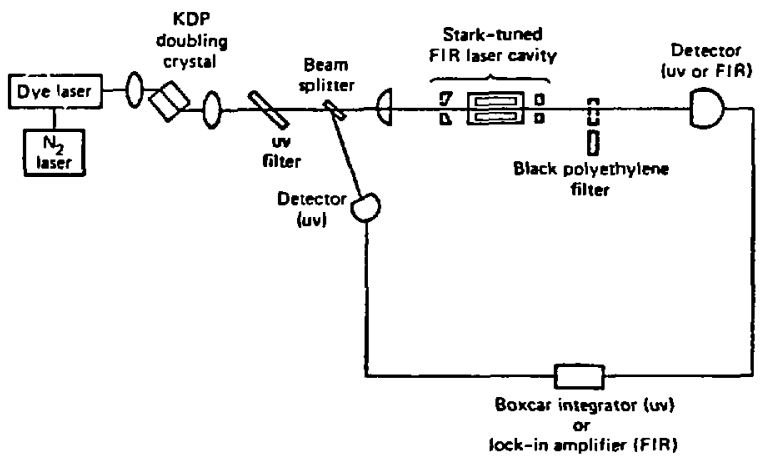

Figure 11. The experimental configuration used with the $\mathrm{N}_{2}$-Dye laser pump. 
The second configuration attempted was similar in that we attempted to pump the upper level with a single U.V. photon. In this configuration we used a Chromatix Moctl CMX-4 flasnlamp pumped dye laser. The overall energy from this system was substantially higher than in the Molectron system. Typical visible output was $1 \mathrm{~m}$ Joule at $585 \mathrm{~nm}$, but the pulse was now approximately $1 \mu \mathrm{sec}$ long. This system was much more efficient, however, in its production of U.V. light. The doubling crystal was placed inside the cavity and the output mirror of the laser was changed to a dichroic mirror which is very highly reflective in the visible, thereby keeping a very high finesse cavity for visible lasing, but also transmits greater than $80 \%$ of the ultraviolet light at the wavelength of interest. We, therefore, have the very high circulating power in the dye cavity to double with instead of the usual 20\% dye output. This system was routinely operated with a U.V. output of $200 \mu$ joules per pulse at a $10 \mathrm{~Hz}$ repetition rate with .5 $\mathrm{cm}^{-1}$ linewidth and $1 \mu$ second pulse duration. Because of the lang pulse length, the peak power of this pulse is only 200 watts compared to 2500 watts for the Molectron system. So the advantage is total energy emitted and the disadvantage is the peak power of the pulse. The Chromatix also had the minor advantage of a more compact unit. A schematic of this pump scheme is illustrated in Figure (12).

The next pump scheme uses a quite different approach to accessing the upper state of the atom. Instead of using a single photon of highly energetic light, which is quite costly to obtain 


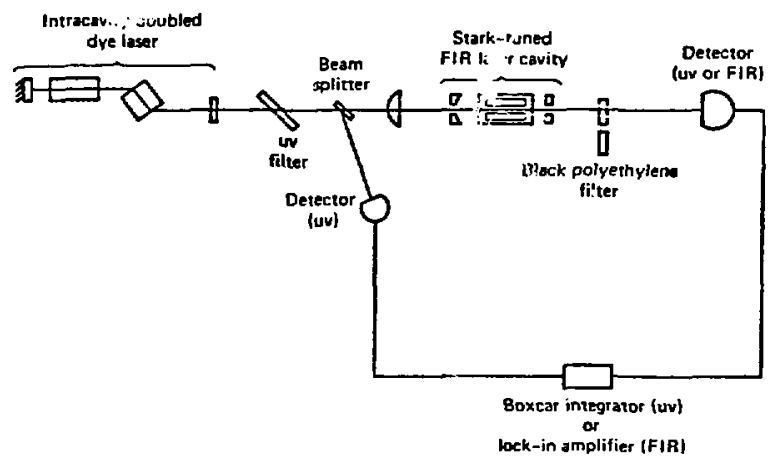

Figure 12. The experimental configuration used with the Chromatix CMX-4 dye laser purnp. 
(in terms of system efficiency), we could also use two photons of suitable wavelengths to sequentially pump a lower excited state and from there pump up to the sxcited state of interest. We should note here ihat the final state in the case of potassium would then be an $1=0$ or $1=2$ state, since the first excited state pumped wou?d be an $l=$ ? state. This would not be a problem, however, in the operation of the device. Since the final upper state in potassium would lie in the range of $34,000 \mathrm{~cm}^{-1}$ above the ground state, we would like to access a lower excited state very nearly half-way above the ground state. The first excited state in potassium lies at approximately $13,000 \mathrm{~cm}^{-1}$ above the ground state so we would, therefore, need a second photon of about $21,000 \mathrm{~cm}^{-1}$ or $475 \mathrm{~nm}$ wavelength to rearh the upper state. This second wavelength is quite easily accessible with a flashlamp pumped dye laser. He could also use a krypton pumped dye laser in the c.w. mode. Becaus: we only had one $\mathrm{Kr}^{+}$ laser available, and the pulsed system would not operate at 13,000 $\mathrm{cm}^{-1}(767 \mathrm{~nm})$ we could not operate in a completely c.w. or pulsed mode. An attempt was made to operate in a hybrid mode where we would pump the first excited state with a krypton ian dye laser using an oxazine dye followed by a pulsed flashlamp pumped dyc laser operating at $475 \mathrm{~nm}$ using the Exiton laser dye i.0. $4 ? 3$.

During this period of pump choice, many other systems were proposed. We will mention here one of those choices and why it was not tried. Since this work began, another large effort in the 
group here has been to develop a synchronously pumped dye laser system Ref. (36, 37, 38, 39). We, therefore, explored the possibility of l'eing these extremely short (10p sec) pulses with high peak power ( $1 \mathrm{~K}$ watt when cavity dumped) to efficiently generate tunable U.V. light. This at first would seen like a very attractive system because the pulse rate is very high $(80 \mathrm{MHz}$ without cavity dumping and $4 \mathrm{MHz}$ with cavity dumping) and we could do quasi-C.W. spectroscopy with relatively high efficiency in our doubling crystal due to the high peak power. The very fast repetition rate was actually the problem with the system when we look at our method for detecting the FIR output of the device. As will be discussed in the next section, all of the FIR detectors available to us are of the thermal type, and therefore, have associated with them long ( $1 \mathrm{msec}$ ) time constants which make them unsuitable for a fast repetition rate system. They are in fact much less than ideal for the extremely fast pulses used in all of the configurations so $f$ ar discussed. We would not gain any signal to noise advantage because we could not use any temporal filtering. The best mode for the detectors used would have been a C.W. pumping system chopped at a low rate $(\sim 10-15 \mathrm{~Hz})$. As mentioned above, the appropriate pump lasers were not available.

Only a few of the many possible ways of exciting the upper state have been mentioned here, but for the purpose of the experiment the ones mentioned were most appropriate in terms of results and equipment available. 


\section{Experimental Procedure}

The experimental work began with the construction of a working heat pipe oven. As mentioned in the first section of this chapter, we began with a simple device approximately $50 \mathrm{~cm}$ long and $1.9 \mathrm{~cm}$ in diameter. With this apparatus, we had three distinct goals in mind. The first was to characterize the operation of the oven itself. The second was to do spectroscopy on the potassium vapor using white light sources as well as the laser systems men- tioned above. The last goal for this device was to observe FIR stimulated emission and observe the Stark shifted emission.

We, therefore, began by operating the heat pipe under conditions ranging in pressure from .1 Torr to 10 Torr and in temperature from $270^{\circ} \mathrm{C}$ to $405^{\circ} \mathrm{C}$. A simple but effective diagnostic for the presence of potassium vapor was the use of a flashlight. When the pipe is operating properly the transmitted light takes on a greenish tint when shined through the vapor. This is the result of very strong absorption in the red part of the spectrum due to the presence of molecular potassium at about the $1 \%$ level. Another straightforward method for observing the presence and position of the $K$ vapor was a logical extension of the above experiment. A small HeNe laser was used to probe the vapor and once again was absorbed by the $1 \%$ of molecular species which in turn fluoresced in the red and appeared as a bright red column the diameter of the HeNe laser beam and the length of the potassium vapor region. The laser beam was not absorbed completely and the column in the heat pipe appeared uniform in intensity at all 
pressures tested. At the higher pressures it was much brighter than at the lower pressures due to the higher total vapor pressure of potassium in the pipe and because at higher temperatures the percentage of molecular potassium increases. In all experimental arrangements, these two simple diagnostics proved invaluable in diagnosing prablems associated with the heat pipe itself.

The next step in the characterization of the system was the absorption spectroscofy of the potassium vapor. A variety of white light sources were used to observe the absorption in the vapor, but the broadest source with the fewest annoying features (such as spikes) turned out to be a quartz halogen lamp. The output of the lamp was put directly into the heat pipe and the output of the heat pipe was then focussed into either a Spex $3 / 4$ meter monochrometer Model 1802 or a Spex Doublemate Mode 11672 double monochrometer. The 1802 has higher resolution but the 1672 was generally more available and had enough resolution for most applications. The detectors used were generally either an RCA 1 P28 or RCA 8850 photomultipliers. Both tubes have very little response past $700 \mathrm{~nm}$ so our spectra were generally limited to the $250 \mathrm{~nm}$ to $700 \mathrm{~nm}$ range. Due to the low brightness of the 1 amp and the broadness of the detector-monochrometer system, only the strongest lines were expected to be seen. Even though our detection system was very poor above $700 \mathrm{~nm}$ the features at $760 \mathrm{~nm}$ were expected because they are the strongest lines in potassium. This feature and two others were observed. They appeared at 590 
nm and $404 \mathrm{~nm}$. The $404 \mathrm{~nm}$ line is attributed to the $4 \mathrm{~s}-5 \mathrm{p}$ transition, the $766 \mathrm{~nm}$ line to the $4 \mathrm{~s}-4 \mathrm{p}$ transition and the $590 \mathrm{~nm}$ line to the $4 p-5 d$ or $4 p-7 s$ transitions. When the lamp output was run through the monochrometer first and then into the heat pipe, only the $4 s-4 p$ and $4 s-5 p$ transitions were observed.

These observations assured us that the vapor in the heat pipe was indeed potassium and that the heat pipe was working properly. Since the detection bandwidth $\left(\sim 20 \mathrm{~cm}^{-1}\right)$ was much greater than the linewidth of the transitions involved $\left(\sim .03 \mathrm{~cm}^{-1}\right)$, we were assured that even a small detected absorption was accually very substantial.

The next step was to attempt to observe U.Y. absorption in the $4 \mathrm{~s}-13 \mathrm{p}$ transition. Using a very narrow $\left(.002 \mathrm{~nm}\right.$ or $.15 \mathrm{~cm}^{-1}$ linewidth) tunable dye laser we attempted to pump this transition for the first time. The system used consisted of a SpectraPhysics Model 171-09 argon ion laser pumping a Spectra-Physics Model 375 tunable dye laser with an optional intracavity etalon to reduce the operating linewidth. The output of this laser was then focussed with a $10 \mathrm{~cm}$ focal length lens into a KDP doubling crystal and the output of the crystal was recollimated and passed through a Corning 7-54 glass filter which passes the U.V. light but not the visible. This beam was then directed into the heat pipe by suitable mirrors and detected as it exited the heat pipe.

A set of Melles Griot ultraviolet grade neutral density filters was used to attenuate the laser beam so it would not saturate the detector. The detector used was an EG\&G model UV-4448 photodiode. The output of this photodiode was put directly onto a 
chart recorder or amplified by an operational amplifier and then put out to the chart recorder. When operating at $292 \mathrm{~nm}$ a sharp resonance was observed. Since the etalon had to be hand tuned and $\mathrm{cnly}$ had a tuning range of $1 \mathrm{~nm}$ the discovery of the absorption took several hours to accomplish. However, once tuned to the absorption, the system showed excellent stability and could be shut-off and restarted the following day and remain on the desired absorption line. Figure (13) shows a typical hand scan of the etalon. We see the laser output powers vary over the range of the scan and a sharp absorption near the middle of this range. The total scan magnitude is approximately $\mathbf{1} \mathrm{nm}$. The ultraviolet power entering the system was too small to accurately measure with the power meters available. We can see from Fig. 13 that when tuned onto the absorption, approximately $40 \%$ of the laser light was absorbed.

The neutral density filters were then removed and fluorescence from the excited states was looked for. None was observed from the output end of the pipe. The total ultravioiet power was still very low because of the poor doubling efficiency of a c.w. system. It was decided that the best possibility of observing the fluorescence was to look back at the excited atoms from the input and of the pipe. This would put the observer as clase as possible to the excited vapor. A front surfaced mirror was constructed with a small (2 mm) hole in the middle to let the U.V. into the pipe. It was placed at $45^{\circ}$ to the laser beam and an appropriate lens was used to focus the output into a $1 / 4$ meter Jobin Yvon 


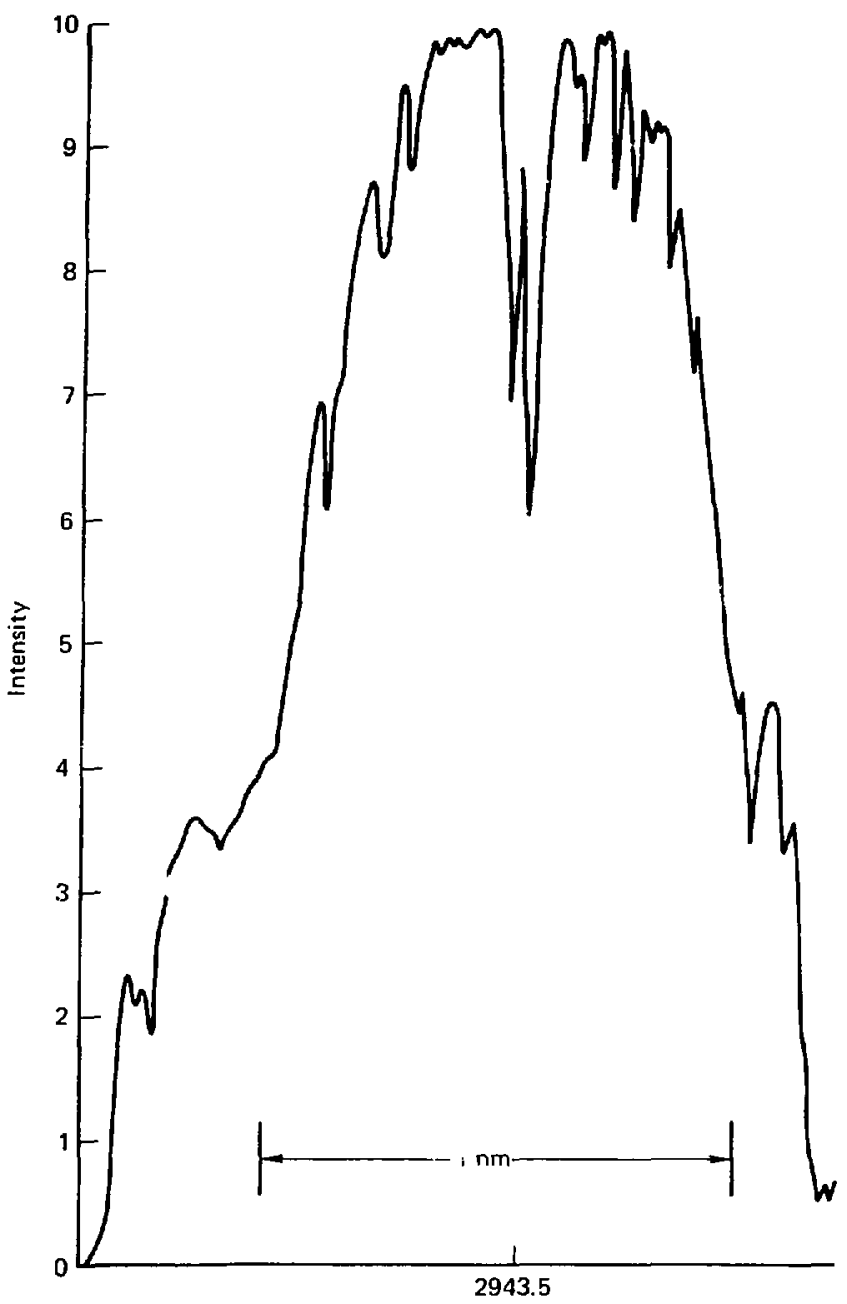

Wavelength

Figure 13. A typical etalon scan of the Spectra-Physics 375 dye laser showing the $4 s-12 p$ doublet. 
Model H2OV monochrometer. This was selected because of its very high throughput. The detector was once again an RCA 1P28 photomultiplier tube. The output of the tube was put into a Kiethley Model $417 \mathrm{~K}$ chromatagraph electrometer and the output of this was put into a strip chart recorder.

No fluorescence was shserved with this configuration either, so it was decided to pump a lower transition which would absorb more efficiently and possibly have a corresponding higher fluorescence output. The $4 s-8 p$ level was chosen because of its strength (the Einstein A coefficient for $4 \mathrm{~s}-8 \mathrm{p}$ is $\sim 10$ times greater than that for $4 s-13 p$ ) and the U.V. output of the pumping system was about the same. No visible fluorescerce was observed.

It was then decided that we should attempt to observe the FIR region of the spectrum since the first transitions out of the upper states are in this region. The apparatus was set up as shown in Figure (14). The U.V. input was chopped at $10 \mathrm{~Hz}$ and the output of the Golay detector was put into a Princeton Applied Research (PAR) Model HR-8 lock-in amplifier with a type D differential pre-amplifier plug in. The output of the heat pipe was focused into the Golay cell by a $30 \mathrm{~cm}$ focai length polyethylene lens $5 \mathrm{~cm}$ in dimeter. A black polyethylene filter was used to block all wavelengths shorter than $50 \mu \mathrm{m}$ from entering the detector. No signal could be observed with the lock-in amplifier when a time constant of 10 seconds was used. This confirmed the early suspicion that we would have to go to a pulsed system. 


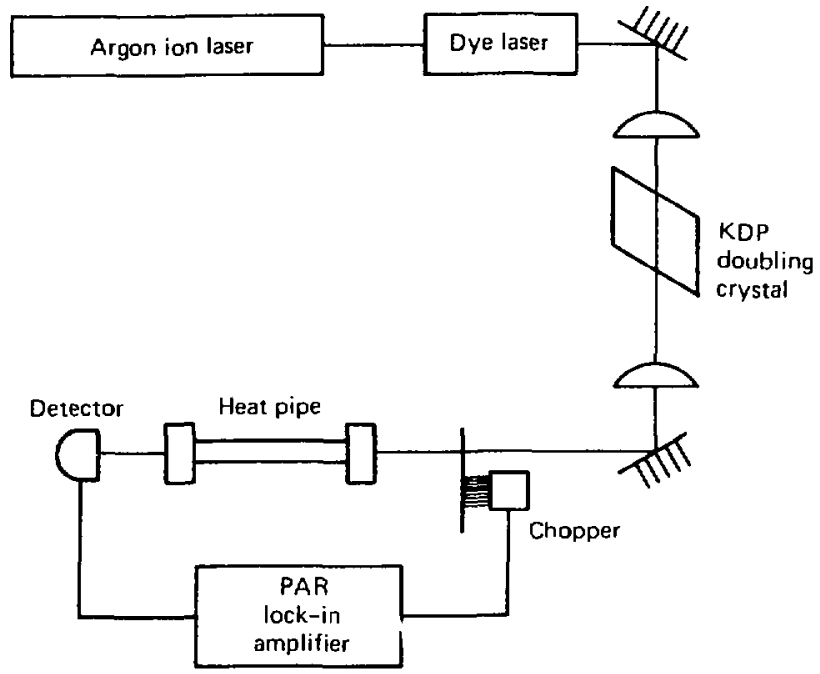

Figure 14. The configuration used for C.H. spectroscopy using the Spectra-Physics: $170 A^{+}-375$ dye laser pum?. 
At about this time, it was suggested that a useful method for finding the absorption lines in p(tassium might be obtained by using the opto-galvanic effect (40). In this configuration the laser is directed into a hollow cathode discharge tube and excites the atom to an upper state at which time it is ionized due to the high effective electron temperature. The signal is then detected as a voltage change on the electrodes of the hollow cathode tube. This is a potentially very sensitive device for locating resonances in any atomic species that can be operated in a hollow cathode tube or a discharge. In our experiments, a comerciaj hollow cathode tube, Wesı inghouse Model WL-22863A, with potassium in a neon atomsphere was used with a constant current source running at $15.5 \mathrm{~m}$ Amps. We had approximately $350 \mathrm{~mW}$ of visible power chopped at a $1.8 \mathrm{KHz}$ rate. The voltage across the electrodes was monitored through a 5 pf capacitor by the PAR lock-in amplifier with a 100 $M \Omega$ input impedence. The output of the PAR was put onto a strip chart recorder. The output is shown in Figure (15). This scan is centered at about $580 \mathrm{~nm}$, but was not well calibrated due to the need for hand tuning of the dye laser etalon. We see that there are at least three features in the scan. These would correspond quite well to the $4 p-5 d$ and $4 p-7 s$ transitions in potassium. After this initial success, we decided to try to use the same effect to locate the U.V. transitions. Unfortunately the commercial hollow cathode tube was equipped with a pyrex glass window which was unsuitable for ultraviolet. An attempt to use a demountable hollow cathode tube loaded with potassium and operated with argon 


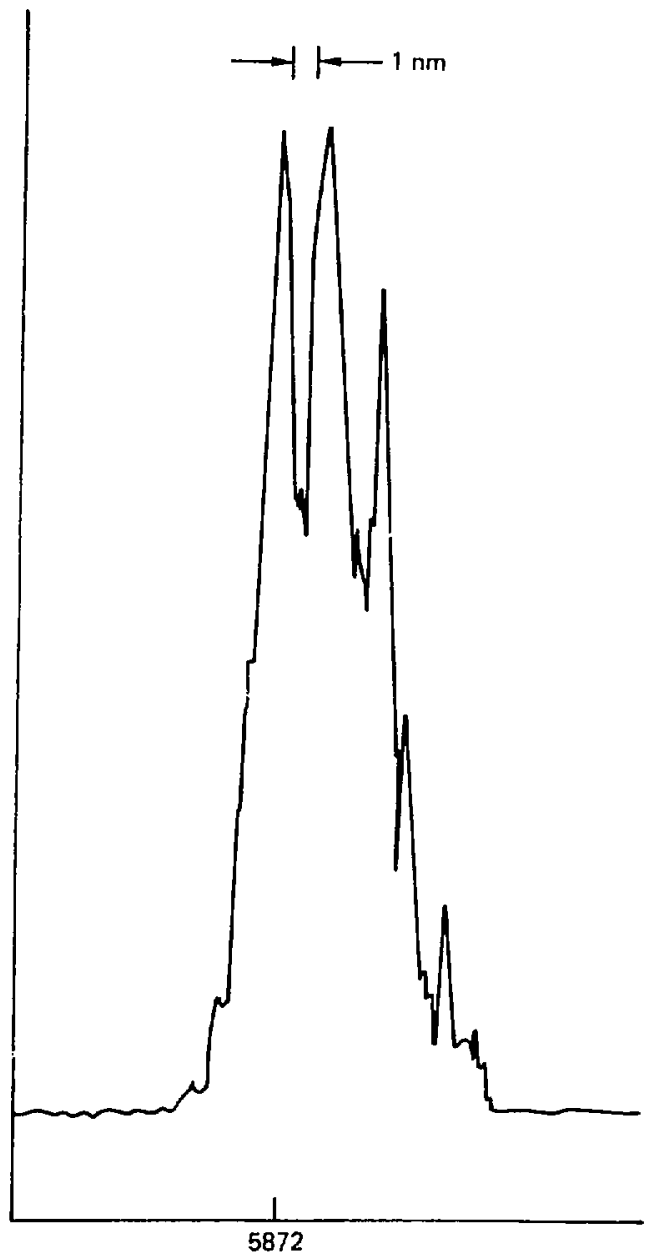

Figure 15. The opto-galvanic signal associated with the group of lines near $580 \mathrm{~nm}$. 
as the fill gas was unsuccessful. The tube, designed in the Chemistry Department at Lawrence Livermore Laboratory (41) was a much different configuration than the commercial model, so the operating parameters could not be compared directly.

We now began once again to look at the heat pipe oven for an absorption. The molectron laser system described above and shown in Figure (11) was used to look at the 4s-13p transition. Using the lock-in amplifiej, we found the absorptions at both the $4 \mathrm{~s}-13 \mathrm{p}$ transition and the $4 \mathrm{~s}-12 \mathrm{p}$ transition. The ultraviolet detector was replaced with the Golay cell. The output of the heat pipe was focused into the Golay with the polyethylene lens and filtered with black polyethylene. A signal was observed which depended critically on the tuning of the dye laser and the temperature of the heat pipe. The pressure in the heat pipe was raised from 2 Torr to 7 Torr to more closely reproduce the results of Ref. (30). With the lock-in amplifier time constant raised to $100 \mathrm{sec}$,, we achieved a signal to noise ratio of approximately 10 . It was at this point that our nitrogen laser failed.

The next step in our procedure was to try another available pumping configuration. At this point we had a krypton ion laser available as well as a Chromatex CMX-4 flashlamp pumped dye laser. It was decided to try to excite the $k$ vapor to the first excited state using the ion laser to pump a near infrared dye and then use the flashlamp pumped laser to excite these excited atoms to the desired upper state. The experiment was set up as in Figure (16). The krypton ion laser, a Spectra-physics Model 


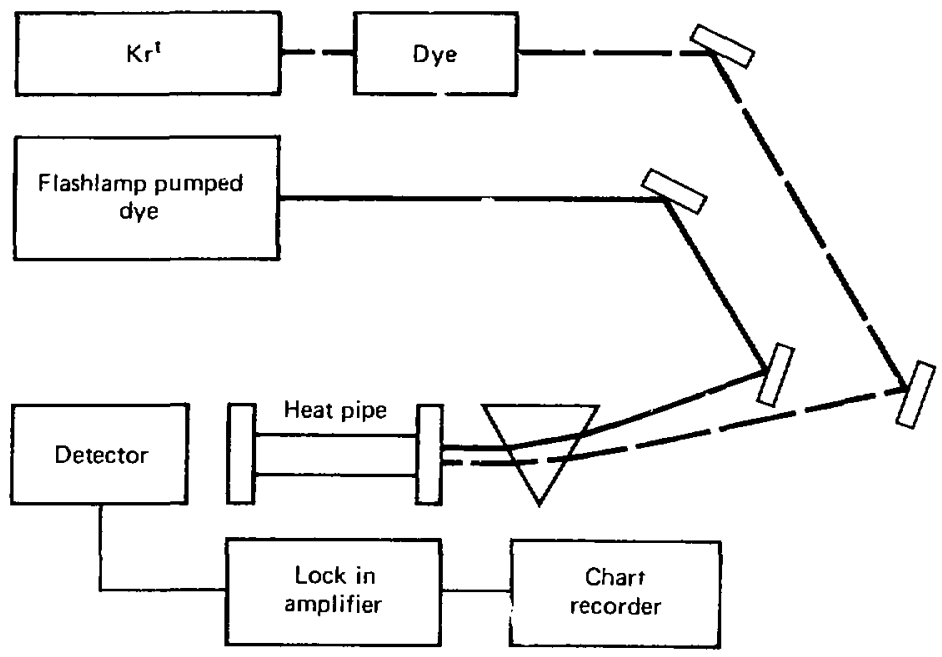

Figure 16. The configuration used for the C.H. $\mathrm{Kr}^{+}$-dye laser, CllX-4 pulsed laser experiments. 
171-09, was used to pump a Spectra-Physics Model 375 dye laser with Oxazine 725 as the working dye. The first abjective was to find the $4 \mathrm{~s}-4 \mathrm{p}$ absorption at $757 \mathrm{~nm}$ and $759 \mathrm{~nm}$. Wit's the heat pipe operative at $340^{\circ} \mathrm{C}$ and $300 \mathrm{~mW}$ of dye laser output, we saw total absorption of the near infrared laser light by the dy... Using our hole coupled input mirror at $i 5^{\circ}$, we saw very bright fluorescence in the tube in the yellow-grcen region of the visible spectrum. If all of the processes were single photon, we would not expect to see any visible light. It was also observed at thi point that the spectrum changed as we tuned the dye laser on or off of the very strong atom lines. The fluorescence did not disappear completely when thr dye laser was tuned off. It was alsa observed that the red $k_{2}$ band was very prominent when the dye laser was tuned off of the atomic lines and the intensity of this band dropped sharply when the dye laser was tuned to the atomic line.

The yellow-green features of this part of the experiment were investigated more carefully with the Spex 1672 double monochrometer. Using .25 $\mathrm{mm}$ slits on the monochrometer, we had $5 \AA$ resolution which is enough to resolve any of the atomic doublets in this region Scans were made under various pumping and heat pipe conditions and the results will be presented in the next chapter with a possible explanation for these observations.

After the short detour into the apparent multiphaton processes in the potassium vapor, it was clear that any energy lost here would not effect the FIR performance. In the process of 
recalculating the parameters for the two photon pumping scheme, however, it became obvious that the duty cycle of the blue secand photon laser would be mismatched to the first photon pump. He could saturate the first transition with the ion pumped dye laser, but the pump rate of this system could not match the pump rate of the pulsed system and we would, therefore, waste most of the second laser pulse. It was calculated that less than $.1 \%$ of the pulse would be absorbed. A brief experiment confirmed our calculations. We could see little or no absorption of the blue in the once excited vapor.

The only method available then was a pulsed single photon method. The Chromatix CMX-4 used for the pulsed part of the above experiment also has the ability to be intra-cavity doubled using various KDP crystals cut at the apprcpriate angles for phase matching at severai wavelength ranges. it also has a choice of several intra-cavity etalons available to narrow the linewidtin of the system (at the expense si tota: olyuut energy). The system was set up with $R 6 G$ as the la ing dye, the low finesse $1.5 \mathrm{~cm}^{-1}$ linewidth) etalon, and the appropriate optics to allow intracavity doubling. We obtained $.2 \mathrm{~mJ}$ per pulse output energy with a pulse duration of approximately $1 \mu \mathrm{sec}$.

The heat pipe was operated ac 1 Torr and the system was set up as in Figure (12). The laser was scanned electronically which keeps the etalon and the Lyot filter scanning at the same rate. The dye laser was scanned in the region of $292 \mathrm{~nm}$ and the desired absorption was found. Actually two absorptions were found 
approximately $10 \mathrm{~cm}^{-1}$ apart, but this was found to be a problem with the scanning dye laser. The resolution of the system was too low to permit the observation of the doublet $13 p 1 / 2$ and $13 p 3 / 2$ states. The Golay cell was then positioned at the output of the heat pipe and a black polyethylene filter was used to filter out all radiation with wavelengths shorter than $50 \mu \mathrm{M}$. Using the PAR lock-in amplifier a signal was observed out of the Golay cell. The signal depended on the tuning of the dye laser and the temperature of the heat pipe, but was very weak. Several focusing arrangements were tried, but the expected output in the FIR was never achieved. The pressure was raised to 5 Torr and the experiments with focusing and tuning repeated with negative results. The energy was being absorbed, but was not coming out in stimulated emission along the axis of the pipe.

It appeared as if the cavity needed to be defined better to eliminate the possibility of stimulated emission off-axis. Mirrors were installed in the heat pipe on flexible stainless-steel bellows. They were hole-coupled polished stainless steel ( $\lambda / 4$ at optical wavelengths) mirrors with a $1 \mathrm{~mm}$ input hole and $1.5 \mathrm{~mm}$ output hole. The input mirror had a $1.5 \mathrm{M}$ radius. These were aligned optically using a He Ne laser. The absorption spectroscopy was done with a PAR Model 162 boxcar integrator, using two channels and a ratio function. Both detectors were again EG\&G UV-444B photodiodes. The beam was split at the input with a piece of $z$-cut quartz and directed into one of the photodiodes as a reference. The main beam entered the heat pipe through a sapphire 
window and exited through a polyethylene window. Because polyethylene does not pass ultraviolet light well, the detector accually looked at the fluorescence of the polyethylene due to the U.V. light. Care was taken to eliminate any detector saturation problems. Absorption was found quite easily, but the FIR was not observed.

During the period several series of experiments were run to try to optimize the FIR output. The system was run from .7 Torr to 8 Torr with no FIR output observed. Focusing was also varied from $25 \mathrm{~cm}$ to an essentially collimated beam, again with negative results. It appeared now that a fundamental problem was showiny itself. Since all other variables had been changed without success, we decided to return to the short ( $5 \mathrm{nsec}$ ) pulse system and try to recover the FIR signal. Beam time on a Molectron nitrogen pumped dye laser system was arranged and the apparatus was set up. The dye output of the laser was $36 \mathrm{KW}$ peak which converts to about $290 \mu \mathrm{J}$ per pulse. This was then doubled with the characteristic $4 \%$ to give approximately $11.5 \mu \mathrm{J}$ per pulse in the U.V. Using the boxcar to detect the absorption, we scanned the dye laser. The absorption was found to be equivalent to an optical density of .23 and very narrow. This optical density may be related to the intensities by,

$$
0.0 .=\log _{10}\left(\frac{I_{j}}{I_{t}}\right)
$$

where $I_{i}$ is the intensity of the incident light and $I_{t}$ is the intensity of the transmitted light. 
Once again the Golay cell was set up and the appropriate filters were used. The lock-in amplifier was connected and used with a 100 sec time, constant. A strong signal was observed. With a time constant of 30 seconds on the lock-in, a signal to noise of $6: 1$ was observed. The laser was then tuned off of the resonance by $.04 \mathrm{~nm}$ and the signal went away completely. When the laser was brought back to the same place, the signal returned. This was done several times on both sides (higher and lower frequency) of the atomic resonance. The z-cut quartz beam splitter was then removed and the signal increased greater than 10\%. With a 10 second time constant the signal to noise was $3: 1$. Being able to reduce the time constant was very important because the laser parameters could be adjusted in a reasonable amount of time. The Corning 7-54 filter was removed to let all of the visible into the heat pipe as well as remove any attenuation of the U.V. There was no apparkint effect on the FIR power measured. The insertion and removal of the beam splitter appeared to make a $30 \%$ difference in FIR power measured at this point. The difference between this measurement and the earlier one, could have been due to different insertion angles. The earlier ones were done with the disk inserted closer to Brewster's angle. At this time, several things were done to try to improve the output of the device. The position of the device was changed with respect to the focal point of the beam and no change was apparent. The output was focused with several different polyethylene lenses, but again no improvement was seen. An estimate of the power out in 
the FIR was made. The lock-in indicated a signal of $1 \times 10^{-4}$ volts out of the Golay. The responsivity of the detector is $1.5 \times$ $10^{6}$ volts/watt so we apparently have $6.67 \times 10^{-11}$ watts average or $1.3 \times 10^{-3}$ watis peak for a pulse duration of 5 nsec and a repetition rate of $10 \mathrm{~Hz}$. This means about 6.5 picojoules per pulse were being generated.

The dye laser, as mentioned earlier has a linewidth of .5 $\mathrm{cm}^{-1}$. In the last chapter we saw that the absorption linewidth for the potassium should be ibout $.033 \mathrm{~cm}^{-1}$. We, therefore, tried to narrow the dye laser li newidth to $.1 \mathrm{~cm}^{-1}$ using an intracavity etalon. The insertion of this dropped the output intensity of the dye by $70 \%$ and the U.V. output even further (due to the $I^{2}$ dependent efficiency of the doubling crystal). The FIR output was not changed much although the apparent optical density of the vapor increased dramatically. The stability of the laser suffered markedly from the insertion of the etalon so we removed it and did all subsequent experiments with the laser operating with a $.5 \mathrm{~cm}^{-1}$ linewidth.

With some degree of confidence in our pump method, we decided to continue on to the next step of the experiment. Testing the first Stark cell heat pipe oven began. The configuration of the pipe was quite different from that of the normal pipe and the operating characteristics needed to be checked. The mirrors were installed on the pipe at the outset of the experiments because of the added difficulty of working on this system in a glove box. This addition made broad band laser absorption spectroscopy 
difficult due to the beam movement during scanning. Vapor was successfully generated in the pipe and the U.V. laser absorption was located. The FIR output was found, but the potassium reservoir ran dry quite rapidly. This proved to be a fundamental problem with the design of the pipe. The ratio of the diameter of the central "can" to its height was too large to allow sufficient cooling near the base of the can. This in turid allowed much of the potassium to reach the cold region where it solidified. The lack of wicking also proved to be a major problem for the return of liquid potassium to the reservoir area. Several modifications of this device were tried, but it was concluded that nothing short of a complete redesign would be suitable for the experiments envisioned. This was not undertaken because the second design was already in the process of being built.

At about this time it also became obvious that a dedicated pump laser system to provide the tunable U.V. laser light wuld be needed. A suitable system was fo $-d$ ir the Las.. Isntope Separation program at $L_{i}:=:$. l $^{i-}$. gialned to Use the system in our laboratory for an ex+ended period of time. The system was a Molectron U.V. 1000 nitrogen laser, [ L-400 Dye Laser system identical to the one previously used except for the welcome addition of a stepper motor scan option which allowed .001 nm scan resolution and direct wavelength readout. This was very useful in scanning the dye laser output over a small range and then being able to return to the exact spot of the desired feature. Since the doubling crystal is manually tuned for phase matching, the 
scan lumit for the U.V. is about $1 \mathrm{~nm}$ before the crystal hids to be retuned. This did not prove to be a problem. The output of this dye laser was found to be calibrated extremely well with the wavelength dial on the chassis. This proved to be quite convenient when looking at different levels in the potassium. The output power of the nitrogen laser also appeared to be quite good which allowed us to run it at a lower voltage to maintain stability.

The first set of experiments with this laser system were to find the FIR with our conventional heat pipe oven. All mirrors were removed as they had shown no advantage on the system previously and had made absorption spectroscop̣ difficult. They were not used again. The heat pipe was set up as in Figure (11) with the boxcar to detect the absorption. The $4 \mathrm{~s}-13 p$ absorption was found very easily in the heat pipe running at 1 Torr. The FIR signal was found immediately with an increase in signal level over previous measurements of a factor of 5 . Tne output of the Golay cell was again put into the lock-in with a 1 second time constant and a signal to noise ratio of 5 was achieved. This allowed us to adjust optics directly as we watched the lock-in to peak up the signal. It was clear, however, that better signal to noise ratio would be needed if we were to go through a wavelength measuring device and still be able to be detected. To solve this problem, two potentially useful bolometers which are suitable for the submillimeter part of the spectrum were located. The first is a Molectron Model SB15-202(SL) Silicon Bolometer Cryostat System, 
which consists of a Model DB2-1000 silicon bolometer, a Model DW2O liquid helium dewer and a Model AB-10 pre-amplifier. This system was cooled several times, but was never working satisfactorily. The pre-amplifier seemed to be the problem area for the system, but the exact cause of the problem was never fully elucidated. The second bolometer system used was a Texas Instruments Model 170866 Bolometer system. This system consisted of a galium doped germanium (Ga:Ge) bolameter, a Texas Instrument Mode] CLF 3 cryoflask, and a preamplifier. This system was particularly well suited to the task because it covered the region of the submillimeter spectrum of interest $\left(20-200 \mathrm{~cm}^{-1}\right)$ and had very little response outside this region. This is partly due to the response of the detector itself and partly because of the cooled sapphire and black polyethylene filters which cut off all higher frequencies.

The bolometer was cooled to $4.2^{\circ} \mathrm{K}$ with liquid helium and the output of the pre-amplifier was put into the lock-in amplifier: Although the output level on the lock-in was about the same as that of the Golay cell, the signal to noise was much better. The pre-amplifier could be used in two modes. One was with a variable bias control and the second was with a fixed bias set according to factory supplied calibration charts. In the first mode the signal to noise was 10:1 with the current reading $1.5 \mu \mathrm{A}$ on the pre-amplifier. This is to be compared to the $5: 1$ signal to noise output of the Golay cell under identical conditions. In the second mode, with the internal bias control, the 
current read $8.2 \mu A$ and the observed signal to noise was 40:1 with a 1 sec. time constant.

To illustrate the relative performance of these detectors several scans were made of the U.V. pump laser while monitoring the FIR output of this heat pipe. These are shown in Figure (17). We can see from this the dramatic decrease in noise obtained by using the bolometer. This advantage is somewhat offset by the increased difficulty of using the bolometer and by the limited bandwidth of the device for some measurements in other wavelength regions.

At this point it was clear the signal to noise ratio was good enough to attempt a wavelength measurement, but the bean characteristics were uncertain. If we hoped to see the radiation at a. on the other side of a wavelength measuring device the beam's divergence would have to be controlled. To do this several different polyethylene lens were tried and it was found that the beam could be controlled quite easily by simple optical techniques. For instance we found that a $30 \mathrm{~cm}$ focal it ith lens focused the beam sufficiently that there was little or no signal loss when the bolometer was placed $31 \mathrm{~cm}$ from the lens. The focusing of the pump beam had very little effect on the FIR output so the pump beam was collimated to obtain the best possible output beam quality. The FIR output was very well defined along the axis of the U.V. pump beam as evidenced by the sharp dependency of the FIR signal to the spatial position of the detector. 


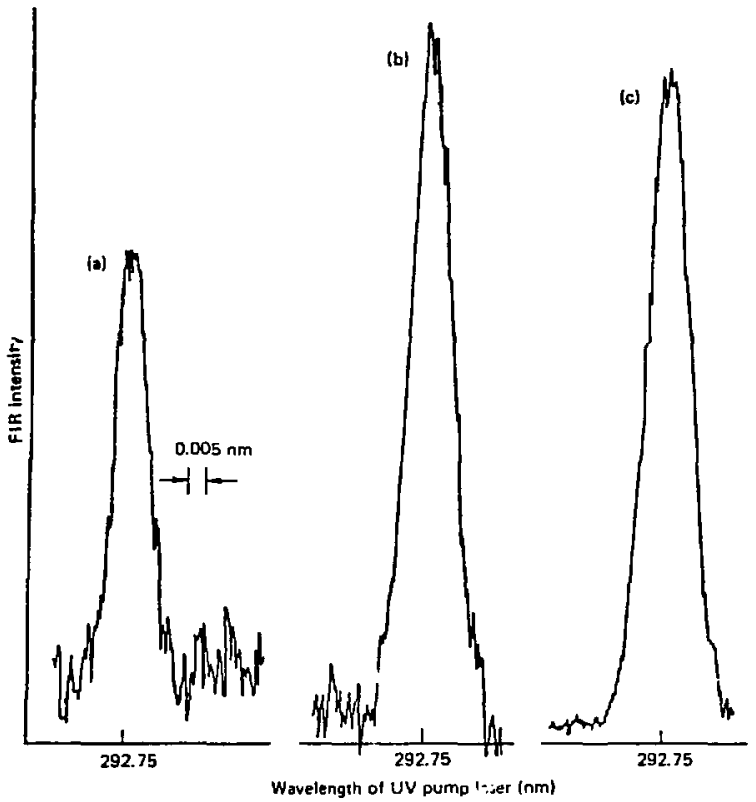

Figure 17. $F_{\alpha} R$ output from the detectors as the pump ?aser is scanned.
a) the golay cell
b) the Ga:Ge bolometer with $<2 \mu$ A current,
c) the $\mathrm{Ga}: \mathrm{Ge}$ bolometer with $8.2 \mu \mathrm{A}$ current. 
Our first attempt to measure the wavelength of the FIR signal was simulated by the use of microwaves. A Fabry-Perot interferometer was constructed using one 6 inch diameter front surfaced flat mirror and one 6 inch diameter one meter focal length front surfaced mirror. The microwave power was injected with a beam splitter and the circulating power was monitored near the edge of the curved mirror. The microwave frequencies could be measured to about 10 megahertz. Using a modulated microwave source (1:" sec puises at $10 \mathrm{~Hz}$ ) we simulated the FIR system ssed the Golay as our istor. Using the lock-in amplifier we detected the microwave igignal at the one milliwatt peak level. This was, however, a $1 \mu$ second pulse, and therefore, much more energy than would be present in the FIR. The FIR was then injected i,ito the uvity with negative results. It is postulated inat cavity losses are greater for the FIR and as mentioned above, energy available is considerably less.

It was therefore decided to go to a high throughput system such as a Michelson interferometer. The advantages of this system are low losses, since it is a single pass system, high finesse, and ease of construction. The interferometer was constructed from two flat, front surface, 6 inch mirrors mounted on standard NRC mirror mounts and moved by Ardel-Kinematic translation stages wit:. fine translation controls. Translation resolution was $1 \mu \mathrm{m}$ and total travel was $2.5 \mathrm{~cm}$. The beam splitter was the most critical comporient of the system and many materials were considered. Mylar was the material of choice and $12.5 \mu \mathrm{M}$ mylar was selected as the 
correct thickness for this wavelength range (42). One mirror remained stationary and the other was scanned by either a synchronous motor or a stepper motor. Both scan methods provided satisfactory results. The system was aligned by removing the heat pipe from the dye laser beam path and allowing the visible dye output go through trie interferometer. The mirrors were aiigned by observing the interference pattern of the laser on the output polyethylene lens. The system was then scanned and cherked to insure good alignment throughout the scan. The heat pipf. was then placed back into the beam path and the throughput of the interferometer was measured. The system routinely passed $20 \%$ of the total input power when a polyethylene lens is used to focus the FIR into the interferometer. The beamsplitter was enlarged and the mylar stretched over a pciystyrene frame to give a good optical surface and prevent any aperturing due to the beamsplitter frame. Initial spectra were then run using the Golay celi as the detector. The initial results were very encouraging. A definite interferometric patcern was seen. Another problem that developed rather suddenly was the damage done to the instrument by an earthquake. The detector which had been sitting on the laser table, fell off and damaged the helium fill tube. The Molectron dewer was available however and were able to move the Ga:Ge bolometer to this dewar and still use it. A good signal to noise was obtained with this system but we still had the problem of data aquisition and reduction. 
At this point we decided to incorporate a digital computer into the apparatus to take, analyze, and display the data. In this respect we were fortunate to have available a Tektronix 4051 graphic computer system for a dedicated application. Although the system had been used in conjunction with experiments befare, there was no applicable software available. The project became, thereiore, to interface this system to our existing instrument. The system was quite straightforward to work with and the necessary program was written quickly to take the readings off of a digital voltmeter attacheo to the output of the lock-in amplifier. The data was then normalized to eliminate a d.c. offset due to poor modulation in the interferometer. This was a minor problem steming from the less than perfect mirror mounting system used which created misalignment during a scan. The data were now ready for reduction by Fourier transform techniques.

The Tektronix computer system proved quite valuable at this point by having available a hard wired ROM (read only : :emory) pack with a Fast Fourier Transform (FFT) program, a Taper program to provide a Hanning function, and a rectangular to polar conversion to go from the real and imaginary output of the FFT program to an amplitude and phase output which was much more useful for our purposes (43). The Taper program was the first used in our data reduction process. This function compensated for what is sometimes termed "leakage." Leakage occurs when we acquire periodic data which is not an integral number of cycles and transform this data. This generates broadening and attenuation of the spectral 
lines due to the violation of some assumptions that are made in the FFT program. To alleviate this problem we multiply the time domain waveturm by a window function to remove the discontinuities in the data. The function used here is the Hanning function and is given by

$$
x(t)=\frac{1}{2}-\frac{1}{2} \cos \frac{2 \pi t}{T_{c}}, \quad 0 \leq t \leq T_{c}
$$

where $T_{c}$ is the total scan time. This is the form of the function for $50 \%$ taper or a true Hanning function.. We also have available, with this program, the option of reducing the taper to as little as $10 \%$ or eliminating it all together. Figure (18) shows the taper function for $10 \%, 25 \%$, and $50 \%$ tapering. In our data reduction, a taper function of $20 \%$ was found to reduce leakage quite well. This amount of "windowing" reduced the artificially induced frequency components sufficiently so they were in the real noise level of the system.

The Fourier transform is done using a fast Fourier transform algorithm. Since the data is discrete we can replace the usual Fourier integral with a discrete sum. It will be sufficient here to say that the method used is the Sandy-Tukey decimation-infrequency FFT algorithm with a quarter-period negative sine table for generating the necessary complex exponentials. Ine sine wave is sampled densely enough to perform a riscrete Fourier transform on a real data array of any power of 2 between 16 and 1024. In our case the upper limit of this program was always used and 


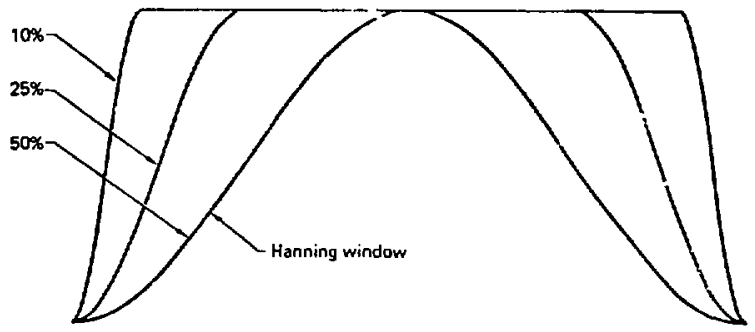

Figure 18. The window function used to "taper" the data. The $50 \%$ function is a true "Hanning" function.(43) 
represented the limit of the system based on the scan distance of the mirrors.

The next step in the data reduction process was to do a rectangular to polar coordinate transformation to display the data in a more useful fashion. This, again was done by the ROM pack using a simple access command. The resulting data arrays represented the amplitude of the data and the phase of the data. For our purposes only the amplitude information was of interest. The rest of the program written was devoted to storing this information and plotting it out in a useful calibrated fashion. A copy of the complete program listing is given in Appendix A.

The data acquisition and reduction system were now complete and we proceeded once again with the problem of measuring the wavelength of the FIR output. The lock-in amplifier time constant was set at $3 \mathrm{sec}$. to duplicate the time between data points. This has been found to be the best method for digital interferometric work using a continuously scanning interferometer (p.49 of Ref. 42). The results of these scans will be discussed in detail in the next section, but it will be sufficient to say that they were quite impressive in their ease of generation and in the consistent agreement with the calculated energy levels of the excited potassium atoms.

During the process of taking these spectra it became necessary to know the true response curve of our detector. To check this a very simple experiment was done. A hot wire source with a block body temperature of about $2000^{\circ} \mathrm{C}$ was setup. It was 
assumed that in the region of interest it would be a good approximation of a flat source of radiation. This was then chopped at a $10 \mathrm{~Hz}$ rate and put through a .5 meter aluminum tube whose output was focused into the interferometer. The output of a scan is shown in Figure (19). At first it appeared that we had excessive noise in our system so it was run again with identical results. After some reflection we decided it must be water vapor so the absorption peaks were compared to the well dorumented water vapor spectrum in this region. All of the absorption found could be identified as water vapor lines and none of the expected features were missing. From these results it was clear that the whole interferometer had to be enclosed in a water free environment. This was done by placing the device in a polyethylene glove bag and using a small positive flow of argon gas as well as bottles of desicant. The experiment to verify the absence of water vapor features was not completed. The response of the $\mathrm{Ga}: \mathrm{Ge}$ bolometer and filter system has been approximated by the curve drawn over the water vapor spectrum in Figure (19).

The next major step in the experimental process was to generate the FIR in an environment which would allow Stark tuning. As mentioned earlier the first design for a heat pipe with Stark plates was unsuccessful in producing FIR output. The second design, described in detail earlier, was now ready to be tested. Before loading the device, it was tested for field breakdown with only argon gas present. The breakdown was observed through the side window while a d.c. voltage was increased. This was done at 


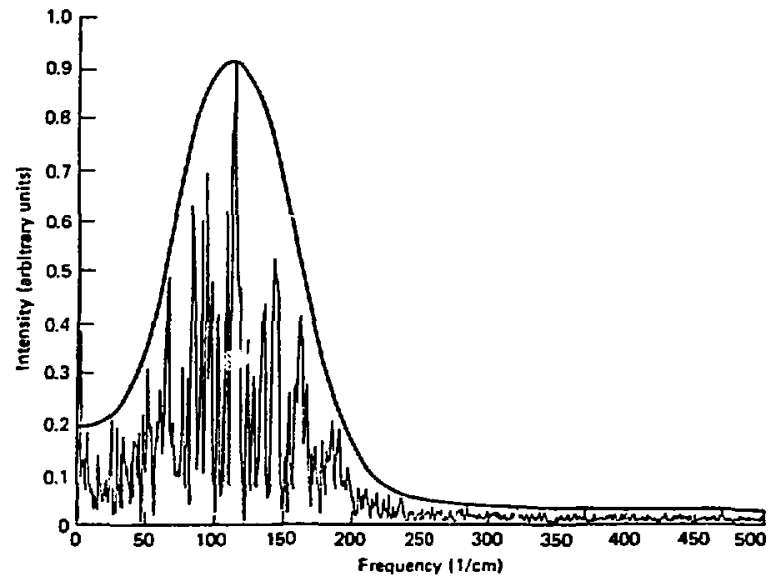

Figure 19. The bolometer response to a broadband source (hot wire) showing the water spectrum due to atmospheric water. 
pressures ranging from .1 Torr to 10 Torr and it became clear that the minimum of the Paschen curve for this system was at about 1 Torr. This meant that the breakdown would occur most easily (with the smallest applied voltage) at 1 Torr. This was quite unfortunate because all work to date had indicated that 1 Torr was a very convenient pressure and produced very good quality fIR emissions. It was, however, found that the breakdown could be controlled quite well at a pressure of .3 Torr. Even here we could only obtain a c.w. field of $800 \mathrm{~V} / \mathrm{cm}$. This was not going to be enough to shift the levels so their relative difference would be measurable. At this point it was suggested that a pulsed field would be ideal in this application. If the stimulated emission occui's in a very short time after the initial exciting pulse, then we need only have the field on a short time to shift the levels of interest. Because the rate of arc propagation in gases at these pressures is about $l_{\mu} \mathrm{sec} / \mathrm{cm}$, we could reduce or eliminate the problem by keeping the field on for $1 \mu$ sec or less. (44) To do this, a pulse generator was constructed which could deliver pulses of up to $2500 V$ with widths variable between $900 \mathrm{nsec}$ and $3 \mu \mathrm{sec}$. This design is shown in Figure (20). It was found that a pressure of .3 Torr was necessary to be able to hold off the 2000 volts to be used. The field could be held off with no sign of arcing until the pulse width was increased to $2 \mu \mathrm{sec}$ at which time the onset of ionization became apparent in the heat pipe. The pulser was triggered by the laser trigger and the pulse turn on and turn off were separately variable so we could observe the laser pulse with 


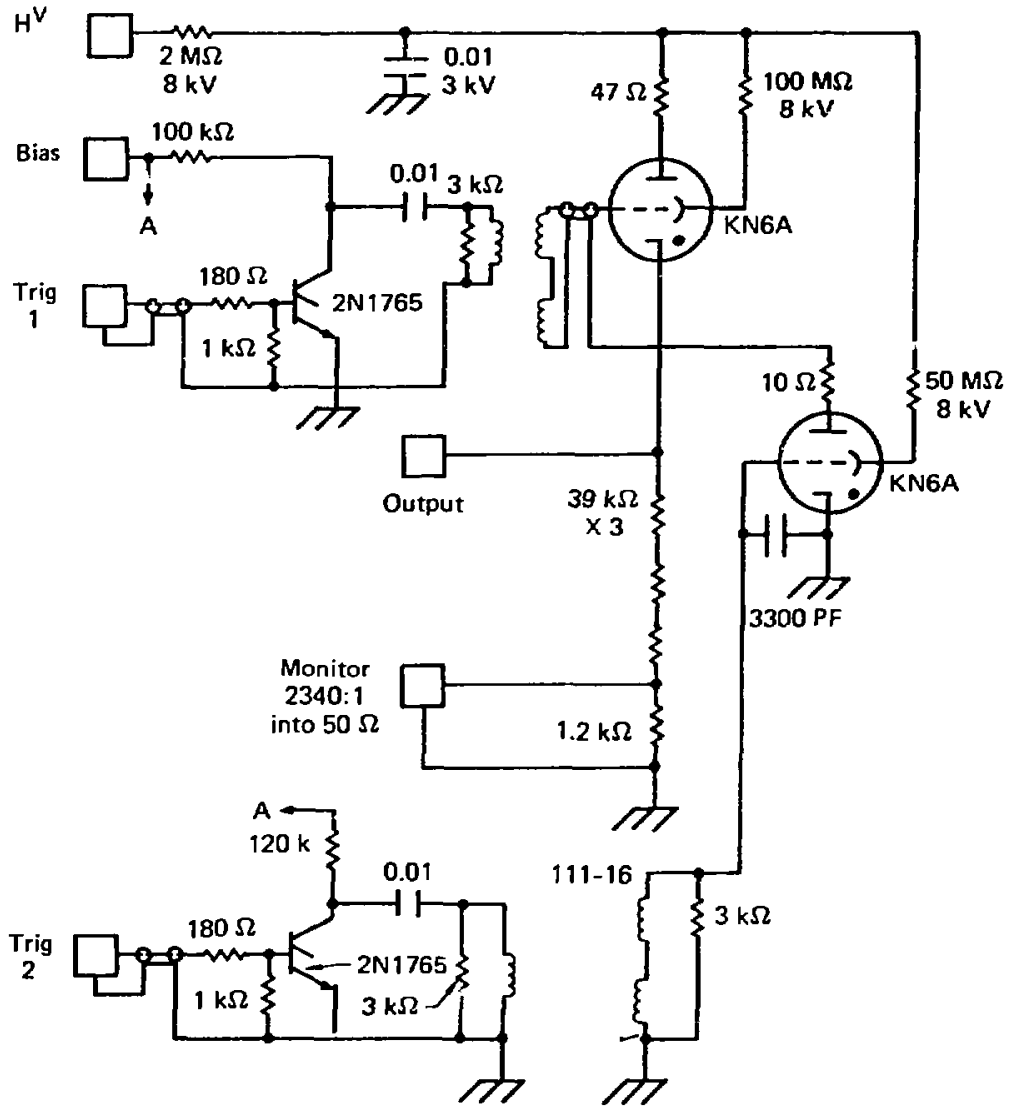

Figure 20 . The schematic of the high voltage pulse generator. 
a fast detector (Molectron P305 pyroelectric) and position the electric field pulse to start $100 \mathrm{nsec}$ before the laser light pulse arrived.

The heat pipe was then loaded with 3 grams of potassium metal and a vacuum pumped on it for 24 hours to get rid of all residual solvents. The pipe was backfilled with 1 Torr of argon and the pipe heated to $100^{\circ} \mathrm{C}$ to release any irapped gases in the metal sticks. A vacuum was again pumped on the system and the rate of rise of the pressure in the system was checked. This procedure was repeated until the system held a yacuum $(<\mathrm{l} \mu$ of $\mathrm{Hg})$ well. The system was then filled to the desired working pressure with argon and the heaters were turned up to the appropriate temperature. As mentioned earlier, this heat pipe was particularly simple to align optically because of the long window in one side. The visible light from the laser was allowed to enter the heat pipe which was aligned to let the light exit through the $Z$-cut quartz window. The Michelson interferometer was aligned with this output and the detector put into place.

The pump laser was already tuned to the correct wavelengit from the last set of experiments with the old heat pipe, so we just had to place the visible-blocking filter in and turn it on to test the new heat pipe. Because of the long window in the side, the fluorescent output of the device could be observed quite easily. The fluorescence was clearly visible with no laser tuning necessary and the pump parameters such as doubling crystal angle and laser cavity adjustment could be made while monitoring the 
visible fluorescence to find the optimum conditions. The FIR was immediately found and the heat pipe was rotated to allow the FIR to exit through the polyethylene window which is better suited to this wavelength range. It became obvious at this point, however, that we would have only limited working time available on this device due to the formation of condensed potassium metal droplets on both the top and bottom screens at the interface of the cold and hot regions in the pipe.

The detection system was set up and spectra were taken of the FIR with the field on at a level of 2000 volts and with the field off. Three spectra of each were taken in an alternating fashion before the beam path of the laser was completely blocked by the growth of the droplets. The data was reduced and the three spectra with the field were averaged as were the three without the fielf. The results will be discussed in the next section. The complete system is shown in schematic form in Figure (21).

With this phase of the experimental work done, the only remaining experiments were those to clarify the deexcitation paths from the excited states. All transitions in the FIR region could be observed by the Michelson system, but we had no time ressilution due to the very long ( $1 \mathrm{msec}$ ) time constants associated with the FIR detectors. The only detectors available with the speed necessary to resolve the times expected were in the visible region of the spectrum. This was not a problen because as mentioned earlier would use the visible fluorescence to tune up the sys tem. 


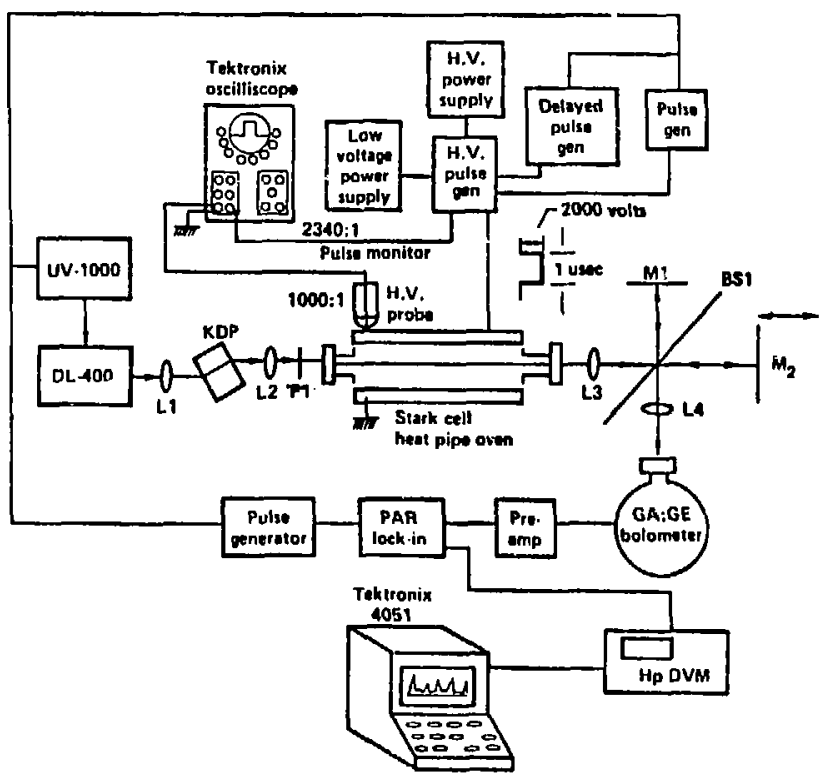

Figure 21. A schematic diagram of the total FIR experimental apparatus, 
To accomplish this part of the project we once again set up the standard heat pipe oven and set the operating parameters at 1 Torr and $330^{\circ} \mathrm{C}$. The u.v. pump light was coliimated and the visible pump laser light was filtered out by the Corning 7-54 filter. A sapphire outpu: window was used on the heat pipe to let all visible and u.v. light exit. The Spex 1672 monocrometer was used with micrometer :diustable slits to provide resolution to $1 \mathrm{~A}$ for $.05 \mathrm{~mm}$ siit width. The output of the monochrometer was monitored by a cooled ICA 31034 GaAs photomultiplier tube. This gives relatively flat response from $300 \mathrm{~nm}$ to $800 \mathrm{~nm}$. The monochrometer was aligned along the axis of the heat fipe. The li.v. pump laser light was filtered out by two techniques. First a mirror coated for $>99 \%$ reflectivity at $300 \mathrm{~nm} \pm 10 \mathrm{~nm}$ was inserted at a $45^{\circ}$ angle to deflect most of the u.v.. The rest was absorbed by the $10 \mathrm{~cm}$ focal length lense used to focus the visible fluorescence into the monochrometer. The visible transmittance of the mirror is shown in Figure (22). The lense was standard glass and transmitted none of the u.v., but did transmit well above $350 \mathrm{~nm}$. The output of the photomultiplier tube was put into the boxcar integrater and the narruw window and variable delay features of the boxcar were used to temporally select only those components of the spectrun that were shortlived $(t<50 \mathrm{nsec})$. The resulting spectra will be discussed in the next chapter. Scans were made over the region of $400 \mathrm{~nm}$ to $600 \mathrm{~nm}$ and from $400 \mathrm{~nm}$ to $800 \mathrm{~nm}$. Once again the volume of data being obtained suggested the use of the computer to store and manipulate this data so it could be 


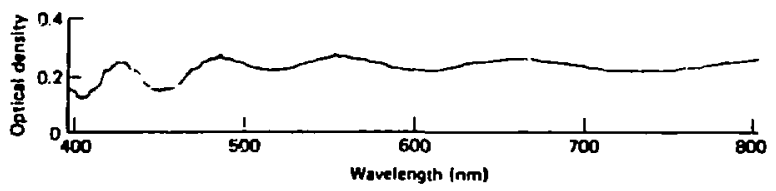

Figure 22. The absorption spectrum of the U.V. highly reflecting mirror $R>99 \%$ at $300 \mathrm{~nm}$. 
evaluated effectively. A program was written to take this data and store it on magnetic tape so it could later be compared to other scans. The program is listed in Appendix 8 . This allowed us to run scarr; with the heat pipe un and then subtract the background with the heat pipe turned off. He effectively were doing a two beam experiment with a single beam system. From these experiments we had good spectral information on the system, but still no temporal information. To achieve good temporal resolution we took the output of the photomultiplier tube and terminated it into a $1 \mathrm{k} \Omega$ load. The time response of the syste:; was then less than $10 \mathrm{nsec}$ which allowed viewing all visible fluorescence in a pump pulse limited situation. Because of the low responsivity of the photomultiplier with a $1 \mathrm{~K} \Omega$ load, we had to reduce the resolution of the monochrometer to increase its throughput. The results of these measurements will be discussed in the next section.

The last experiments done on the system ware to quantify the energy absorbed in the vapor as a function of pressure. A scieritech Model 362 power meter was used to calibrate a Molectron Miodel J305 joulemeter which was lised tc make all energy measurements. The J305 was found to have a responsivity of 3259 volts/Joule when terminated into a $1 M \Omega$ load. The data listed in Table (7) shows the results of these mexurements at various pressures. In Figure (23), we have the data displayed and a least squares fit to these data using the function 
TABLE 7

$\begin{array}{cc}\text { Pressure (Torr) } & \text { Intensity } I_{0} \\ 0.295 & 0.86 \\ 0.32 & 0.83 \\ 0.51 & 0.82 \\ 0.71 & 0.82 \\ 1.00 & 0.8 i \\ 3.00 & 0.6 ! \\ 5.00 & 0.53\end{array}$




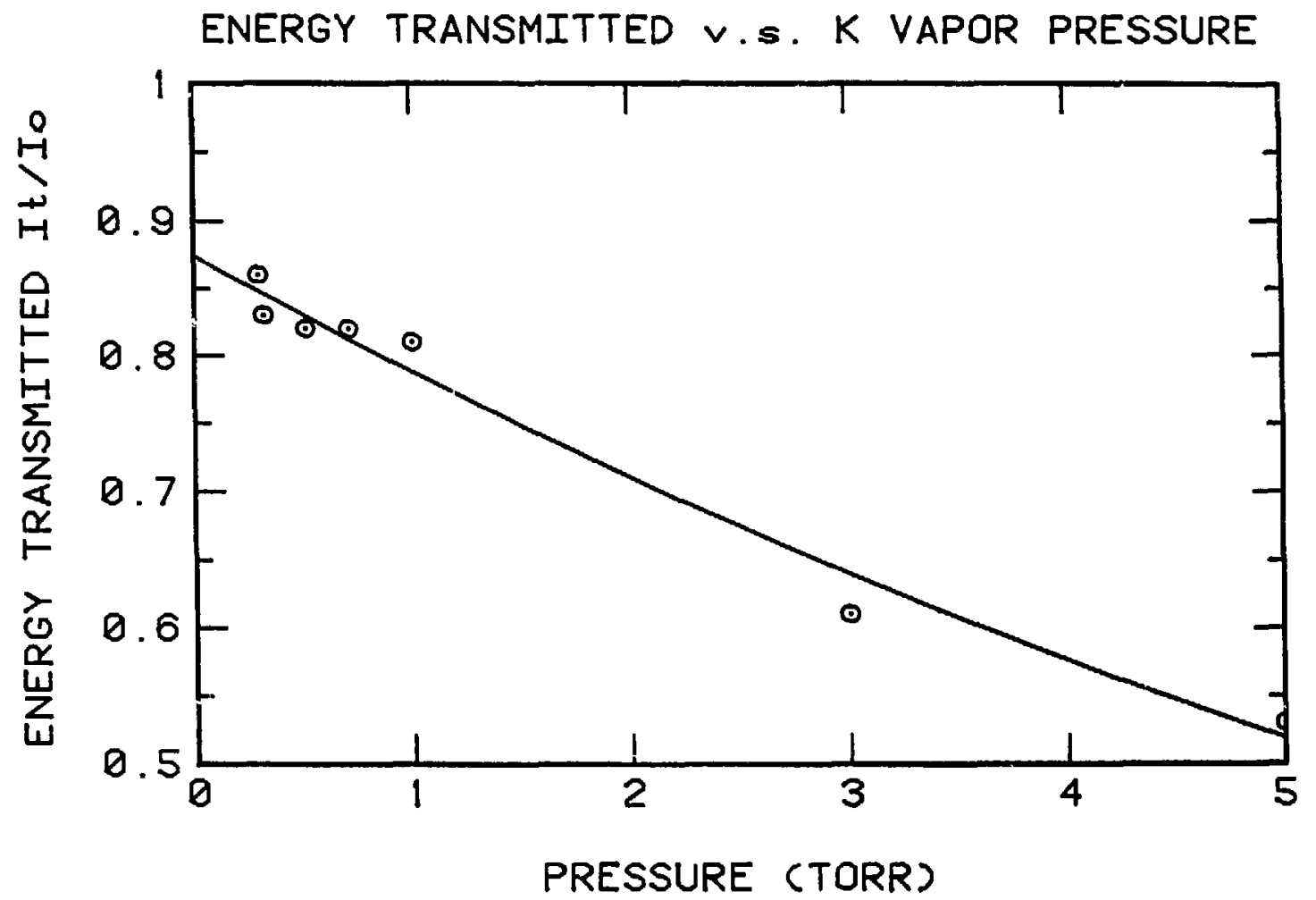

Figure 23. The absorption of the pump laser for the $4 s-13 p$ transition with an exponential 


$$
Y=A \exp (B X)
$$

The R-square error is 0.980 , the residual error is 0.000 , and the maximum residual is 0.029 .

It is difficult to determine from these results what the limit on the system is. Because of the mismatch in linewidths between the laser pump and the potassium absorption, we can say little more about the system. Needless to say, we could improve the energy coupling efficiency by using a narrower pump laser, but as mentioned earlier, this generally leads to lower energy output of the pump laser and is, not necessarily advantageous. 


\section{RESULTS}

This chapter will deal with the results of the experiments discussed in the last chapter. These results will be presented in two major sections: results from visible spectroscopy and results from FIR spectroscopy. Inciuded in the visible results will be the optogalvanic spectroscopy done using a potassium hollowcathode lamp.

We begin this chapter by discussing the results of some spectroscopy done on the potassium vapor when it was excited with a high average power continuous wave, tunable dye laser. The pumping system consisted of Spectra-Physics krypton ion laser operating at 3 watts on the $647 \mathrm{~nm}$ line pumping a Spectra-Physics dye laser using Oxizine 725 as the dye. This system was capable of zroducing 1 watt of tunable laser power in the regi:n of the $767 \mathrm{~nm}$ $4 s-4 p$ fundamental line in potassium. The fluorescence in the vapor was observed by a front surface, hole coupled mirror set at the input end of the heat pipe at a $45^{\circ}$ angle. This allowed us to view the input boundary layer of the potassium as it was being excited. Visual inspection revealed components of fluorescence in the green-yellow portion of the spectrum. These were studied more carefully and the spectra taken are shown in Figure (24). Figure (24a) was taken with the dye laser tuned on the $45-4 p$ absorption. We see five distinct features here: four sharp lines and one broad one. The four sharp lines can be identified as atomic transitions. The fifth broad line can be identified as a molecular transition. Note that the fluorescence occurs at higher 


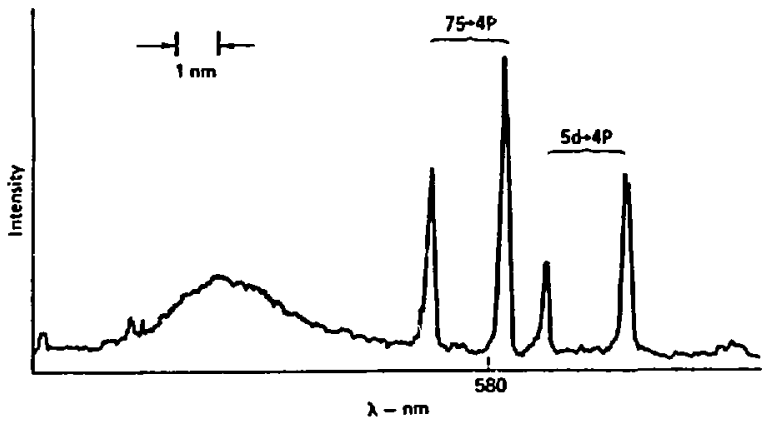

Figure 24a. The fluorescence from the $k$ vapor when pumped at $767 \mathrm{~nm}$, a) on the $4 \mathrm{~s}-4 \mathrm{p}$ transition, b) $.04 \mathrm{~nm}$ off of the $45-r p$ transition. 
energy than the pump. In Figure (24b) the dye laser has been tuned off of the atomic absorption line by approximately .04 nm. We see that all of the atonic features have disappeared and the molecular feature has increased in magnitude. Both scans were done under identical conditions and the vertical scales are the same.

These features can be explained if we look at the two sets of features, the atomic and the molecular, separately. Similar atomic features have been observed previously (45), (46) in sodium under quite similar conditions. In (46) it was argued that the producLion of high-lying states can result from energy-pooling collisions where formation of states higher than $2 h w$ requires conversion of kinetic energy. In our work the atomic features can be identifed as $5 d r 4 p$ and $7 s \rightarrow 4 p$ fluorescent transitions as shown in Figure (2). The energy of these upper states is $30185 \mathrm{~cm}^{-1}$ and $30274 \mathrm{~cm}^{-1}$ respectively which is greater (by more than $4000 \mathrm{~cm}^{-1}$ ) than twice the laser pump energy of $130<3 \mathrm{~cm}^{-1}$. This means that 4000 $\mathrm{cm}^{-1}$ (.5 ev) of energy must come from collisions. In (45) similar energies were required to achieve the obseryed Na transitions. One difference between our experimental conditions and those of both (45) and (46) is the significantly higher pressures used. We worked at an atomic density of about $10^{16}$ atoms $/ \mathrm{cm}^{3}$ while both (45) and (46) worked at pressures of about $10^{13}$ atoms/ $\mathrm{cm}^{3}$. We would, therefore, expect more collisions and also collisions of higher energy transfer due to the higher temperatures involved $\left(620^{\circ} \mathrm{K}\right.$ v.s. $500^{\circ} \mathrm{K}$.) We would, however, only 


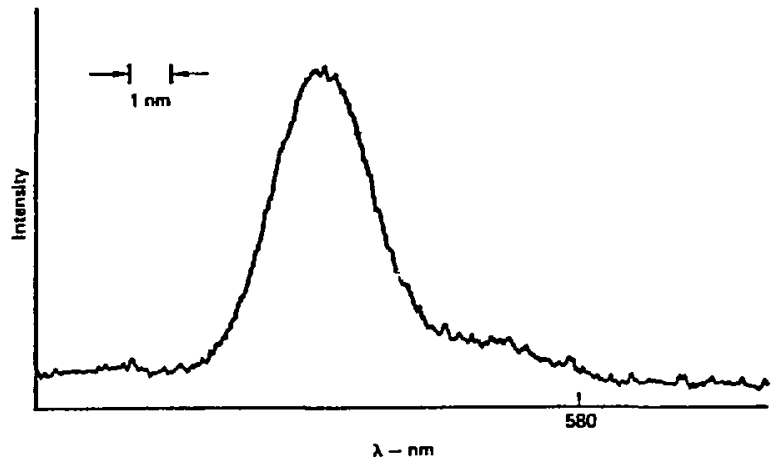

Figure 24b. The fluorescence from the $K$ vapor when pumped at $767 \mathrm{~nm}_{3}$ a) on the $4 \mathrm{~s}-4 \mathrm{p}$ transition, b) .04 $n m$ off of the $4 ;-4 p$ transition. 
expect $.025 \%$ of the atoms to have kinetic energy in excess of 4000 $\mathrm{cm}^{-1}$.

The molecular feature in Figure (24) appears structureless in the limit of our experimental resolution (2 A). This feature was observable both with the laser tuned onto the atom resonance line and with the laser tuned off by $.04 \mathrm{~nm}$. The intensity decreased as the pump laser was tuned on the line which could be the effect of less laser energy being available for the molecular species to absorb. This molecular feature was first observed by Rebbeck and Vaughn (47) in a low power discharge o',erating at about $10^{16}$ atoms $/ \mathrm{cm}^{3}$. The main b id in their work was centered at $572.5 \mathrm{~nm}$ and was found to be structureless even at high dispersion. They reported a linewidth (full width at half-maximum) of $3 \mathrm{~nm}$ and a symmetrical line shape. We can see from Figure (24) that this is a very good description of the feature we have observed. The proposed mechanism for this feature is a $\Sigma_{v}^{+}$upper state decaying to a ${ }^{3} \Sigma_{u}$ dissociative lower state which results in two ground state atoms. The upper $K_{2}$ level must be excited by the energy-pooling in the collision between two $K_{2}$ molecules excited to the ${ }^{1} \Sigma_{u}^{+}$state by the laser. A tentative diagram for this process is shown in Figure (25). This upper excited state could also be created by the collision of an excited molecule with an atum or through multiphoton absorption. No attempt was made here to determine which of these processes were dominant. 


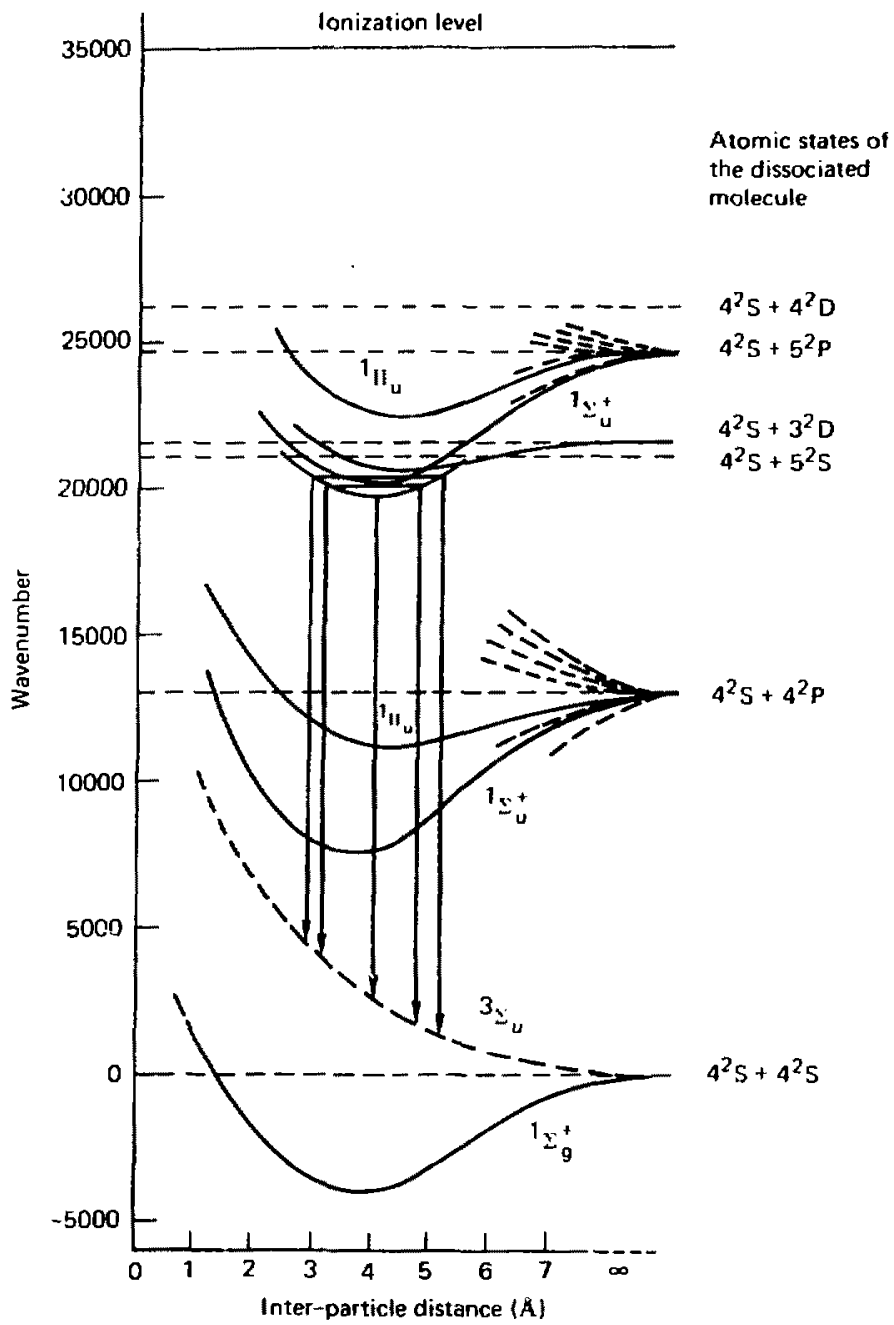

Figure 25. The diagram of the molecular patassium system showing the proposed dissociative ground state of the broad molecular feature. 
The molecular features are very interesting because if the lower state is a dissociating molecular state, we could pump the upper state more efficiently and, as in the eximer lasers, create a population inversion which could be used as a laser itself. More work, however, will need to be done on the excitation mechanism before one could estimate the potential efficiency of such a device.

Some preliminary studies were done on the effect of pressure and pump intensity on the atomic transitions, but were discontinued because of priorities of other work.

The next experimental results discussed are those from the brief experiments with the opto-galvanic effect. This effect has been observed experimentally as long ago as 1925 (48), but was first applied as we used it in Reference (49). The method used was outlined in the last chapter. We see in figure (15) the results of a scan of the dye laser which excited $4 p-7 s$ and $4 p-5 d$ transitions in the potassium vapor of the hollow-cathode tube. We clearly see the components of these transitions. This technique appears ideally suited for atomic absorption spectroscopy as described in (50) and to lock a c.w. dye laser (51). This last possibility holds great promise for locking a laser to the desired u.v. absorption line. This would create a very stable, perfectly tuned pump system for work involving atomic excitation. This technique should be easily extendable to use with pulsed laser system as well is the demonstrated c.w. systeris. This technique 
was intended to be used to tune our laser to the correct transition but was never used for this purpose.

The last visible spectroscopy results are crucial to the understanding of deexcitation dynamics in the potassium vapor. These spectra give us an idea of the path of cascading as the upper excited state is depopulated by stimulated emission. The experiment was set up as described in the last chapter. The first spectra were taken without the aid of the boxcar integrator. The output of the photomultiplier tube was put into a picoammeter and used the internal time constant of the picoammeter to average the pulses of the pump laser. The monochrometer was scanned over a $200 \mathrm{~nm}$ range from $400 \mathrm{~nm}$ to $600 \mathrm{~nm}$. The recorded ou iput is shown in Figure (26). We see several sharp features as well as a few broader lines and a yeneral offset of the whole spectra. We would expect to see many if not all of the visiole transitions in the potassium because as can be see from Figure (2), all of the transitions in the $400 \mathrm{~nm}$ to $600 \mathrm{~nm}$ region are the result of $\mathrm{ns}-4 \mathrm{p}$ or nd-4p transitions. Since the $13 p$ level is being populated, we should see a series of lines beginning at about $475 \mathrm{~mm}$ and continuing up to $584 \mathrm{~nm}$. These features can be seen in Figure (26), but the issue is quite confused due to other spectral features. For instance the broad featureless molecular line discussed earlier at $572.5 \mathrm{~nm}$ can also be seen. We also see a very broad band centered in the $440 \mathrm{~nm}$ region where there is a well known molecular potassium band (52). Since molecular features should have characteristic lifetimes much longer than atomic features that are 


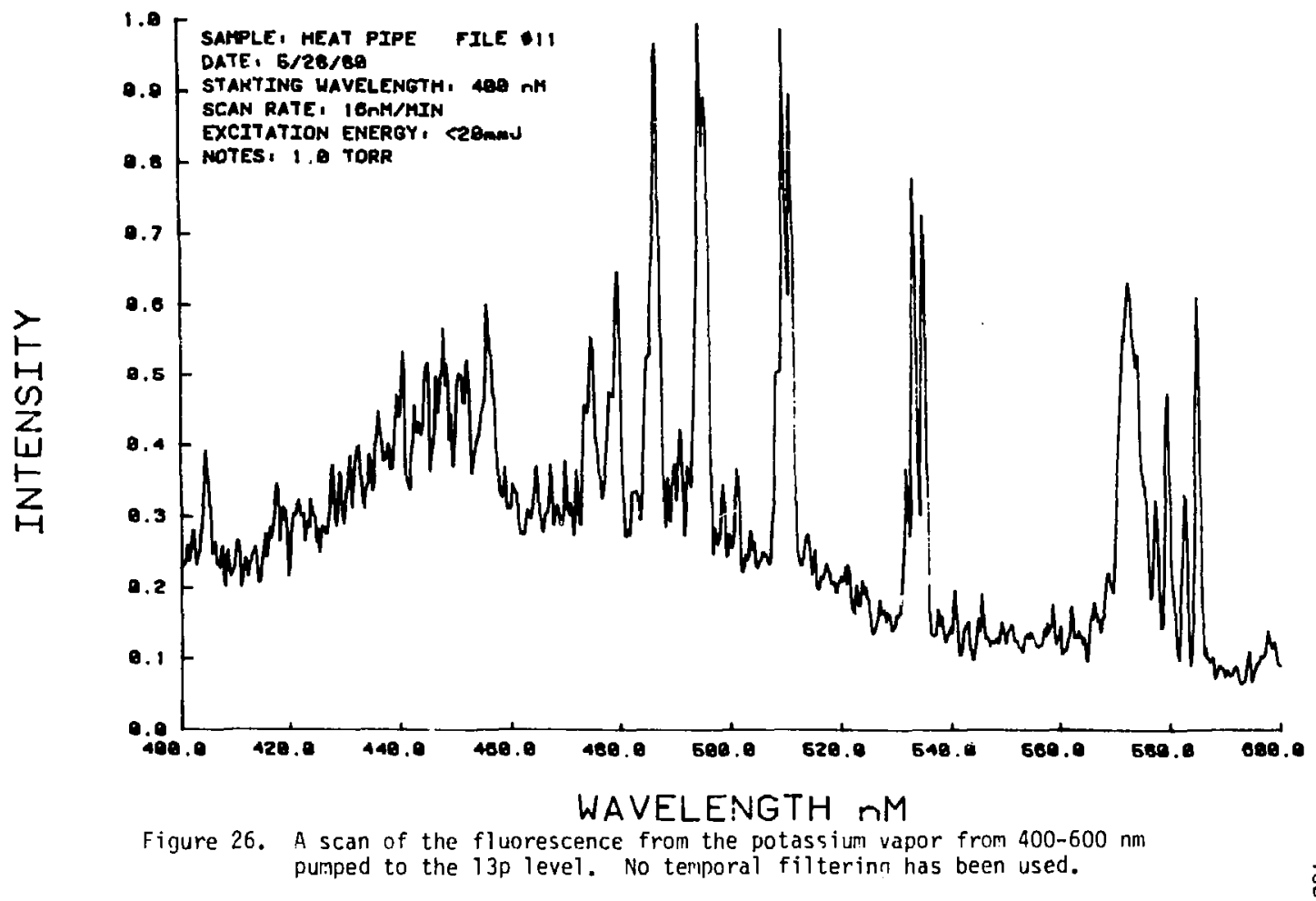


collisionally controlled, te expected to be able to filter out these features in the time domain by using the boxcar integrater with the sampling sate delayed very little from the laser exciting pulse. Figure (27a) shows a similar scan to figure (26), but now using the time discrimilation of the boxcar. A dramatic drop in the background interference and the almost total absence of the molecular features such as the 572.5 line is shown. Figure (27b) shows the same scan with the heaters on the heat pipe turned off. The large line at $585 \mathrm{~nm}$ is residual fundamental dye laser light getting through the Corning filter and provides an excellent wavelength marker for calibration of the spectra. In the sca:; Figure (27a), this line is the farthest to the long wavelength end of the scan. He begin to lose the $n s-4 p$ lines as we move to higher $n$ states. This is an indication that we have no population of these states and supports our calculations that the lld level will be populated in favor of the $13 \mathrm{~s}$ level in the initial superfluorescent transition out of the upper excited state. As we move to lower $n$ values the $n s-4 p$ and $n d-4 p$ transitions seem to becoms comparahle in magnitude, with the $7 \mathrm{~s}-4 \mathrm{p}$ actually larger than the $5 d-4 p$ transitions. We also see a hint of some transitions originatiag in a state higher than 13s. As we will see later, we have some excitation, presumably from collisions, to the $14 \mathrm{~s}$ state and would expect a comparable population of the 12d level. The separation between the $4 p 1 / 2$ and $4 p 3 / 2$ states naturally remains the same for all transitions, but since we are displaying the daca in wavelength and not energy, the splitting appears to decrease as 


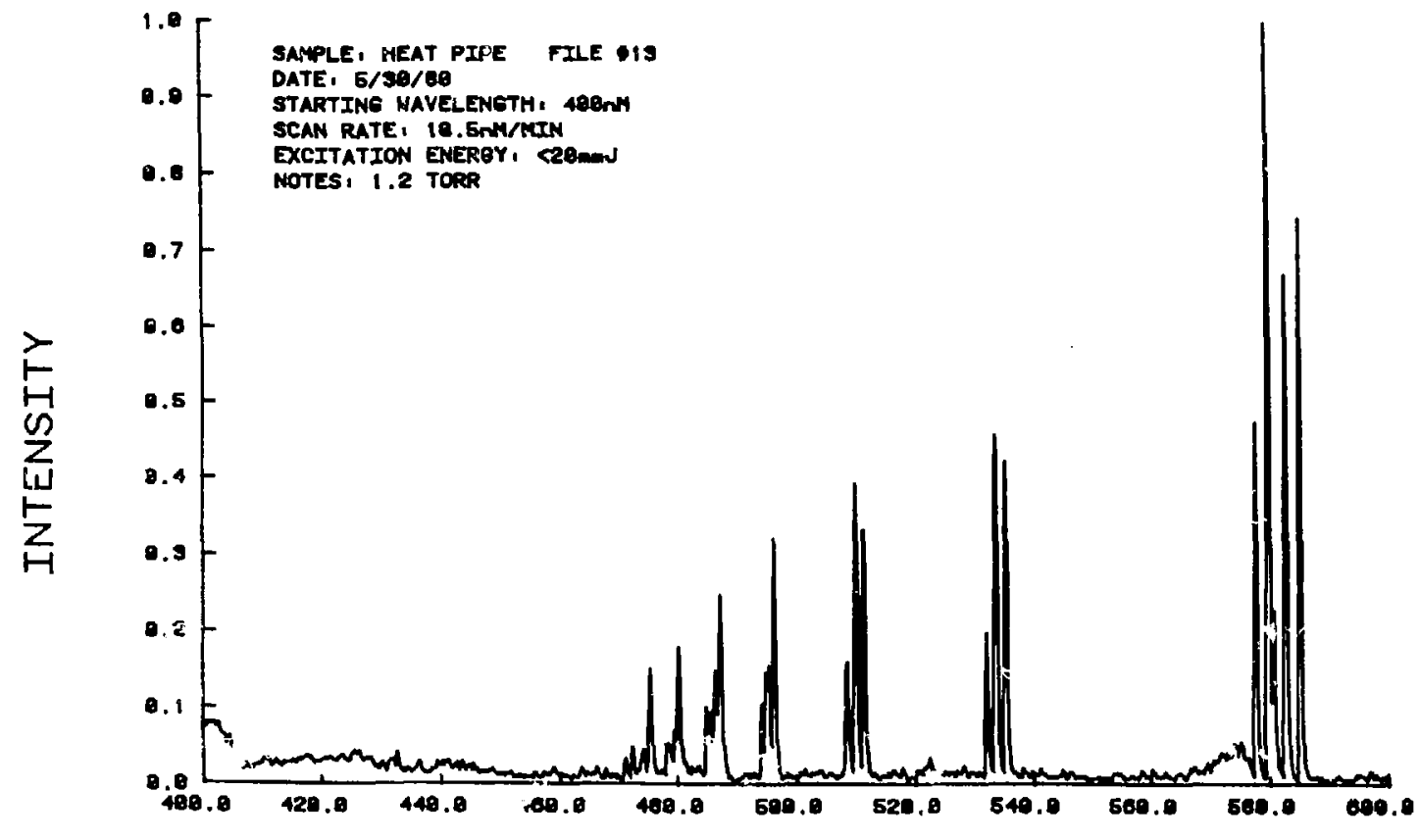

WAVELENGTH RM

Figure 27a. The same scan as Figure 26, but with the addition of temporal filtering using a boxcar integrater, a) hot $k$ vapor, b) cold (for backmround sianal). 


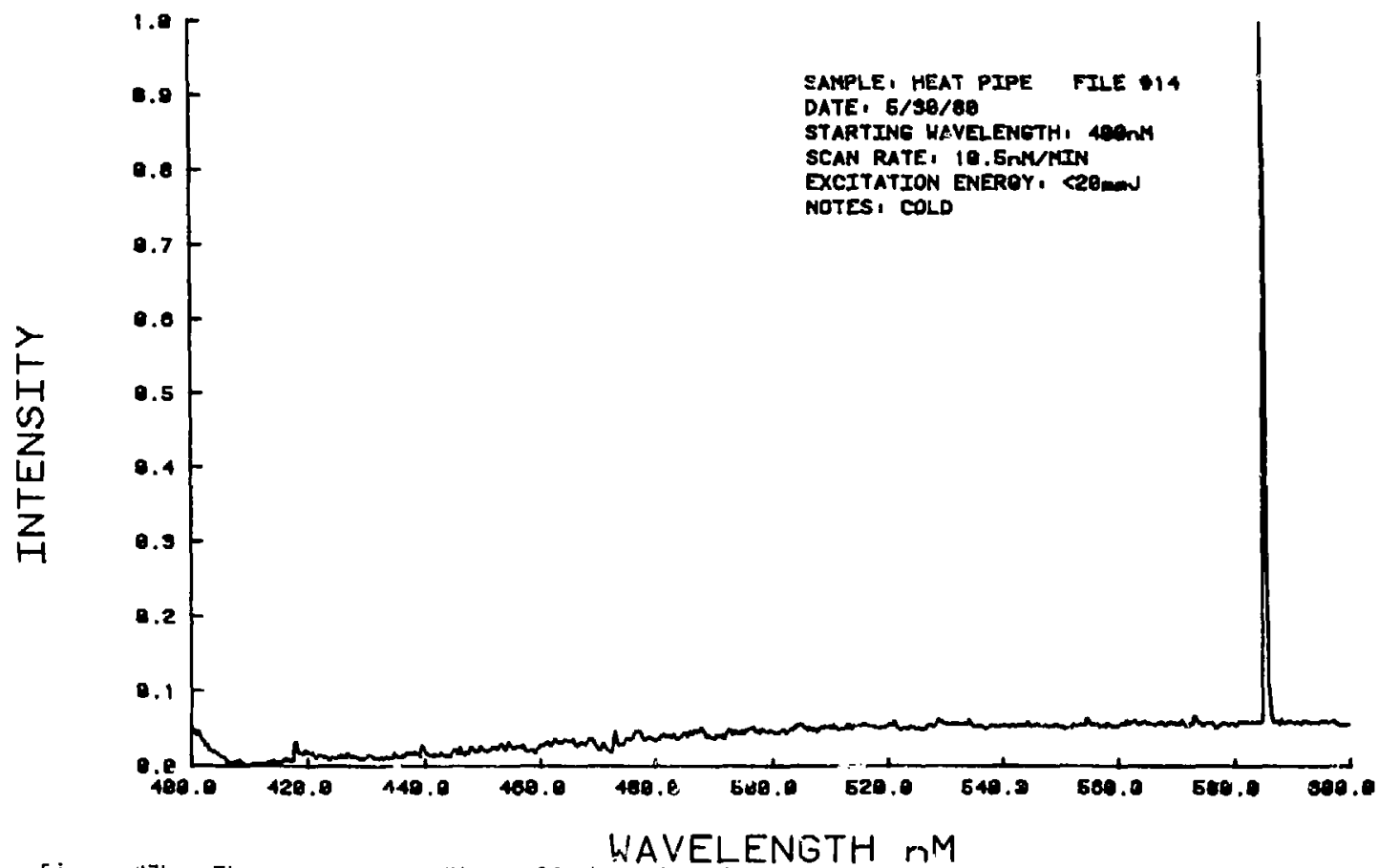

Figure 27h. The same scan as Figure 26, but with the addition of temporal filtering usind a boxcar integrater, a) hot $K$ vapor, b) cold (for backaround sinnal). 
we approach the high energy or short wavelength end of the spectrum. Figures (28a) and (28b) show a scan for $600 \mathrm{~nm}$ to $800 \mathrm{~nm}$. Once again the secc:. 1 of these scans was done with a cold heat pipe for reference. The large feature at $674 \mathrm{~nm}$ is the second order of the $337 \mathrm{~nm} \mathrm{~N} N_{2}$ laser off of the monocrometer grating. This is due to scatter in the dye laser cell. Once again this provides a convenient wavelength marker. We see here a large featureless line and the two sharp atomic lines due to the $6 s-4 p$ and $4 d-4 p$ potassium transitions. The extremely wide line is in both of the scans and is due to fluorescence of the Corning 7-54 filter. The next set of figures, Figures (29a) and (29b) show a complete scan at a resolution of $.5 \mathrm{~nm}$ from $400 \mathrm{~nm}$ to ${ }^{n} 00 \mathrm{~nm}$ with the addition of a filter to remove the $\mathrm{N}_{2}$ laser scatter. We can see clear?y the pattern of fluorescence from the excited states. One point should be noted. If Figure (29b) is carefully compared to Figure (29a) in the region of $695 \mathrm{~nm}$, we see that some of the fluorescent energy of the filter is absorbed by the atomic system or the molecular system associated with this atomic line. This indicates the very large cross section for these transitions.

It is now clear that virtually all levels below the pumped level (and possibly even the ones directly above it) are involved in the depopulation process. The information needed is the rate of the depopulation so we can predict the rate of depopulation of the upper lasing level. To achieve this we proceeded as was described in the last chapter. The output of the photomultiplier tube was viewed directly on the oscilloscope (Tektronix Mode? 


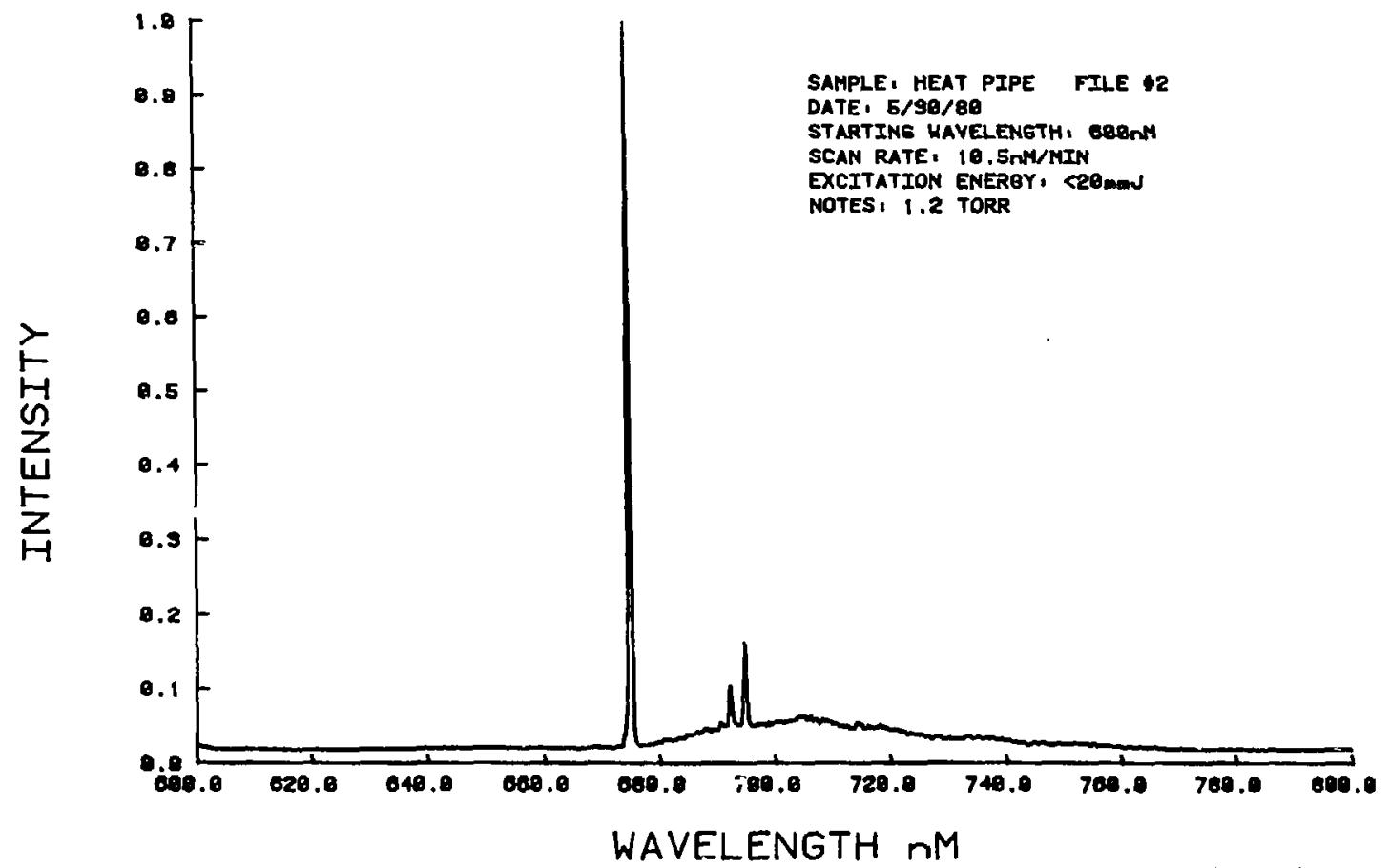

Figure 28a. The same as 27 , but from 600-800 nm. The 1 ine at $674 \mathrm{~nm}$ is the second grating order of scattered $337 \mathrm{~nm} \mathrm{~N}_{2}$ laser light, a) hot $\mathrm{K}$ vapor, b) cold. 


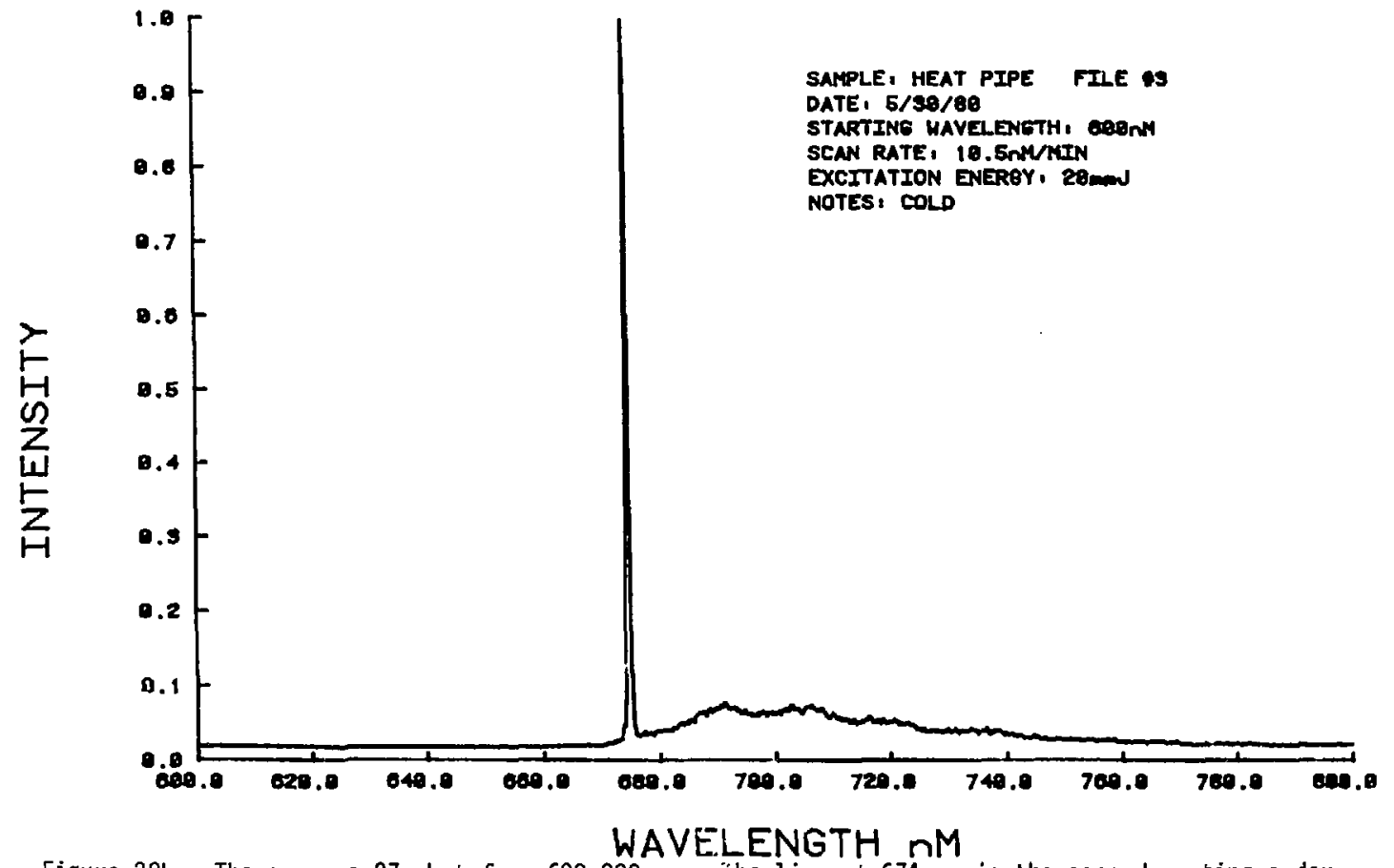

Figure 28b. The same as 27 , but from 600-800 nm. The line at $674 \mathrm{~nm}$ is the second arating order of scattered $337 \mathrm{~nm} \mathrm{~N}$ laser light, a) hot $\mathrm{K}$ vapor, b) cold. 


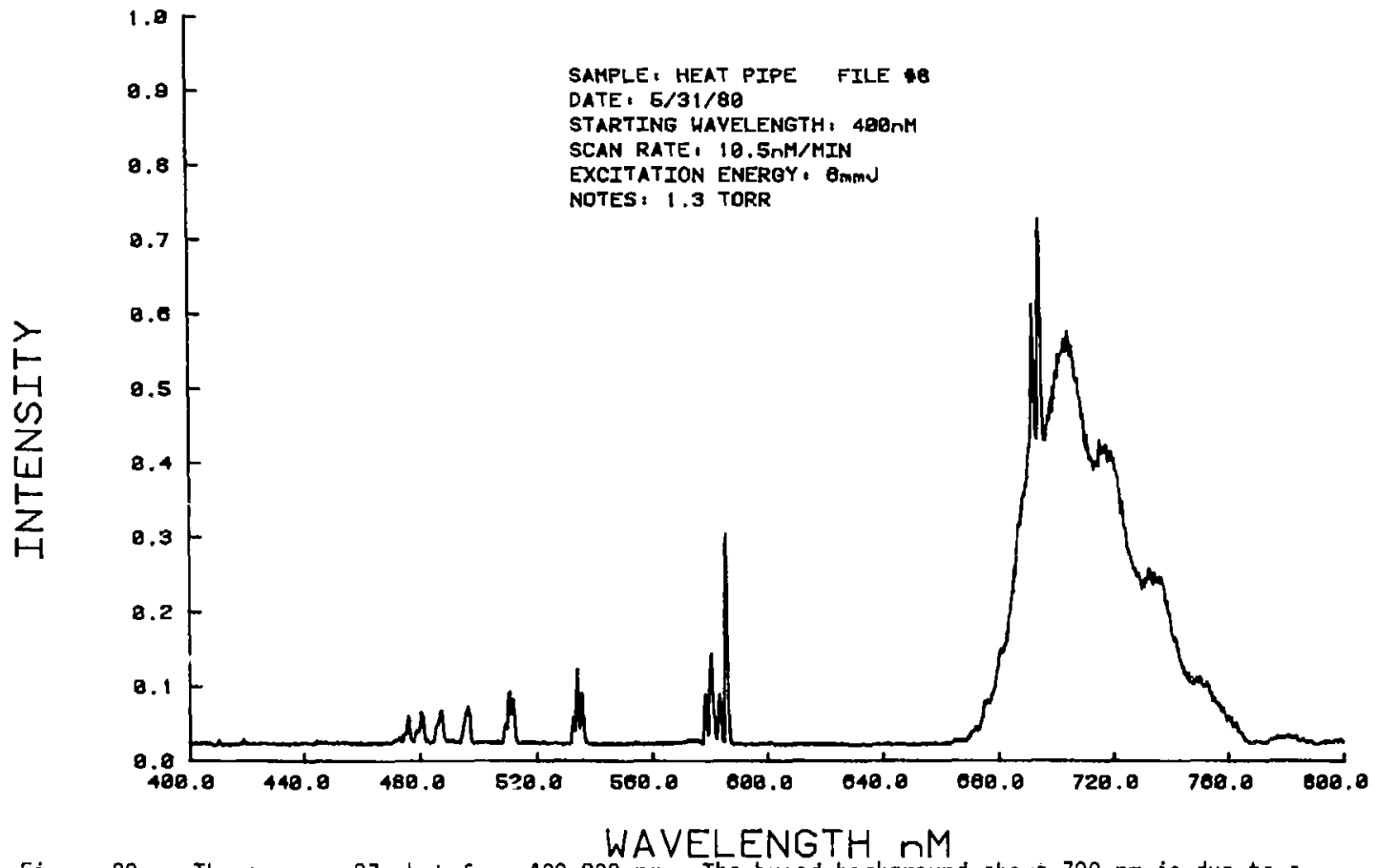

Figure 29a. The same as 27 , but from $400-300 \mathrm{~nm}$. The broad background about $700 \mathrm{~nm}$ is due to a fluorescing filter. Note the partial absorption of the broart fluorescence about the sharp peaks near $690 \mathrm{~nm}$, a) hot $K$ vapor, b) cold. 


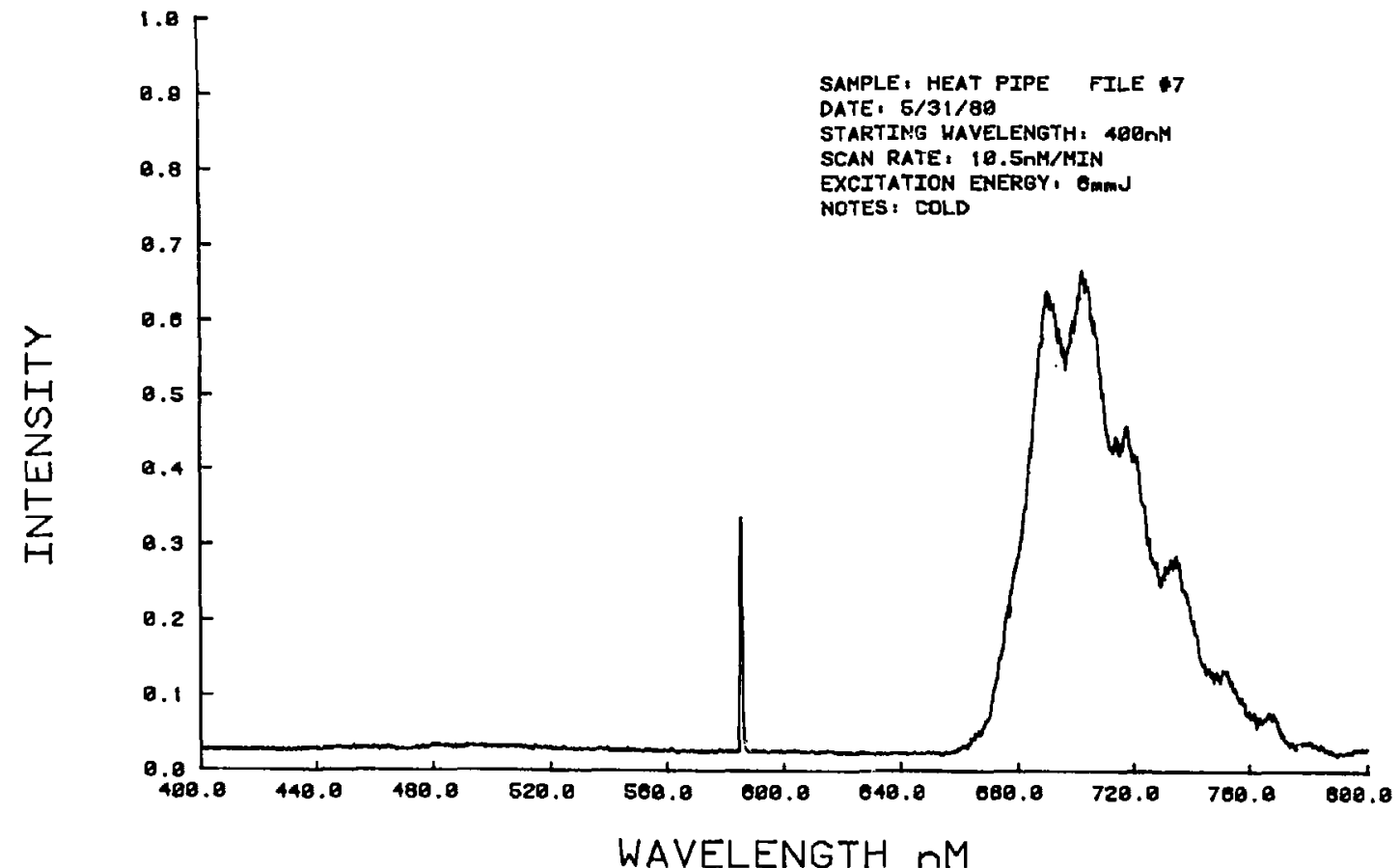

Figure 29h. The same as 27, but from 400-800 $\mathrm{nm}$. The broad background about $700 \mathrm{~nm}$ is due to a fluorescing filter. Note the partial absorption of the broad fluorescence about the sharp peaks rear $690 \mathrm{~nm}$, a) hot $K$ vapor, b) cold. 
;844) and pictures were taken of the trace. These pictures are shown in Figures $(30 a-30 j)$ for all of the wavelengths shown in Figure (27a). Because the monochror_ter slit widths had to be increased to let more light in to compensate for the lower response that is inherent in a fast measurement, the wavelength resolution was sacrificed and the individual transitions could not be identified in the $n s-4 p$ and $(n-2) d-4 p$ bunches. The wavelengths shown in Figures (30) are the monochrometer reading at the peak of the photomultiplier response. It is clear from figures $(30 \mathrm{~h})$ and (30j) which are pictures of the dye laser output, that the detection system has the capability to resolve the $10 \mathrm{nsec}$ pulses. In Figures (30a) through $(30 \mathrm{~g})$ we see that as we get to longer wavelengths or lower lying transitions, the pulse length becomes longer and the peak of the pulse is delayed longer. The depopulation rate in the higher lying transition indicates that they are being depopulatef by superfluorescent transitions. This observation agrees quite well with those in reference (53). This work was dore on the excited $s(n=12)$ and $d(n=10)$ states of rubidium with a system quite similar to ours. The main difference is the working densities of the vapor $\left(10^{12}\right.$ atoms $/ \mathrm{cm}^{3}$ vs. $10^{16}$ atoms $\left(\mathrm{cm}^{3}.\right)$ The results presented here are the first, time resolved, fluorescent results available for the excited states of potassium as far as is known.

The next main area of spectrascopic results for us to discuss are the results in the FIR region of the spectrum. By using the techniques discussed in the last chapter, we were able to deveiop 

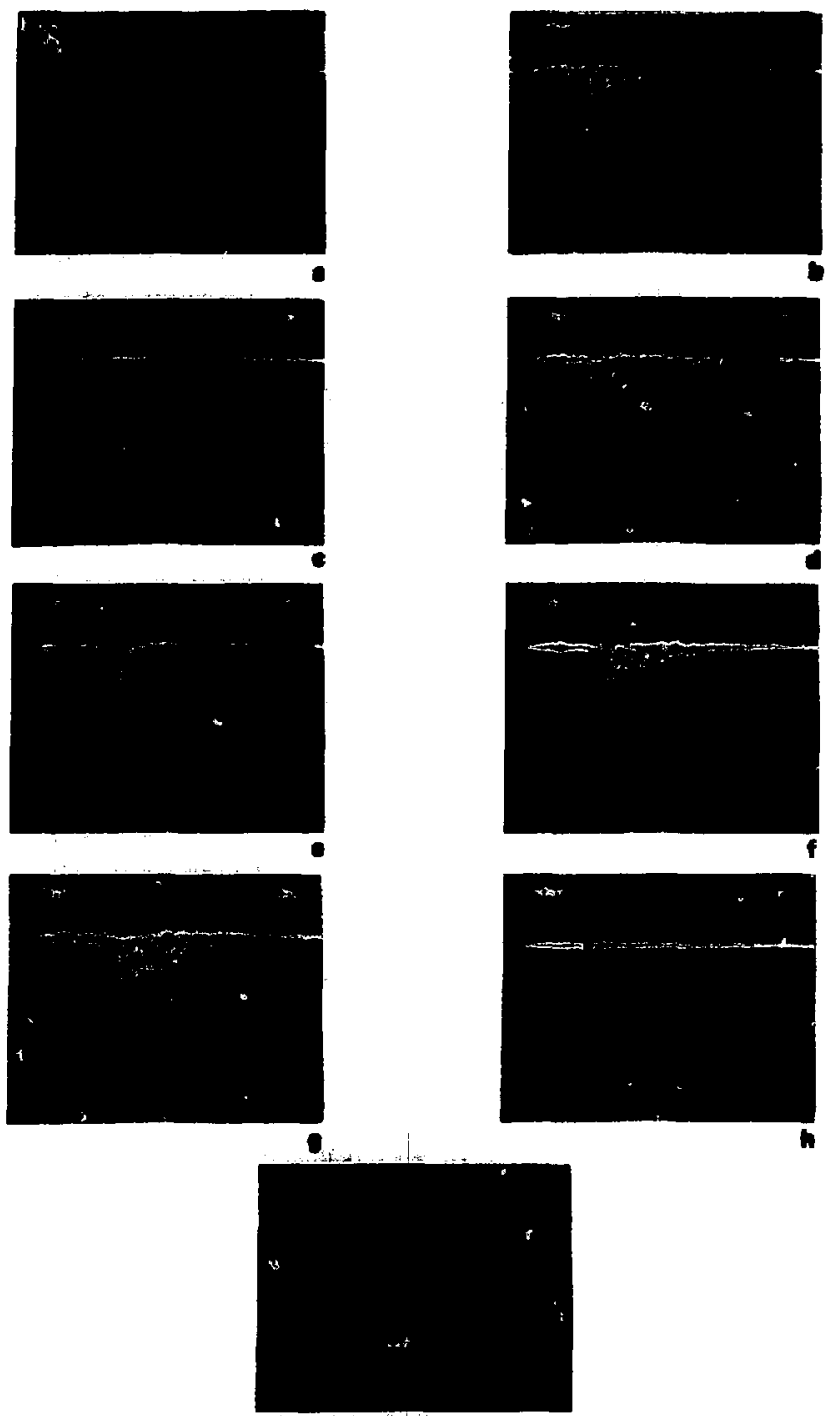

Figure 30. Photographs of the temporal character of the groups of lines shown in Figure $27 \mathrm{a}$, a) $475.4 \mathrm{~nm}$, b) $4802 \mathrm{~nm}$, c) $486.5 \mathrm{~nm}$ d) $495.3 \mathrm{~nm}$, e) $510.8 \mathrm{~nm}$, f) $533.2 \mathrm{~nm}$, g) 579.8 $\mathrm{nm}, \mathrm{h}) 585.5 \mathrm{~nm}$, i) same as (h), but on a $20 \mathrm{nsec} / \mathrm{cm}$ scale. 
a spectroscopic method which could resolve shifts or splittings in the FIR of less than $.3 \mathrm{~cm}^{-1}$ after several improvements. Here we will look at results using both of our FIR detectors and also at the FIR spectrum of water vapor.

As described in Chapter III, the output of the FIR detector was monitored by the lock-in amplifier whose output was sampled and digitized by a digital voltmeter and recorded by the computer. Figure (31) illustrates a typical interferometric output when the instrument was run at low resolution. We see here that we have a definite periodic signal which is being modulated by at least one other signal beating against it. If we had only one frequency, we would expect a pure sine wave whereas if we had a very broadband signal we would expect to see only one spike corresponding to the zero difference position of the two arms of the interferometer. In Figure (31) we see we have something between these two extremes, and therefore, expect several distinct features. We also see here that we have a large d.c. offset and a shifting of the average offset. These are due to less than perfect alignment and will show up as a large first Fourier component and low frequency noise respectively.

When the output of interferometer is processed by the computer to obtain the fast Fourier transform, we obtain a spectra such as that shown in Figure (32a). We see here several very sharp features as well as the predicted low frequency noise. The sharp features are more easily located in the expanded view of Figure (32b). These featl.es can be identified by reference to the 


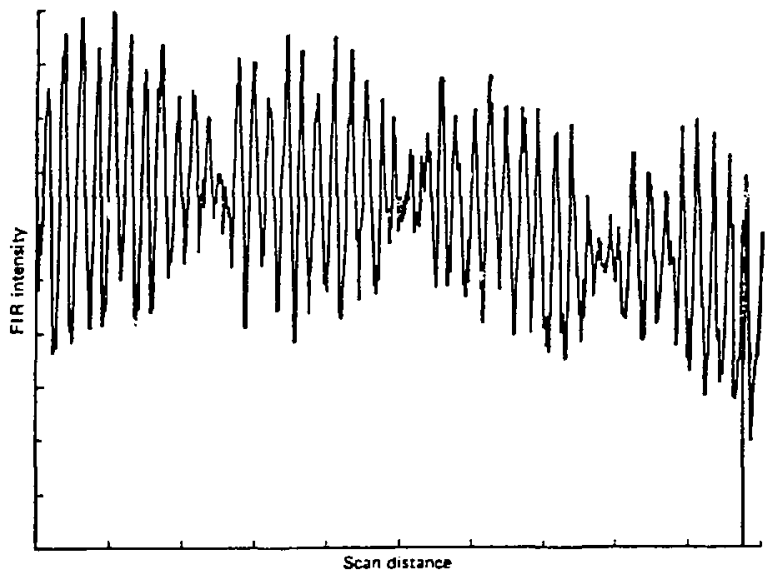

Figure 31. Typical output for a low resolution scan of the Michelson interferometer. 


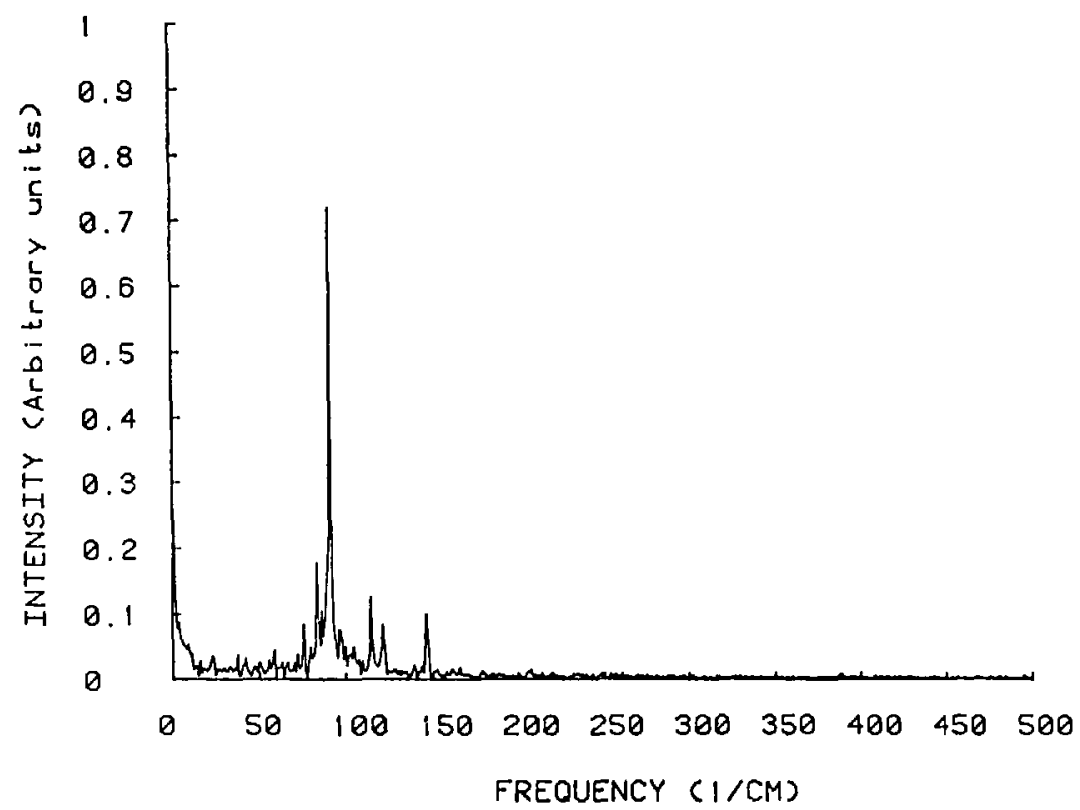

Figure 32a. Fourier transform spectrum of the output of the heat pipe using the Ga:Ge bolometer, a) total spectrum, b) expanded view of (a). 


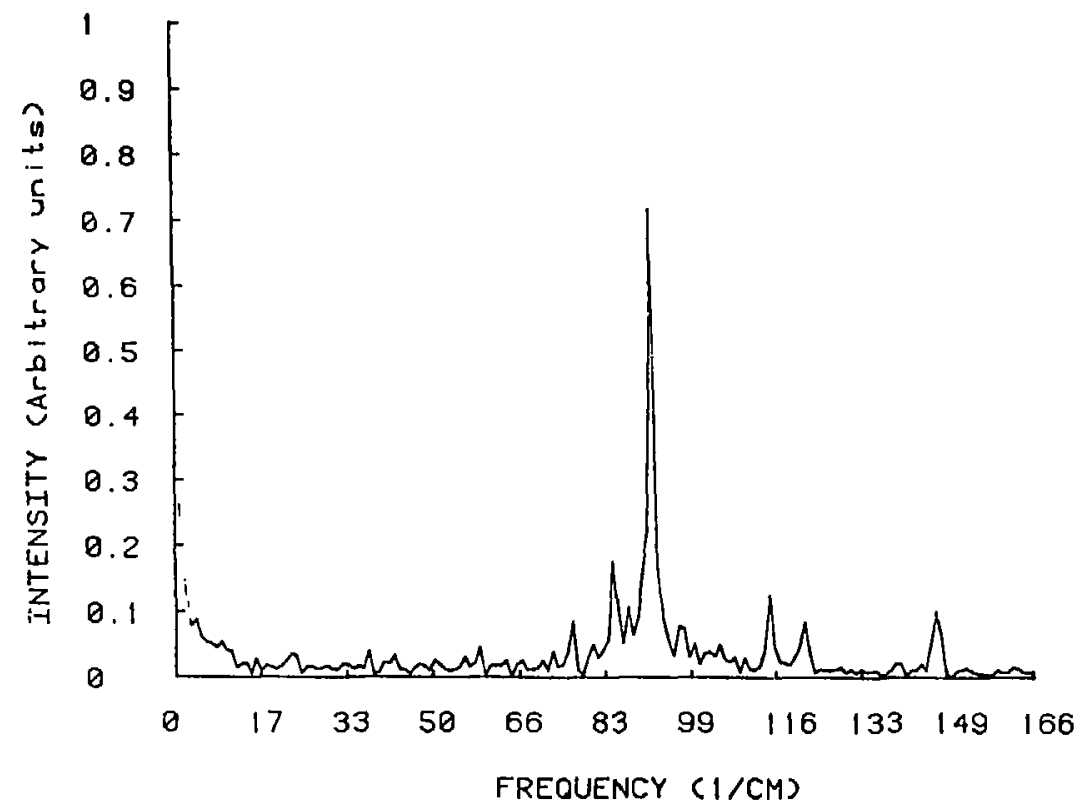

Figure 32b. Fourier transform spectrum of the output of the heat pipe using the $\mathrm{Ga}-\mathrm{Ge}$ bolometer, a) total spectrum, b) expanded view of (a). 
expanded diagram of the upper excited states of potassium shown in Figure (3). Several identifications can be made in this spectrum, but there is still a problem of unresolved peaks in the region about $90 \mathrm{~cm}^{-1}$. The resolution was increased by taking our clata points at longer time intervals which allowed us to improve the resolus.ion by a factor over three. This comes from the fact that we are scanning over more fringes and the resolution is directly proportional to that number. A scan at this higher resolution is shown in Figure (33). This spectra was taken with the Golay cell as the detector and the heat pipe pressure was 1.0 Torr. The time constant was 10 seconds and the total running time for the scan was 171 minutes. The peaks have been clearly resolved and the appropriate identifications $c$ an be made. The largest peak is measured to be $91.5 \mathrm{~cm}^{-1}$ and the calculated $13 \mathrm{p}-11 \mathrm{~d}$ transition energy is $91.6 \mathrm{~cm}^{-1}$. The two large peaks below the $91.5 \mathrm{~cm}^{-1}$ feature are measured to be $76.4 \mathrm{~cm}^{-1}$ and $84.2 \mathrm{~cm}^{-1}$. The 84.2 $\mathrm{cm}^{-1}$ peak is identified as the $11 \mathrm{~d}-12 \mathrm{p}$ transition predicted at $83.9 \mathrm{~cm}^{-1}$. The $76.4 \mathrm{~cm}^{-1}$ peak, however, can only be identified as the $14 \mathrm{~s}-13 \mathrm{p}$ transision calculated to be $76.1 \mathrm{~cm}^{-1}$. This apparent anomally in the spectrum can be understood when it is remembered that the thermal energy of the atoms is about 400 $\mathrm{cm}^{-1}$ so some collisional population of this state would be expected. The emission might appear as stimulated emission then, because the $13 p$ state very rapidly deexcites and we are left with a population inversion in the $14 \mathrm{~s}$ state. The curiosity is, therefore, not that we see emission from the $14 \mathrm{~s}$ state, but that we do 


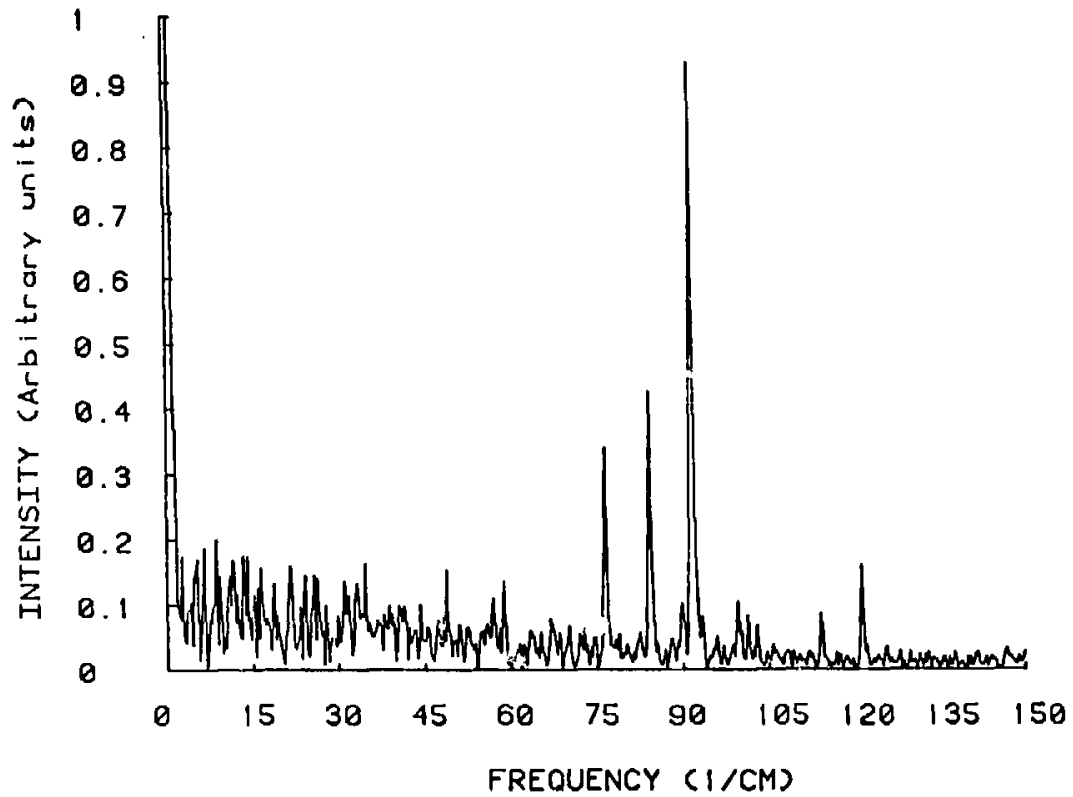

Figure 33. High resolution scan of the heat pipe output using the golly cell.

$\overrightarrow{5}$ 
not see any emission from the 12d state. Neither of the nearest neighbor transitions appear.

Two other features that can be identified are those at 114.1 $\mathrm{cm}^{-1}$ and $121.2 \mathrm{~cm}^{-1}$. These correspond exactly to the 114.1 $\mathrm{cm}^{-1}$ and $121.2 \mathrm{~cm}^{-1} 10 \mathrm{~d}-11 \mathrm{p}$ and $12 \mathrm{p}-10 \mathrm{~d}$ transitions respectively. There are two significant absences fram our spectri that should also be discussed. At no point do we see the cascading process include the upper excited ns states. We have the doility ere to see any transition populating the $11 \mathrm{~s}$ or higher states, but no significant signal at $79.2 \mathrm{~cm}^{-1}$ or $103.3 \mathrm{~cm}^{-1}$ is seen. The other absence noted here is the $146.7 \mathrm{~cm}^{-1} 11 \mathrm{~d}-10 \mathrm{f}$ peak observed in the lower resolution scan. This can easily be accounted for by the fact that the crystal quartz window on the Golay detector has a broad band absorption which apparently absorbs this output peak. Scans done with the bolometer such as Figure (34) show this peak quite distinctly. The scan in Figure (34) was done at .3 Torr in the Stark cell heat pipe oven described described in Chapter III. This is wiul the flat or "pancake" design. The dramatic improvement in signal to noise can be seen which is made more impressive when it is noted that the time constant for this scan was 3 seconds as opposed to the 10 second time constant of Figure (33). This allowed us to complete the scan in less than 52 mirutes, one-third of the time for the Golay cell. The relative peak heights of these two scans have changed because of the change in pressure. This indicates a strong collisional effect on the 11d-12p and 14s-13p transitions, but very little 


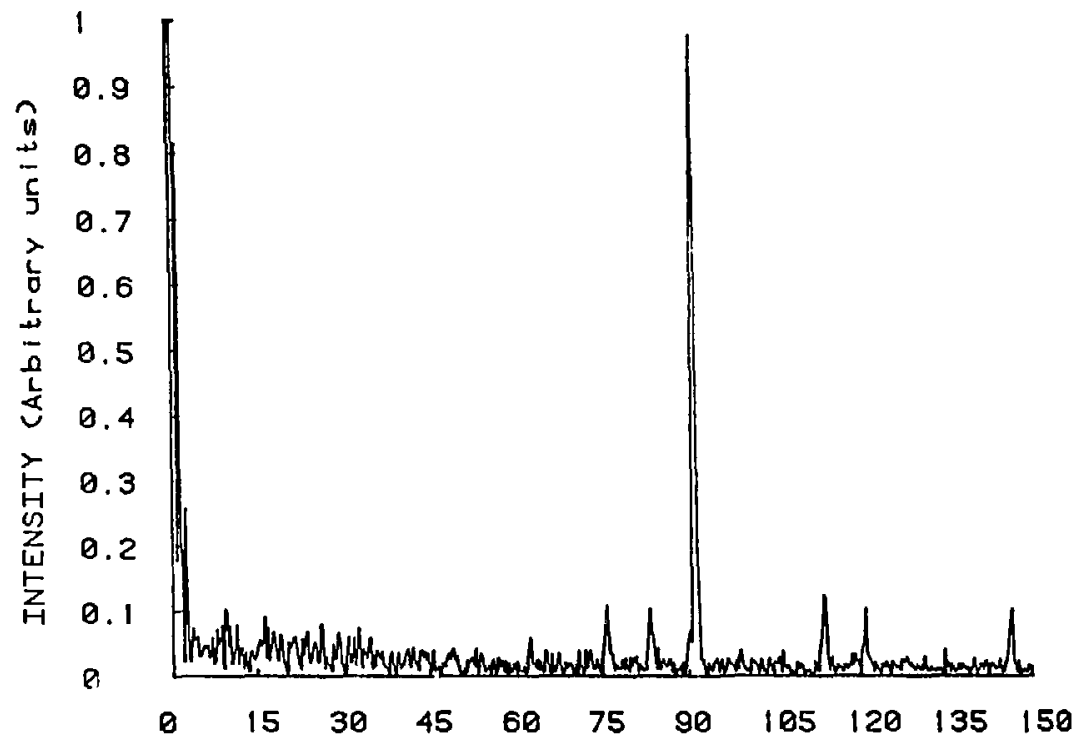

FREQUENCY (I/CM)

Figure 34. High resolution scan of the "pancake" heat pipe output with no field using the bolometer.

$\bar{N}$ 
effect on the cascading further down in the system. This goes dlong with what one would expect. The lower the level, the smaller its effective cross section, and therefore, the less effect pressure changes should have on its population.

Using the same conditions as those used in Figure (34) the pulsed electric field was applied. After tuning the dye laser to the transition once again, because the 13p level had shifted about $.53 \mathrm{~cm}^{-1}$, the interferometer was scanned. Three scans were taken alternately with scans of the heat pipe output with no field applied and the results of the scans with the fieid on were averaged as were those with the field off. The linewidths of the emission lines are seen to be similar, consisting of about four experimental points. The averaged results are shown in Figure (35a). A shift in the spectral lines can be seen quite clearly. The region from $88 \mathrm{~cm}^{-1}$ is shown on an expanded scale in Figure (35b) and illustrates both the excellent signal to noise of the measurements and the clear shift of $.3 \mathrm{~cm}^{-1} \pm .15$ to the higher frequency when the field is applied.

The last spectroscopic results discussed are the measurements done to characterize the response of the Ga:Ge bolometer. This was accomplished as described in Chapter III. In Figure (19), we see the spectra taken with the hot wire source and in Figure (36) this is shown expanded. This scan has a resolution of $1 \mathrm{~cm}^{-1}$. Because the work was done at atmospheric pressure, the water absorption lines which are very broad, and therefore, overlap over much of the spectral range covered. It is seen from this figure 


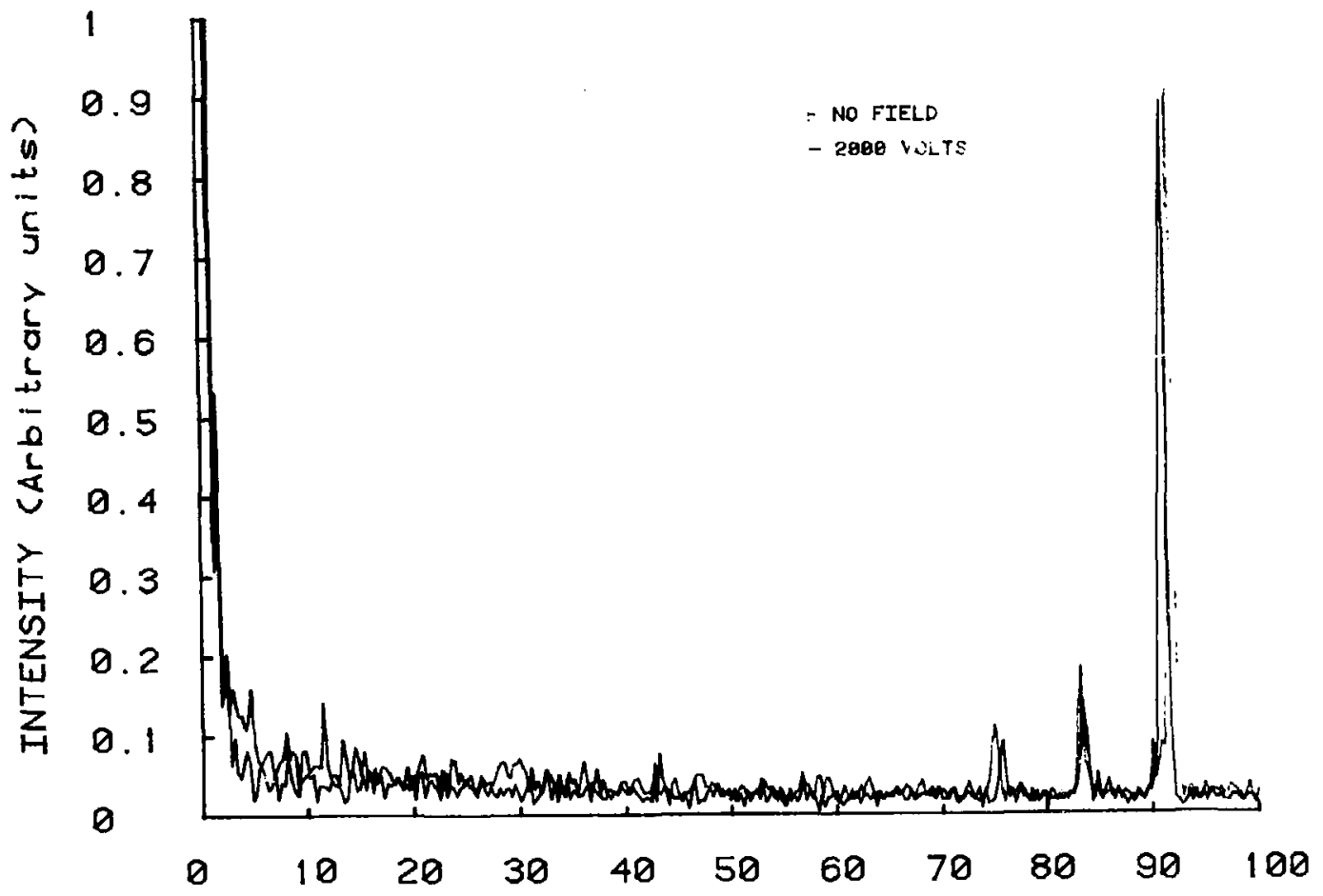

FREQUENCY ( $1 / \mathrm{CM})$

Figure 35a. Output of the "pancake" heat pipe with the field on $(\sim 2000 \mathrm{~V} / \mathrm{cm})$ and off, a!

$\vec{N}$ from $0-10 \mathrm{~cm}^{-1}$, b) from $83-120 \mathrm{~cm}^{-1}$. 


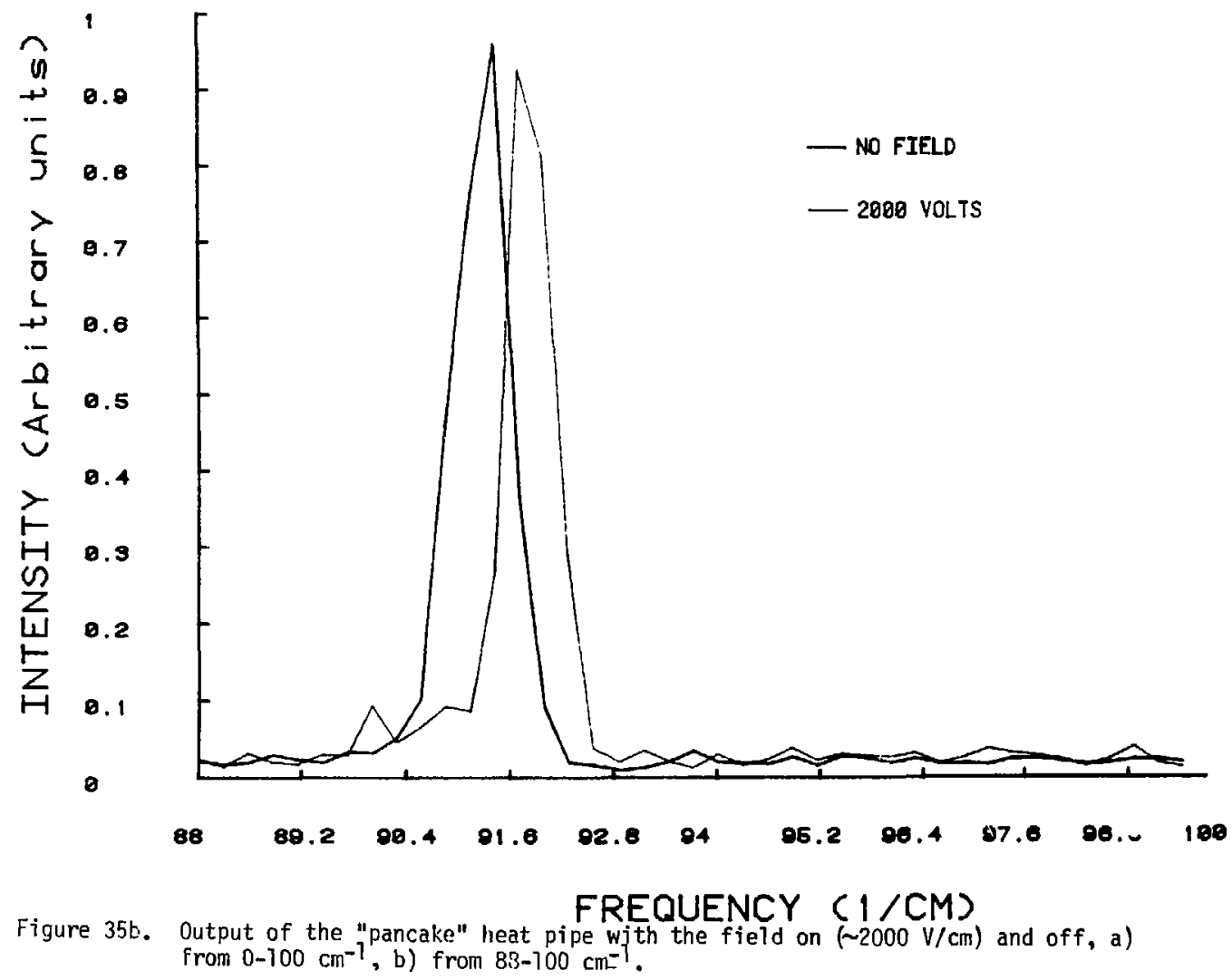




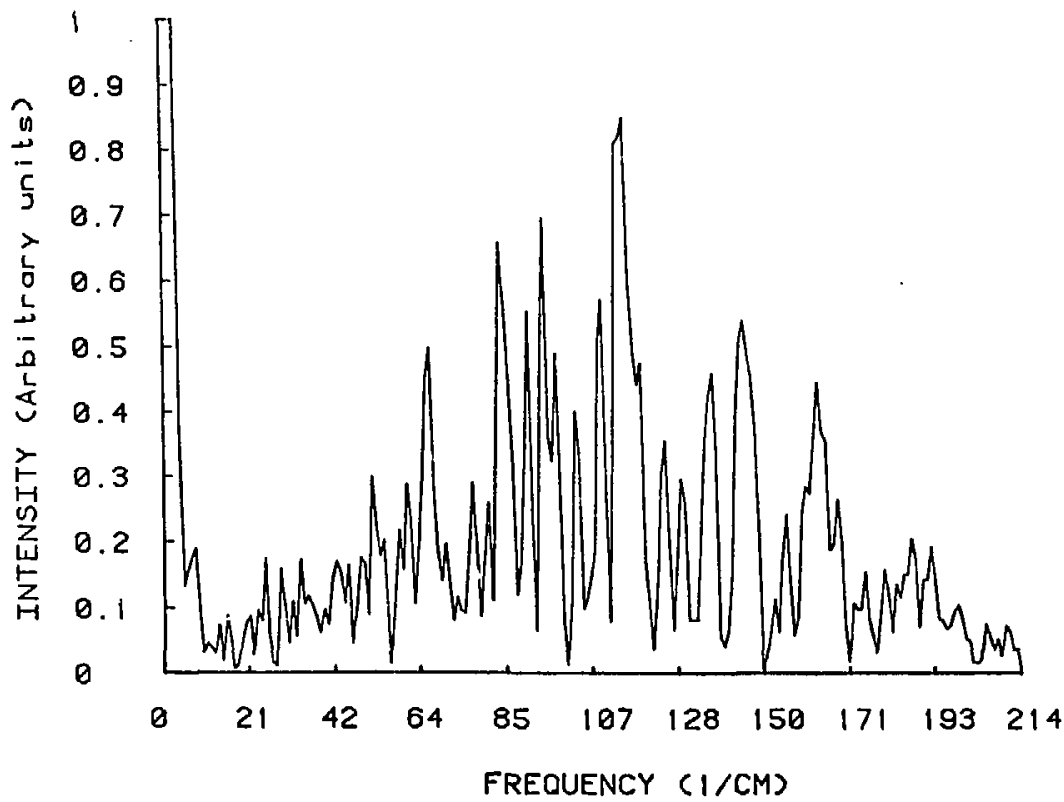

Figure 36. Expanded view of Figure 19 showing more clearly the water vapor absorptions.

wm 
that the detector, whose response is smooth in this region, has its peak response in just the right region for our purposes and does rot significantly distort the relative peak heights of the important lines whose energy lies between $75 \mathrm{~cm}^{-1}$ and $150 \mathrm{~cm}^{-1}$. 
V. DISCUSSION AND CONCLUSIONS

In this chapter the conclusions based upon the experimental results wil? be presented, problems concerning the experiment will be discussed, and recommendations for future work will be made.

By comparing the results of our experiments to the theory, an insight into the validity of the approximation methods used in the theory is obtained and the potential shortcomings of the experimental procedure are shown. The results of these comparisons will then point the way for improvements in our understanding of the problem.

We begin by comparing the results of the FIR spectroscopy to the predicted levels and shifts. The dominant transition in the experimental work is the 13p-11d transition which accounts for as much as $2 / 3$ of total FIR power out of the system as measured by the Michelson interferometer. As shown in Chapters II and IV the magnitude and direction of the shift agree well within the experimental limits outlined. This is a major result because it shows that substantial amounts of FIR radiation which is tunable over a potentially wide region of the spectrum can be generated by using several transitions and more than one atomic system. This can help extend the laboratory measurements of the rotational levels of molecules which would then help with the development of this branch of physics. Diatomics such as $\mathrm{HCl}$ and $\mathrm{CO}$ have spectral lines throughout this part of the spectrum.

We have, however, observed one level shift which does not agree with the predictions of Chupter II. In particular, we see 
that the predicted shift for the $11 \mathrm{~d}-12 \mathrm{p}$ transition is $-.66 \mathrm{~cm}^{-1}$ yet our measurement shows a shift of $-.24 \pm .15 \mathrm{~cm}^{-1}$. This discrepancy cannot be accounted for in the theory. The theoretical results for this shift seem reasonable. We would, therefore, hold the experimental results suspect. This, however, is not satisfying either because of the good agreement at other wavelengths and because of the extreme care used to insure the accuracy of the measurements. We, therefore, have a result which we are not comfortable with and which needs more experimental verification.

The unshifted FIR transition energies are well within the experimenta? errors of those energies predicted by the Rydberg formula. As mentioned earlier these predicted values will be much better as the value of $n+1$ gets larger.

The next areas to look at are the results of the visible spectroscopy. It was seen that as we come down in $n$ value of the upper fluorescent state, the time constant associated with that level increases. This also is quite consistent with the physical picture of the system we have described. The stimulated processes become decreasingly important as we move to lower transition energies and the system becomes much less organized in terms of the excited state location of the outer electron. The interesting, but not surprising absence of the resonant transition at 767 $\mathrm{nm}$ is explained quite simply as self-absorption by the potassium vapor. The decrease in energy emitted as we move to higher emission frequencies is explained by looking at the relative 
Einstein B coefficients for the upper level transitions. Once again because of their higher frequency, the transitions to low lying states are less likely, and therefore, the energy of the upper states goes mainly into nearby states. It was also seen how upper states can attain a significant population by energy transfer processes such as energy-pooling collisions. These processes are not well urderstood and have had very little experimental attention. The similar molecular process that has been observed is also not well understood. Both of these areas show great promise for helping unravel the physics of the collisions in excited states of atoms and molecules.

The last spectroscopic area is that of the opto-galvanic effect. This method of spectroscopy, while not explored extensively here, shows great promise as a tool in experimental work. Its very complex physical nature would seem to be a basic problem with its use as a theoretically assailable system. We have realized very large signal to noise ratios with very good reproducability from a simple system. These advantages have made this method of spectroscopy quite attractive and will continue to make it a useful adjunct to standard absorption spectroscopy for the location of transitions in many atomic systems.

The problems we have run into have been due to three main sources. The first is the difficulty in generating large amounts of narrow linewidth ultra-violet radiation in the $292 \mathrm{~nm}$ region of the spectrum. This has been recently overcome by the introduction of several commercial laser systems. These are the Nd:Yag pumped 
dye laser sysiems which are capable of delivering $10^{3}$ times the energy of our system in pulses of about the same temporal profile. Some of these systems offer excellent linewidth matches to our absorption linewidth and would prove nearly ideal for this application. Because of the linewidth match which could couple three times the energy in and the greater energy available, we would expect $3 \times 10^{3}$ greater output energy if there are enough atoms available in the vapor. This would require a longer device or higher pressure.

The second problem area has been the detectors used in the FIR. The detectors used in this work were long time constant thermal detectors which are inherently unsuited to the short pulse system we have developed. Currently there seem to be two alternative methods of detection which would be suitable for our use. These are a Shottky diode detector using a FIR laser as a local oscillator. An atomic beam of potassium pumped to the energy level desired and then ionized if radiation is absorbed. The latter method has been used extensively in the microwave and FIR regions and provides very low level detection with narrow linewidth resolution (54-56). A similar method has been used in (57) to detect infrared radiation by observing the visible output of the excited atomic system. These systems are very promising, but all have the disadvantages of size and complexity. No simple solution to the detection of the FIR at low levels seems to be at hand. There is one possibility for detection of the FIR which we have not yet mentioned. This is the use of pyroelectric 
detectors. These devices are capable of subnanosecond response times, but suffer from very low responsivities. This, however, would be somewhat alleviated with the higher signal levels available from a Nd: Yag system.

The last problem source has been the heat pipe oven used to Stark shift the atomic levels. The design which proved successfui had basic problems in the response of the structure to the thermal stresses applied at high temperature. A more complicated design based on the original Stark cell heat pipe appears to have the best chance of overcoming the protlems. However, the design used, if built in a sturdier fashion could be modifiad to give the experimenter an excellent test bed for the study of metals at high temperatures under widely varying conditions with excellent optical access to the experimental region.

The areas of further study then, are the atomic and molecular energy pooling processes and the experimental apparatus development necessary to achieve high resolution, time resolved FIR spectra. These areas hold great promise for the experimentalist interested in atomic and molecular processes.

We have seen that the theory of highly excited atoms, while not complete, gives very good results in its predictions for the position and shifts of the energy levels. The improvements made are chiefly due to the availability of fast digital computers to do the calculations such as the numerical integration of the radial wave function. As the theoretical calculation techniques nave improved, so have the experimental procedures and apparatus. 
In particular, the development of the tunable laser has made possible the study of atoms in a single highly excited state. We have shown that we can in turn make ather regions of the spectra available for study. The FIR source developed here will hopefully help bridge the gap in tunable sources that has previously existed. 


\section{REFERERICES}

1. D. C. Laine, Nature 191, 795 (1961).

2. J. Gelbwachs, R. H. Pantell, H. E. Puthoff, and J. M. Yarborough, App 1. Phys. Lett., 14, 258 (1969).

3. J. M. Yarborough, S. S. Susiman, H. E. Puthoff, R. H. Panteli, and B. C. Johnson, Appl. Phys. Lett., 15, 102 (1969).

4. B. C. Johnson, H. E. Puthoff, J. SooHoo, and S. S. Sussman, Appl. Phys. Lett., 18, 181 (1971).

5. M. A. Piestrup, R. H. Fleming, and R. H. Pantell, Ap2l. Phys. Lett., 26, 418 (1975).

6. K. H. Yang, J. R. Mnrris, P. L. Richards, and Y. R. Shen, App I. Phys. Lett., 23, 669 (1973).

7. Y. R. Shen, Appl. Phys. Lett., 23, 516 (1973).

8. T. 1. Brown and P. A. Wolff, Phys, Rev. Lett., 29, 362 (1972).

9. R. L. Aggarwol, B. Lax, H. R. Fetterman, P. E. Tannurwald and B. J. Clifton, J. Appl. Phys. 45,3972 (1924).

10. K. P. Koo anci P. C. Claspy, Appl. Opt., 18, 1314 1979).

11. D. H. Martin and K. Mizuno, Adv. in Phys. 25, 211, 1976.

12. C. Fabre and S. Haroche, Opt. Comm., 15, 254 (1975).

13. M. E. Movesyan, N. N. Badalyan, and V. A. Irodyan, ZLETF Pis'ma $\underline{6}$, 631 (1967) (Russian).

14. P. P. Sorokin and J. R. Lankard, Jour. Chem. Phys., 54, 2184 (1971).

15. D. R. Griscnkowsky, J. R. Lankard, and P. P. Sornt in, IEEE J. Quan. Elect., QE-13, 391 (1977).

16. A.M.F. Lau, W. K. Bischel, C. K. Rhodes, and R. M. Hill, Appl. Phys. Lett. 29, 245 (1976).

17. J. J. Wyne and P. P. Sorokin, J. Phys. B. 8,137 (1975).

18. C. J. Latimer, Contemp. Phys. 20, 631 (1979).

19. M. Gross, P. Gray, C. Fabre, S. Haroche, and I :1. Raimond, Phy.. Rev. Lett., 43, 343 (1979).

2C. O. R. Bates and A. Damgoard Phil. Trans. R. Soc. : ndon 242, 101 (1949). 
21. E. M. Anderson and V. A. Zilitis Optics and Spectroscopy (USSR) 16. 99-101 (1964).

22. M. 1. Petroshen and I. V. Aborenkov, Vestn. Lenigr. Univ. No. 5 141 (1954).

23. D. R. Hartree and W. Hartree, Proc. Roy. Soc. (London) A 166, 450 (19:3).

24. A. N. Nesmeyanov, Vapor Pressure of the Elements, N.Y. Academic 1963.

2E. C. E. Theodosiov, Minima in the emissian oscillator strengths of alkali Rydberg states, J. Phys. B. 13 (1930) L1-L5.

26. A. E. Siegman, An Introduction to Lasers and Masers, McGraw-Hill, 1971 .

27. M. Sargent, Laser Physics, Reading, Mass., Addison Wesley, 1974.

28. M. Zimnerman, M. G. Littman, M. M. Kash, and D. Kleppner, Phys. Rev., 20. (1979).

29. Myron Zimmerman, private communication

30. J. J. Wynne and P. P. Sorokin, J. Phys. B. $\underline{8}$ L37-L4L (1975).

31. A. M. F. Lau, W. K. Bischel, C. K. Rhodes, and R. M. Hill, Ap. Phys. Lett. 29 p 245 (1976).

32. C. R. Vidal and T. Cooper, J. Appl. Phys. 40 p. 3370 (1969).

33. G. M. Grover, T. P. Cotter, and G. F. Erickson, J. Appl. Phys. 35, $1990(1964)$.

34. C. R. Vidal and F. B. Haller, Rev. of Sci. Ins. 42, 1779 (1971).

35. R. W. Boyd, J. G. Dodd, J. Krasinsky, and C. R. Stroud, Ja, Optics Letters, $\underline{5}, 117(1980)$.

36. L. L. Steinmetz, J. H. Richardson, B. W. Wallin, and W. A. Bookless, presented at a Topical meeting on Picosecond Phenomena, May 24-26, 1978, Hilton Head, S.C..

37. J. H. Richardson, L. L. Sieinmetz, S. B. Deutscher, W. A. Bookless, and W. L. Schmelzinger, Z. Naturforsch $\underline{33 a} 1592$ (1978).

38. J. H. Richardson, L. L. Steinmetz, S. B. Deutscher, W. A. Bookless, W. L. Schmelzinger, Anal. Biochem. 97, 17 (1979).

39. L. L. Steinmetz, W. A. Bookless, and J. H. Richardson, Apl. Optics, to be published (1980). 
40. R. B. Green, R. A. Keller, G. G. Luther, P. K. Schenck, and J. C. Travis, Apl. Phys. Lett., 29, 727 (1976).

41. R. Bystroff, LLNL, Gen. Chem. Div., Private Communication.

42. D. H. Martin, Spectroscopic Tochniques, N. Y., Wi ley, 1967.

43. Tektronics 4050 Series R08 Signal Processing ROM Pack. 2 Instructions.

44. L. L. Steinmetz, Private Communications

45. M. Allegrimi, G. Alzetta, A. Kopystoynska, L. Moi, and G. Orriols, Opt. Comm., 22, 329 (1977).

46. G. H. Bearman and J. J. Leventhal, Phys. Rev. Lett., 41, 1227 (1978).

47. M. M. Rebbeck and J. M. Vaughan, J. Phys. B, $\underline{4}, 258$ (1971).

48. Paul D. Foote and F. L. Mohler, Phys. Rev. 26, 195 (1925).

49. R. B. Ereen, R. A. Keller, G. G. Lather, P. K. Schenck, and J. C. Travis, Appl. Phys. Lett., 29,727 (1976).

50. W. B. Bridges, J. Opt. Soc. Am., 68, 352 (1978).

51. R. B. Green, R. A. Keller, G. G. Luther, P. K. Shenck, and J. C. Travis, IEEE J. Quant. Elec., QE-13, 63 (1977).

52. S. P. Sinha, Proc. Phys. Soc. Ion., 5436 (1947).

53. F. Gounard, M. Hugan, P. R. Fournier, and J. Berlande, J. Phys. B., 12, 547 (1979).

54. T. F. Gallagher and W. E. Cooke, Appl. Phys. Lett., 34, 369 (1979).

55. T. W. Ducas, W. P. Spencer, A. G. Vaidyanathan, W. H. Hamiltan and D. Kleppner, App 1. Phys. Lett., 35, 382 (1979).

56. M. Gross, P. Gay, C. Fabre, S. Haroche, and J. M. Rainard, Phys. Rev. Lett. 43,343 (1979).

57. J. A. Gelbwachs, C. F. Klein, and J. E. Wessel, IEEE Jour. Quant. Elec. QE-16, $137(1980)$.

58. L. Hrubesh, LLNL, Private Communication, 1980.

59. Atomic Energy-Level \& Grotrian Diagrams Vol. 2, by Stanley Bashkin and John 0. Stoner, Jr., North-Holland Pub. Co., Ams terdam, 1979. 


$$
\text { A P P NDICES }
$$




\section{APPENDIX A}

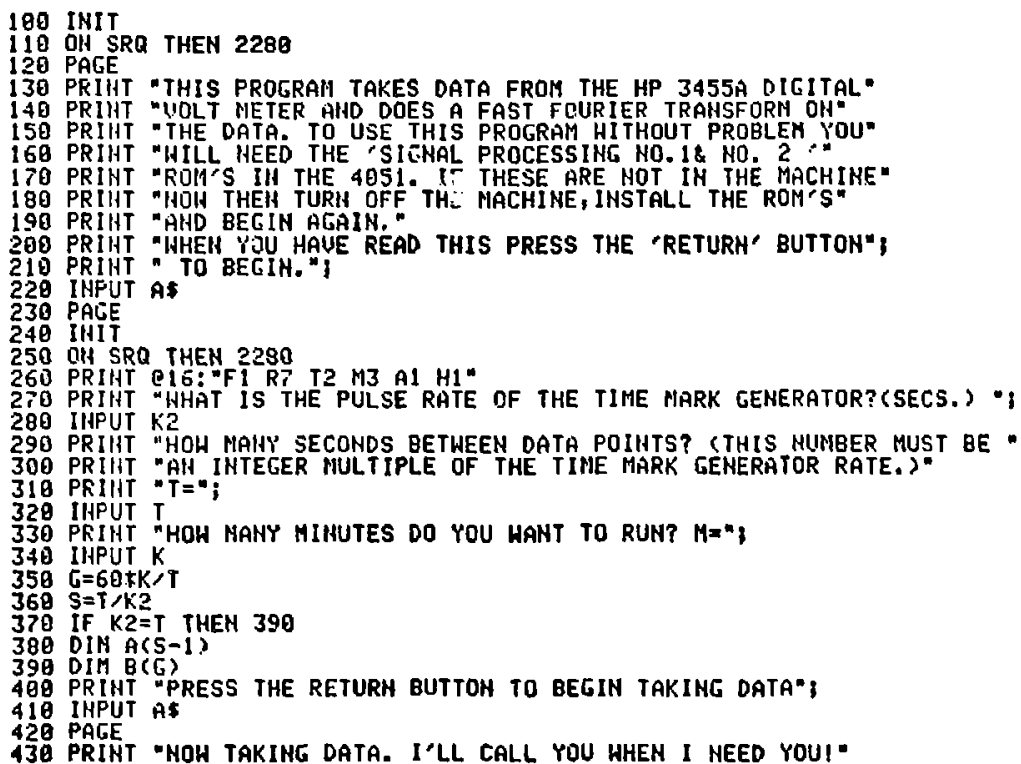


448 FOR $I=1$ TO $C$

450 IHPUT CI6:B(I)

468 IF $K 2=T$ THEN 480

470 INPUT EI6:A

489 HEXT I

498 OH EOF (O) THEH $59 \theta$

500 PRIAT "GGGGGG"

510 FRINT "DO YOU HAKT THIS DATA OH TAPE? (T OR N) *

520 IHPUT As

530 IF $A E=" Y "$ THEH 580

540 IF $A \$ \approx n$ " THEN 648

559 PRINT TY OR H OHLY PLEASE! *

560 IHPUT A\$

570 GO TO 530

580 PRINT "HHAT TAPE FILE HOULD YOU LIKE TO USE? F="3

590 IIPPUT $F$

603 PRIHT "IMSERT THE DATA TAPE AHD PRESS THE 'RETURH' KEY.";

610 IHPUT At

620 FIHD F

630 FRINT CJ3:B

64 PRIHT "DO YOU WAHT THE DATA DISPLAYED? (Y OR M) "

650 IHPUT $A+$

66 IF $A=" Y "$ THEN 690

670 IF $A S=" N "$ THEN 360

68 CO TO $6 \$ 0$

690 CALL "NIH", B,ML, II

300 CALL "NAX":B, M2, II

710 HINDON $1, G, M i, M 2$

720 UIEHPORT $20,100,20,80$

739 PALE

740 CALL "DISP",

750 PRIAT 1

760 PRINT "DO YOU HANT TO DO A FAST FOURIER TRANSFORH OH"

70 PRIHT " THIS DATA? (Y OR N) *

780 INPUT AF 
790 IF $A \$=" Y "$ THEN 930

BEO IF $A \$=" H "$ THEH 828

810 C0 T0 760

B26 REH

830 PRIHT "DO YOU HAHT TO RUN AGALTTCY OR M) "

840 INPUT AS

859 IF $A \$=-Y=$ THEN 230

860 IF AS= "U" THEH 930

870 FRIHT "Y OR $\mathrm{H}$ ONLY PLEASE! "I

880 IHPUT A

390 CO TO 850

900 PAGE

910 PR!HT "GGGGGG", "FINISHED, COODBYE"

929 Fito

930 DAGE

940 PRILI "DO YOU HAHT TO USE THE TAPER FUNCTION OH THE DATA?CY OR N) ";

950 INPUT As

960 IF $A F=" N "$ THEN 1118

978 IF $A \$=" Y "$ THEN 1910

989 PRINT "Y OR H ONLY PLEASE! -

990 IHPUT A

i०ด0 60 To 968

$1010 \mathrm{Y}=1$

1820 PRIINT -HOH MUCH TAPER HOULD YOU LIKE?(IN PERCENT FROM 10 TO 50) ";

1 B30 IliPUT PI

1040 IF PICIB OR P1)50 THEN 1060

1050 CO TO 1090

1069 PRINT "THIS NUMBER MUST BE BETHEEN 10 AND 501 !

1070 INPUT PI

1080 Go TO 1040

1090 PI $=P 1 / 109$

1108 Go TO 1120

$1110 \quad y=0$

1120 GO TO 1148
1230 PRINT "READ THE INSTRUCTINA AND TRY AGAIN" 
1140 PRIHT EENTER THE MUMBER OF POINTS"

1139 PRIAT "THIS NUTBER MUST BE 128,256,512, OR 1824 "1

1160 INPUT RI

i 170 R $R=R 1$

1180 IF $R I=128$ OR $R I=256$ OR $R I=512$ OR RI=1024 THEN 1210

119060 TO 1130

1200 DELETE $A$

1210 DIII $X(G)$

$1220 \mathrm{n}=\mathrm{B}$

1230 DELETE $B$

1240 DIH B(RI)

I250 PR!NT "HON MANY MICRONS PER DATA POINT? ";

1260 IIPUT $S$

1270 FOR $t=1$ TO RI

$1280 \mathrm{~B}(1)=\mathrm{K}(1)$

I29B NEXT

1300 DELETE $X$

1318 DIA $L(R 1 / 2+1), P(R 1 / 2+1)$

1320 CALL MIN, B, H1, I2

1330 CALL MMAX", B, H2,12

1340 FDR I $=1$ TO RI

$1350 B(1)=B(1)-(H I+N L) / 2$

1360 MEXT 1

1370 IF $Y=1$ THEN 1390

1380 IF $Y=0$ THEN 1420

1390 PACE

1400 PRINT "GES", "TAPERING THE DATA, BE BACK IN a MIMUTE"

1410 CALL "TAPER", B, PI

1420 PAGE

143 B PRINT "GGE", "DOING THE FFT, IT HILL TAKE A FEH MINUTES"

1440 CALL "FFT",

1430 CALL "POLAR", B,L,P

1460 CALL "MIN",L,ML,IL

1470 CALL - MAX', i,MZ.II

$1480 \quad Z=42$ 


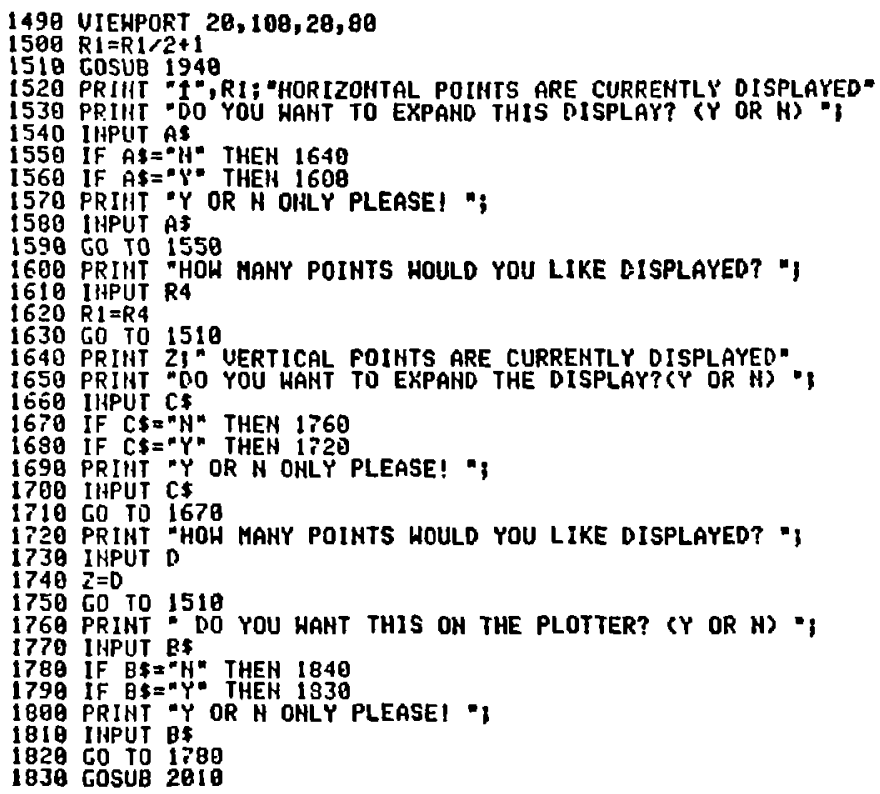




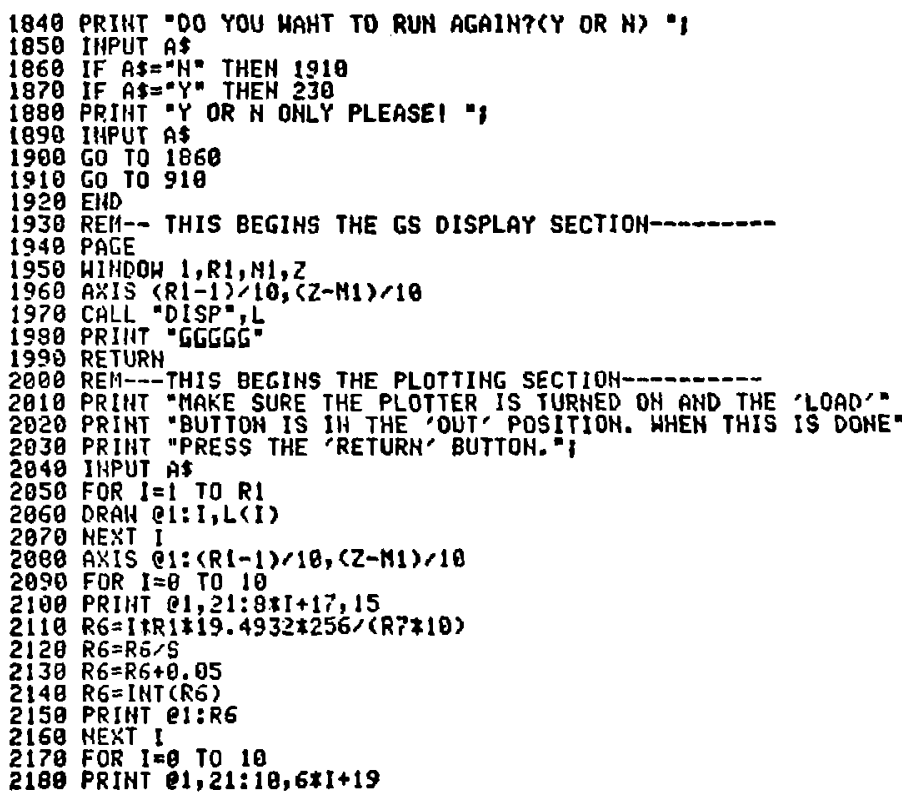




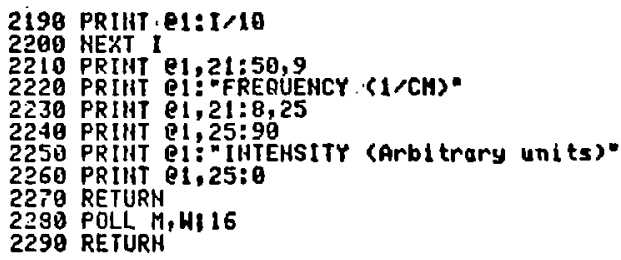




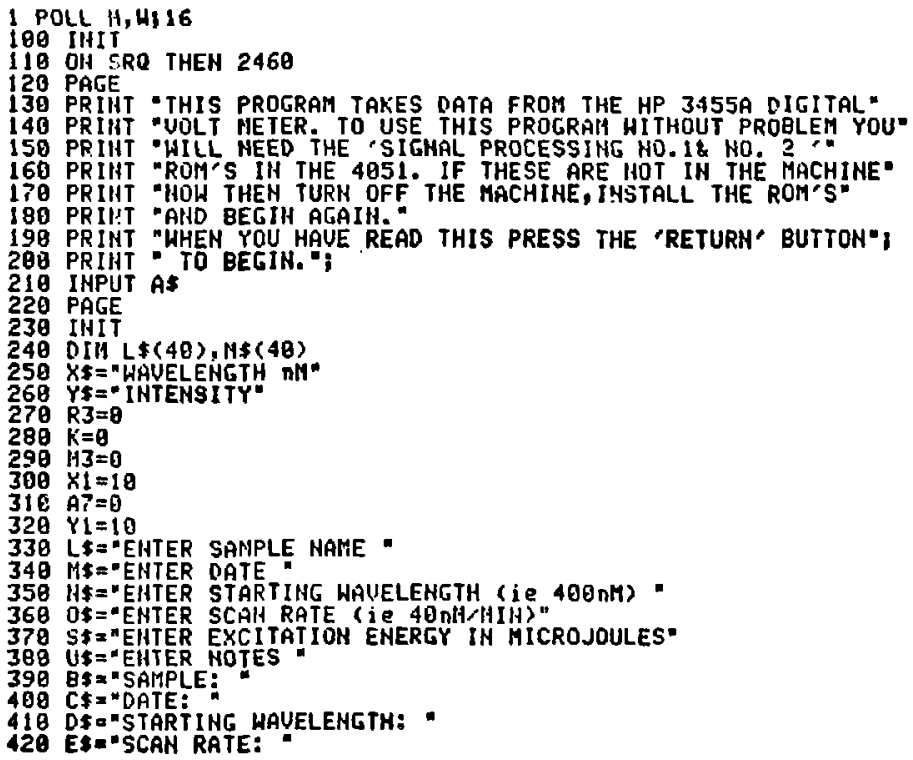




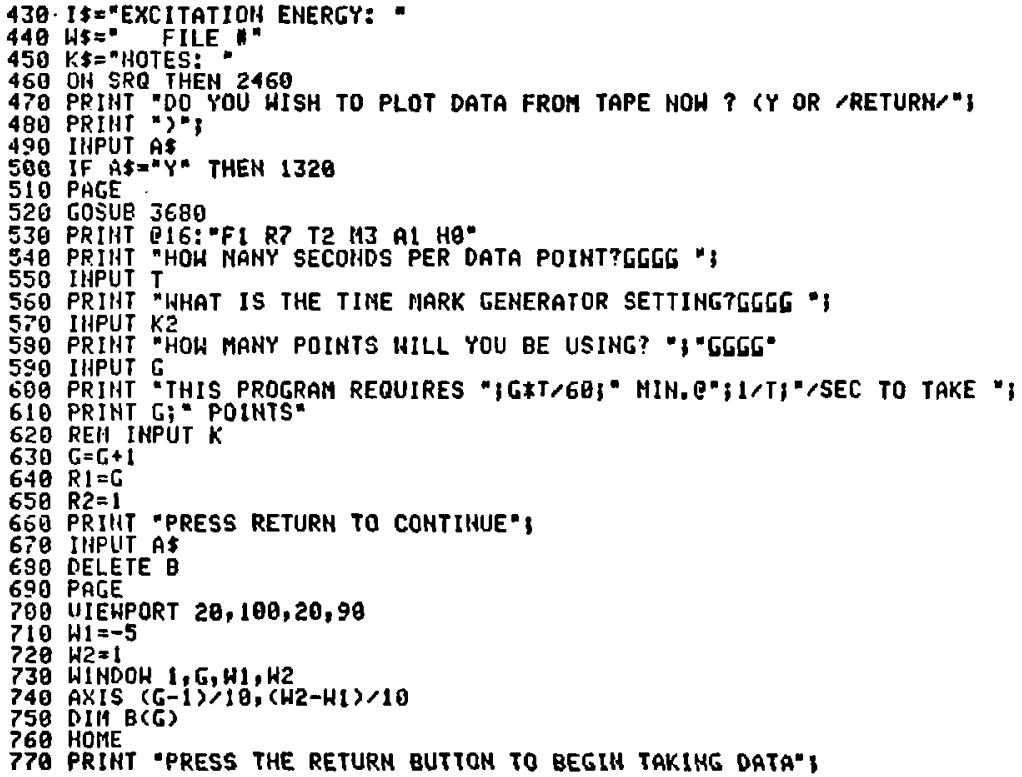




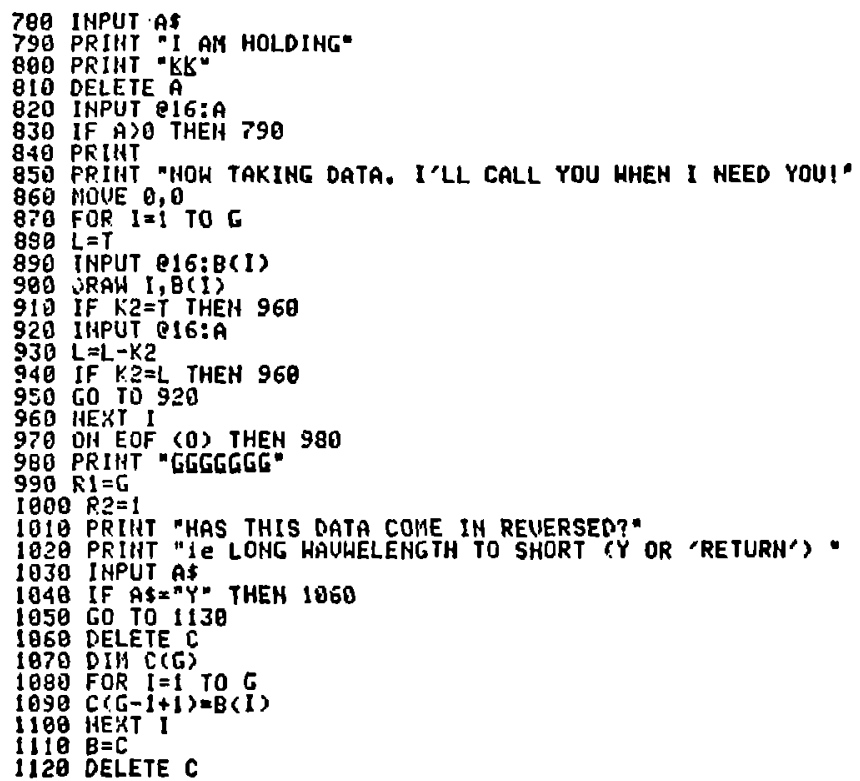




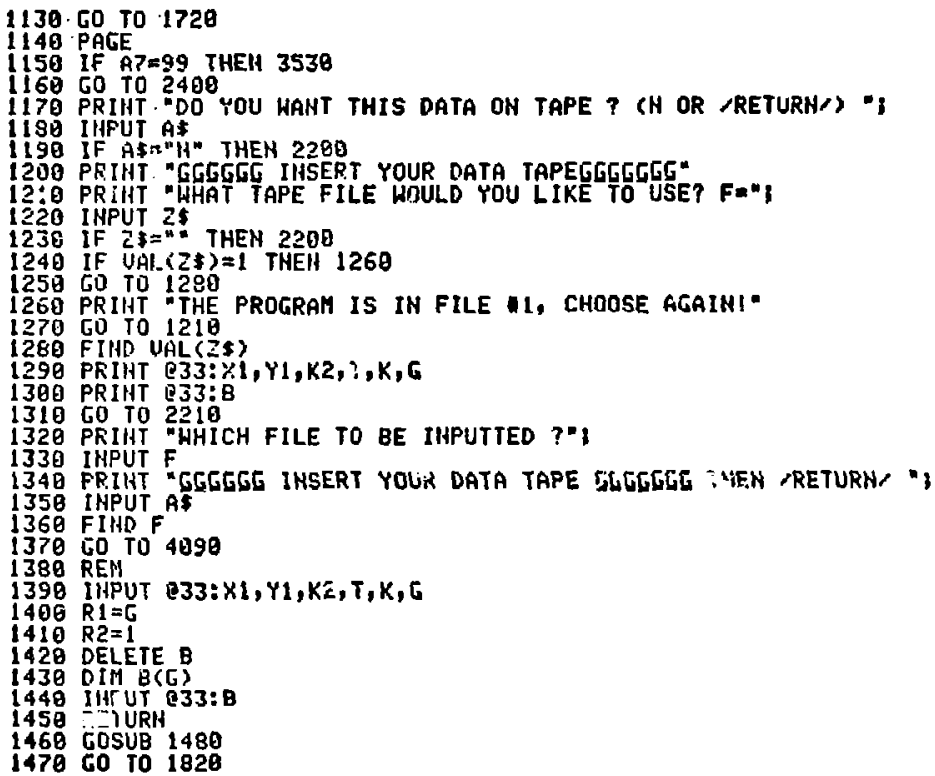




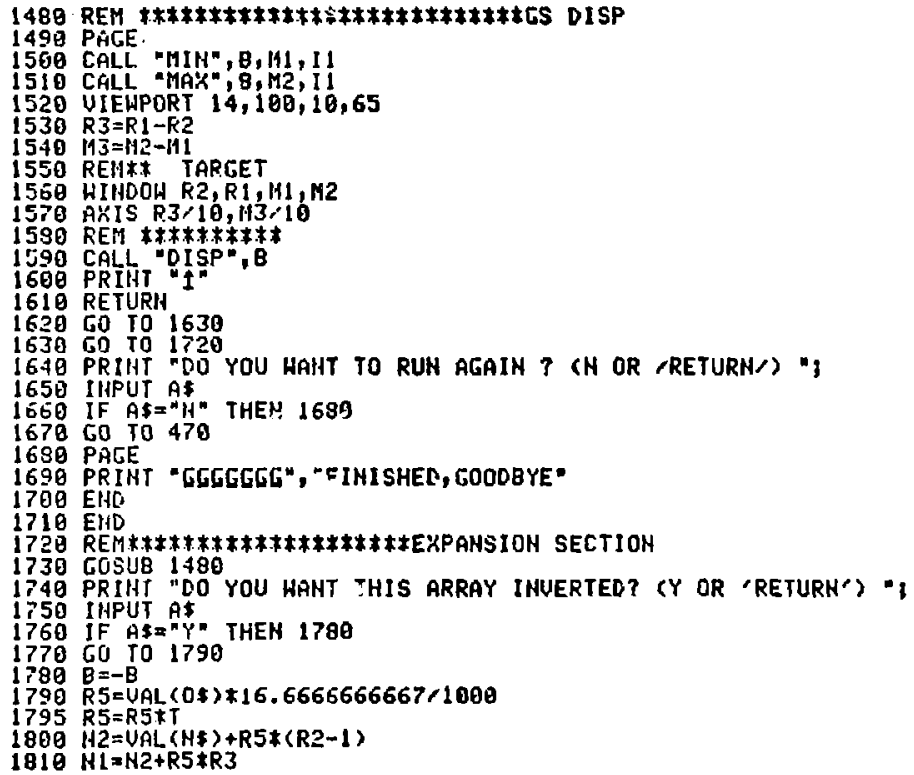




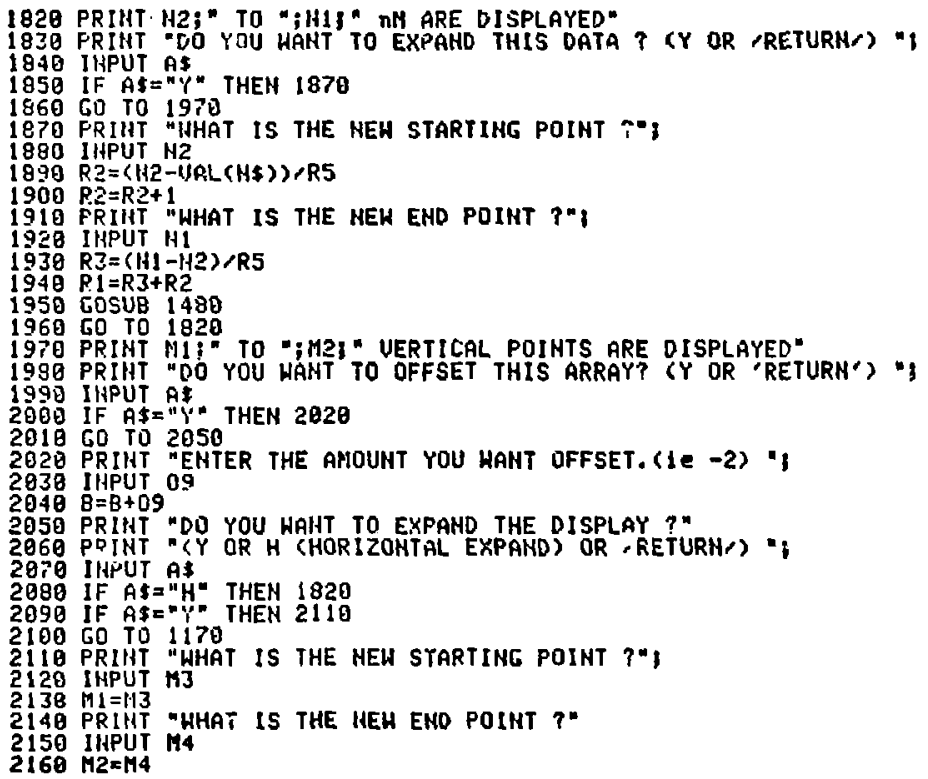




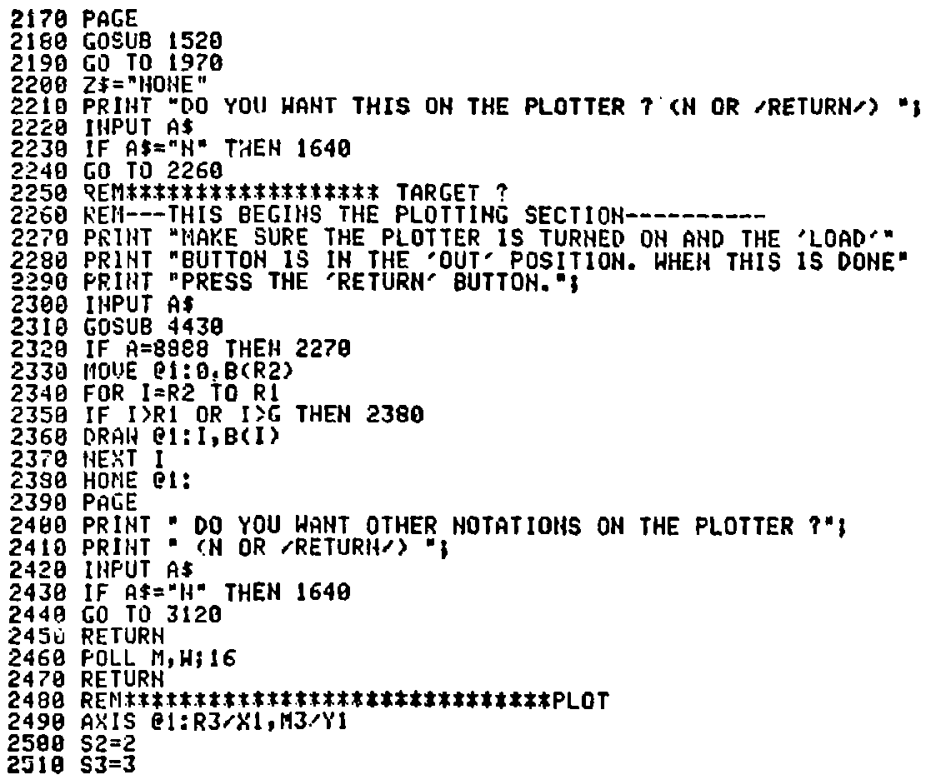




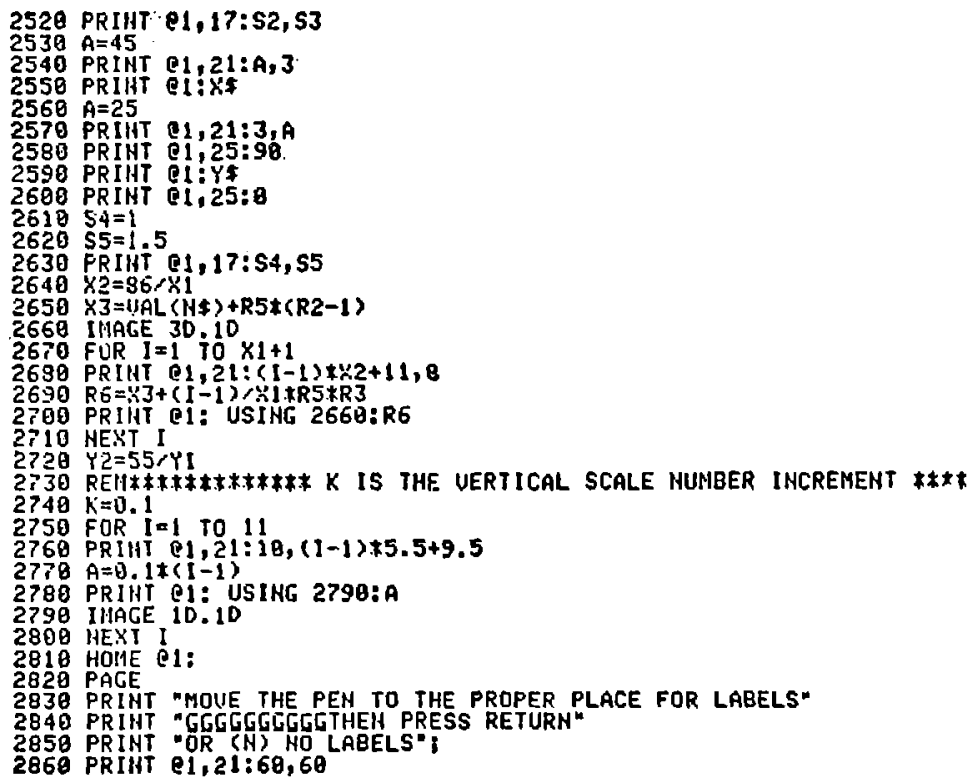




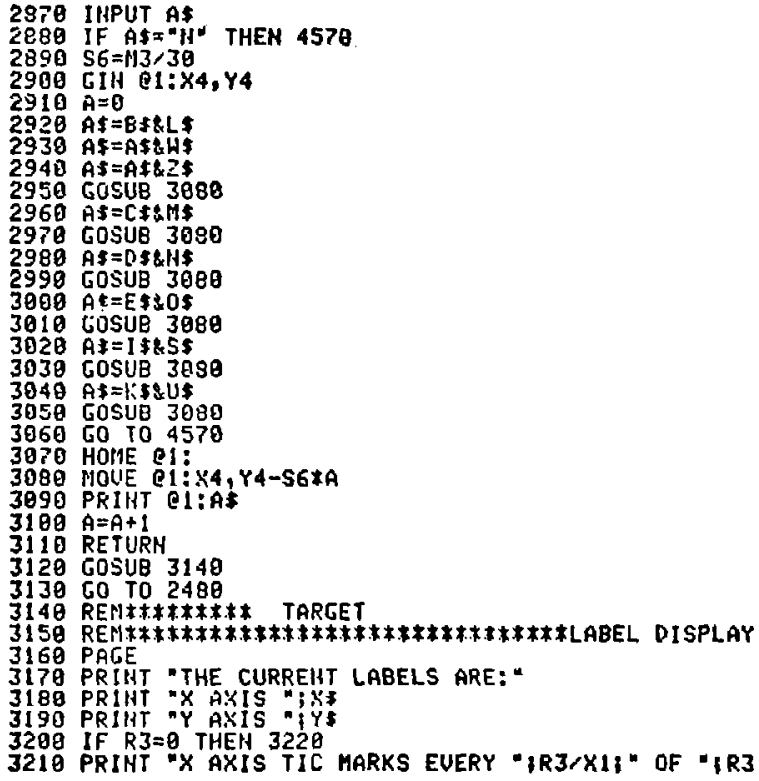




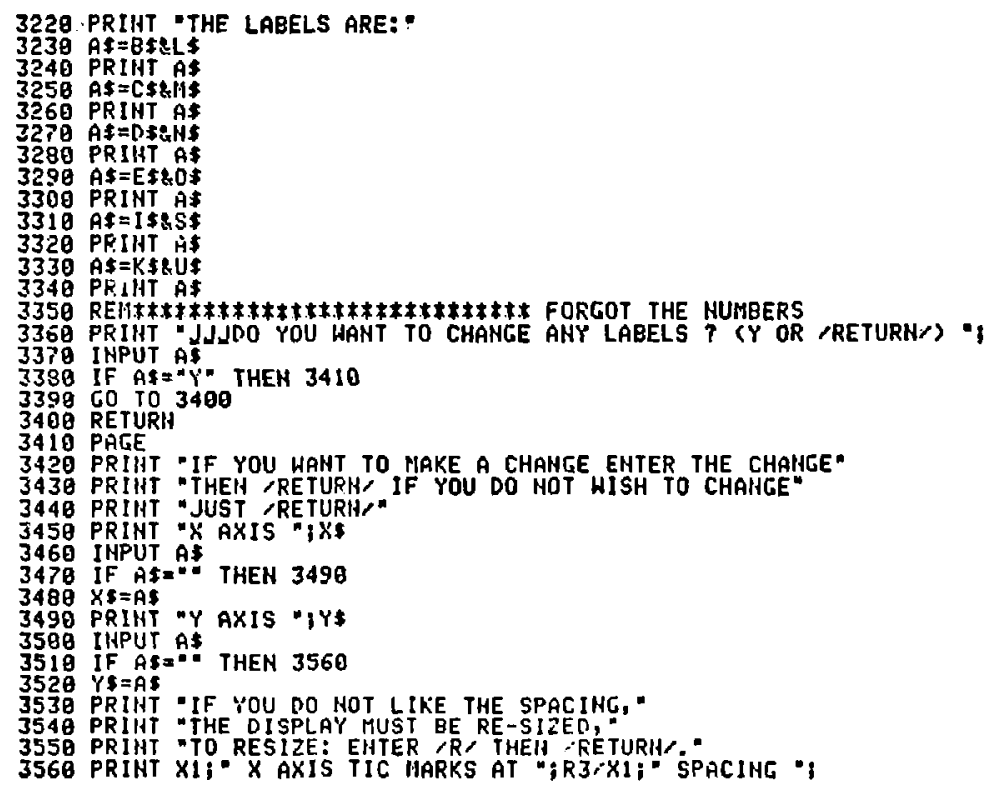




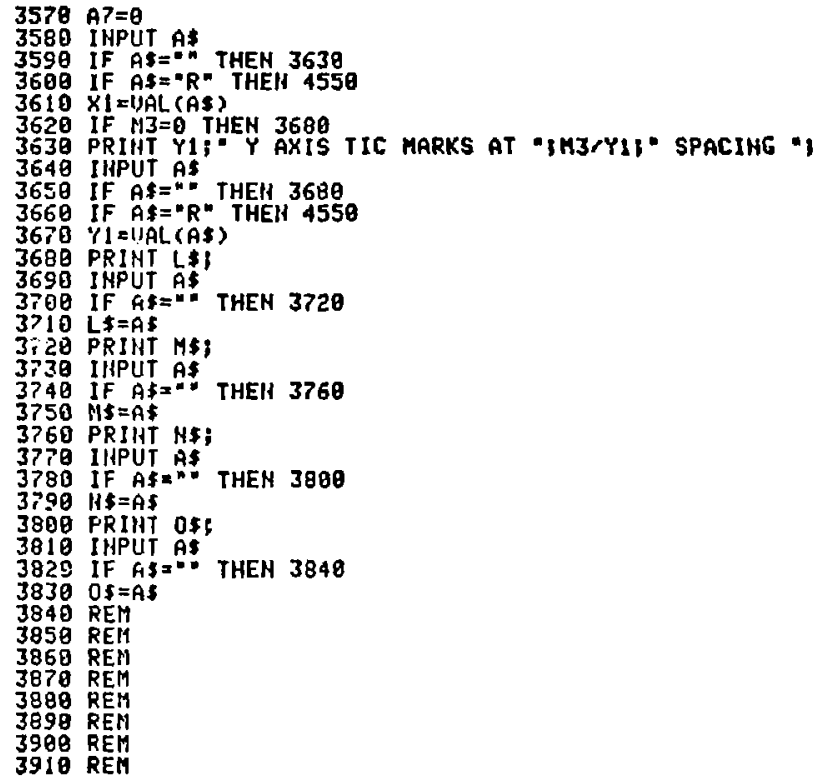




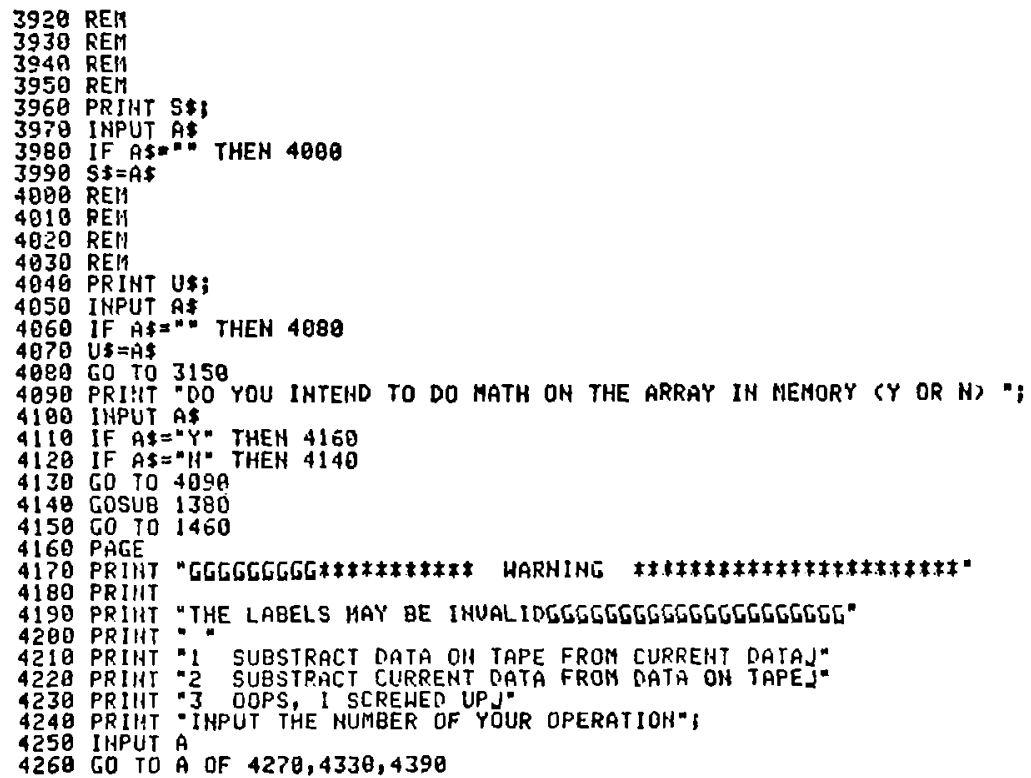


4270 DELETE C

4280 DIM C (G)

$4290 \mathrm{C}=\mathrm{B}$

$\$ 300$ GOSUB 1380

$4310 \quad B=C-B$

4320 CO TO 1468

4330 DELETE $C$

$\$ 3.40$ DIIA COG,

$4350 \quad C=B$

4350 GOSUB 1380

$4370 \quad \theta=B-C$

4399 60 TO 1469

4390 PRIVT = 9000 "

4400 PAGE

$4410 \mathrm{FOR}[=1$ TO 20

4420 PRIHT "GGGGGGGGG UNLOAD THE PRINTER GGGGGGGG"

4430 GIN $\mathrm{CI}_{1: \mathrm{A}, \mathrm{I}}$

4440 MOVE $C I: A+A>100,1+[/ 100$

4459 GIN CI:Al, I1

4460 IF $A=A I$ AHD I II THEH 4498

$4473 \quad \bar{H}=9999$

4420 RETURH

4490 PACE

4580 FOR I 1 TO 3

451日 PRIKT "GGGGEGGGEGGGGGGGG UHLOAD IT DUMMY GGGGGGGGGGGG"

4520 NEXT

$4530 \mathrm{~A}=8888$

4540 RETURH

4550 A7 $=99$

4560 GO TO 1138

4579 PRINT DO YOU HANT TO RE-GRAPH ? (Y OR IRETURH ) -I

4500 INPUT AS

$\$ 590$ IF $A \$=" Y$. THEN 1720

4600 CO TO 1640 